\title{
Wireless Sensor Network for Electric Transmission Line Monitoring
}

\author{
Final Technical Report
}

DOE Award: DE-FC26-06NT42795

January 2010

Revised March 2010

University of Louisville, Genscape Inc.

Principle Investigator: Bruce Alphenaar PhD

George Lin PhD, Bill Brown BE, Deirdre Alphenaar PhD, Walter Jones PhD, Chris Pettus ME, Yang Xu PhD, Devin Phinney BS 


\section{Contents}

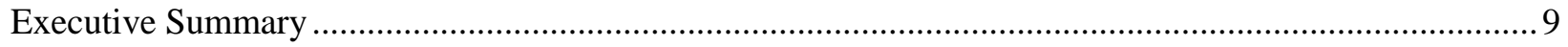

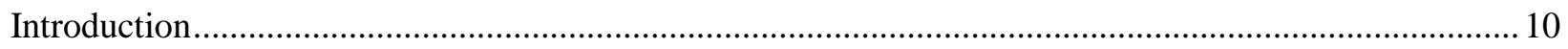

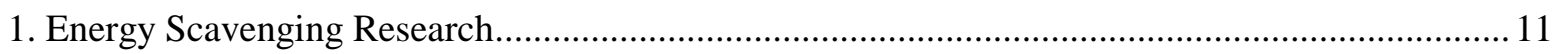

2. Research and Design of Methods for Utilizing Directional Sensors ........................................... 11

3. Prototype Development of Power Scavenging Devices, Directional Sensors ................................ 11

4. Integration Activities Including Form Factor / Function / Cost Optimization................................. 11

5. Embedded Application Development to Manage Monitor Power, Data Acquisition, Processing

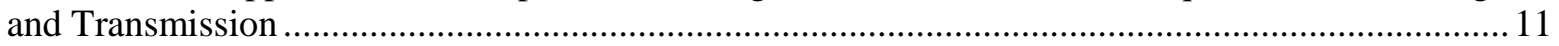

6. Field Test and Deployment of Novel Sensors and Power Sources ............................................. 12

7. Server Side Application Development.................................................................................... 12

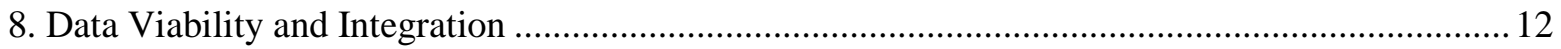

1 Alternative Power Sources / Power Scavenging Research …......................................................... 12

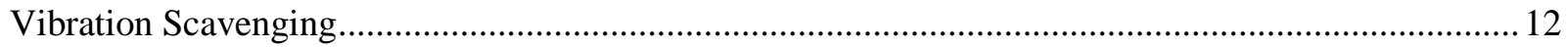

Theoretical Modeling of Magnetically Coupled Cantilever Designs................................................... 20

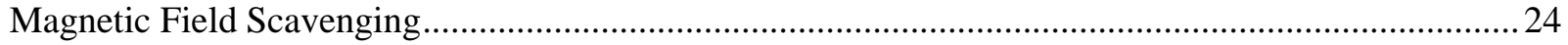

2 Research and Design of Methods for Utilizing Directional Sensors ..............................................25

2.1 Multiple Line Sensing - Tri-axial (or directional) magnetic field sensors ................................25

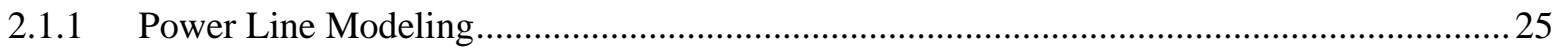

2.1.2 Directional Sensing Techniques.................................................................................. 28

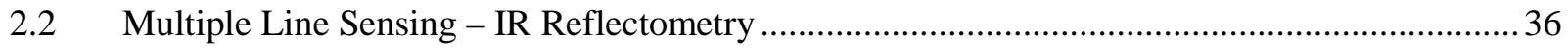

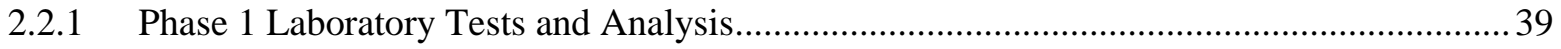

2.2.2 Phase 1 Power Line Field Tests and Analysis................................................................ 40

2.2.3 Phase 2 Reflectometer Signal-to-Noise Optimization.......................................................... 43

3 Prototype Development of Power Scavengers and Directional Sensors.......................................... 44

3.1 Prototype Development of Power Scavengers ........................................................................ 44

Vibration Scavenging - Prototype Design and Field Testing ...........................................................4 44

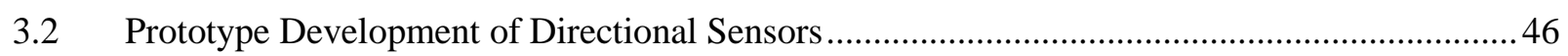

3.2.1 Prototype and Commercial Calibration Systems............................................................... 46

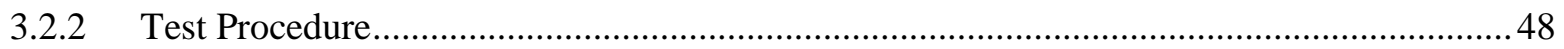

3.2.3 Oakridge DC Powerline Test Facility …........................................................................ 49

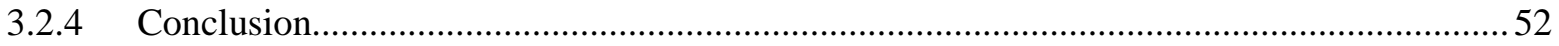

4 Integration Activities Including Form Factor / Function / Cost Optimization..................................... 53

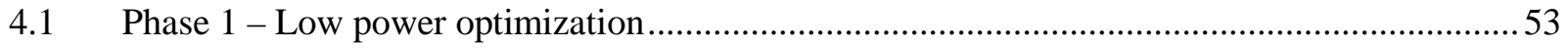


4.1.1 Processor implementation using low power Crossbow Mote platform............................... 54

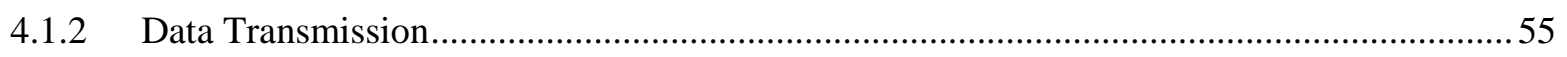

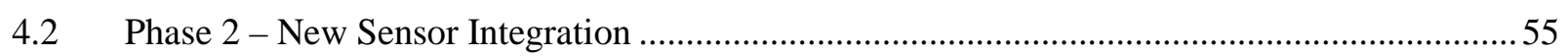

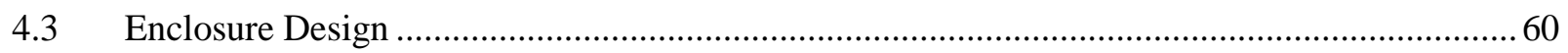

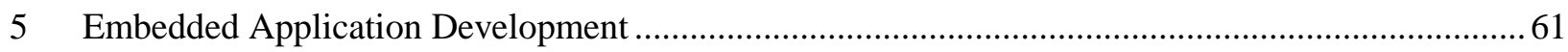

$6 \quad$ Field Test and Deployment of Novel Sensors and Power Sources ................................................ 63

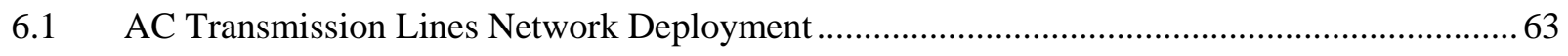

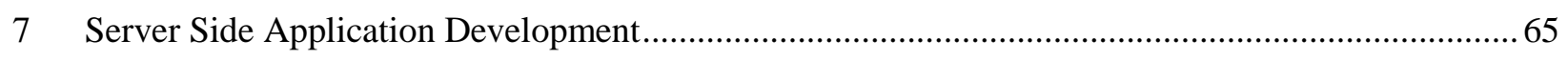

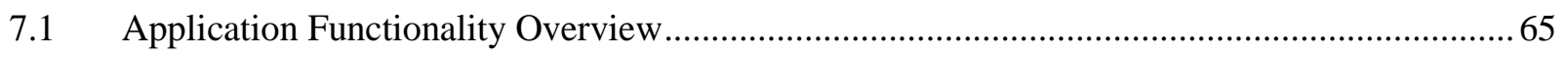

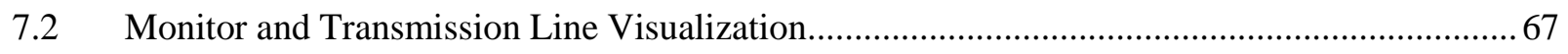

7.3 Transmission Line Alert and Alert Visualization Application ............................................... 68

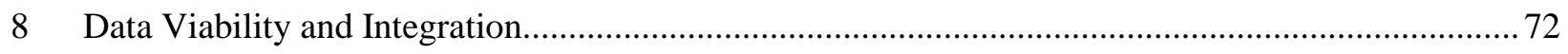

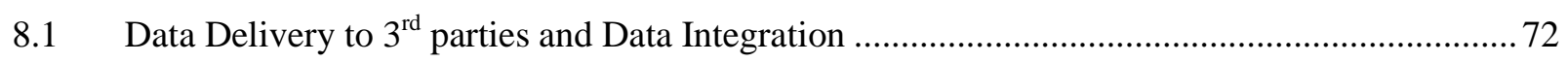

9 Cost Analysis for Wireless Transmission Line Networks ............................................................... 75

Appendix A

\section{Tables}

Table 1-1: Vibration measurements close to a busy urban street............................................................. 13

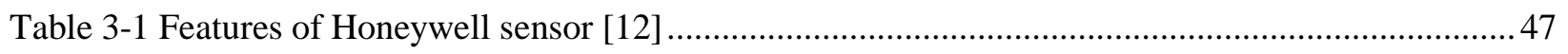

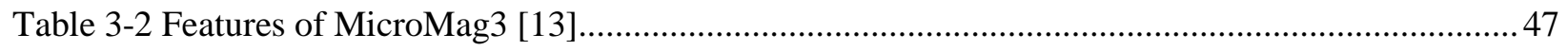

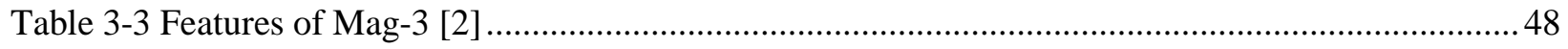

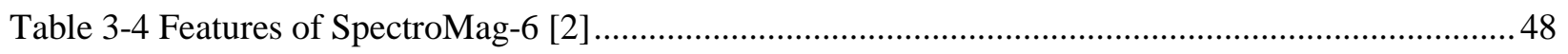

Table 4-1: Proposed power requirements and sources for transmission line monitor ............................... 53

\section{Figures}

Figure 1-1: Simple Cantilever Design and Voltage Harvesting Circuit................................................ 13

Figure 1-2: Sample schema to achieve voltage amplification and optimal frequency response using a magnetically coupled cantilever scheme for energy harvesting.

Figure 1-3: Sample voltage amplification as a function of typical environmental outdoor frequencies for an optimal magnetically coupled cantilever scheme such as that shown in Figure 1.2.......................... 15

Figure 1-4: (a) Set-up with fixed opposing magnet (b) Measurement circuit (c) Set-up with opposing

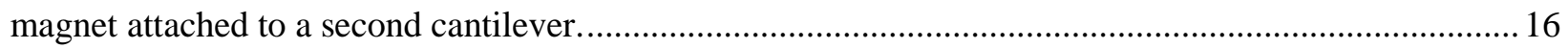

Figure 1-5: Voltage output of the piezoelectric cantilever as a function of shaker table frequency for (a) single cantilever (b) double cantilever. Integrated voltage output as a function of frequency for (c) single cantilever and (d) double cantilever.............................................................................................. 17

Figure 1-6: Non-rectified voltage output at $14 \mathrm{~Hz}$ vibration for coupled (solid line) and uncoupled

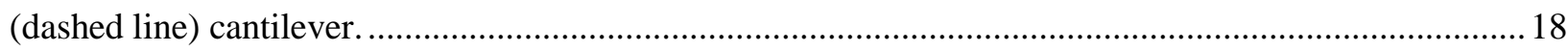


Figure 1-7: (a) The schematic of the storage circuit, and (b) DC voltage output measured on the storage capacitor indicating more charge is stored with the magnetic coupling setup........................................ 19

Figure 1-8: Integrated voltage output versus vibration acceleration magnitude for three different diameter magnets. In each case, the coupled (blue line) is compared to the uncoupled (red line)......................... 20

Figure 1-9: Model of the strained piezoelectric cantilever ................................................................ 21

Figure 1-10: (a) Modified one-dimensional spring force model. A is the source acceleration, $k$ is the spring constant, $m$ is the proof mass, $z(t)$ is the cantilever deflection and $F_{B}(\eta, z)$ is the magnetic force.

(b) Apparatus used to measure the magnetic force $F_{B}(\eta, z)$.

Figure 1-11: (a) The magnetic force $F_{B}(\eta, z)$ for three separation distances $(\eta=4 \mathrm{~mm}, 5 \mathrm{~mm}$ and $10 \mathrm{~mm})$. The lines show fits to Equation 1.4. Also shown (as a straight line) is the spring force of the cantilever. (b) The cantilever spring potential (dashed line) and the potential due to the combination of the restoring force and the magnetic force for the 3 separation distances. ................................................................2 22

Figure 1-12: Simulated output of the piezoelectric cantilever for the case of no magnetic coupling (dashed line) and magnetic coupling (solid line).

Figure 1-13: Voltage doubling circuit to maximize voltage output from induced voltage on the pick-up solenoid in the presence of a typical magnetic field measured under a high voltage transmission line. .... 24 Figure 2-1: a. Line configuration example for two transmission lines separated by 60 feet. Sensor is placed 45 feet from left line and 15 feet from the right line. b. The resultant magnetic field vector for three different power flow distributions (power flow on right line only, power flow on left line only and equal power flow on both lines).

Figure 2-2: a. Circuit design for simulated power line electromagnetic field testing in the laboratory. $b$. Line configurations and power distributions tested using the laboratory simulation.....

Figure 2-3: Comparison between the angle of vector rotation under the model line (Bz_2 mG) and 4cm from the model line $\left(\mathrm{Bz} \_1 \mathrm{mG}\right)$ 28

Figure 2-4 a. Parallel, b. perpendicular and c. crossed transmission lines. The indicated monitor locations were tested as solutions to provide a method of measuring power flow on each line independently......... 30 Figure 2-5: Schematic drawing of two monitors symmetrically placed with respect to the centerline of the

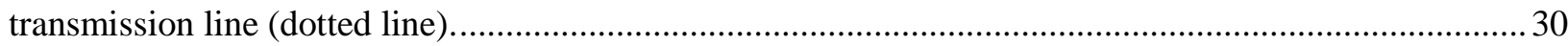
Figure 2-6: Sample magnetic field measurement by two monitors (1, 2). Five $60 \mathrm{~Hz}$ cycles (amplitude in arbitrary analog-to-digital conversion units) are shown. 32

Figure 2-7: Computed values for Equations 2.5, 2.6, 2.7 using actual field measurements for a high voltage overhead transmission line. The computed cancellation is non-zero due to variations in measured values from the theoretically computed values (milliGauss).................................................................33

Figure 2-8: Schematic drawing of signal cancellation for parallel horizontal lines ................................. 34 Figure 2-9: Magnetic field measurement by two monitors placed symmetric to line B (Figure 2-8) for a horizontal parallel line set. (Signal amplitude in arbitrary analog-to-digital conversion units).................. 35

Figure 2-10: The blue line represents the total acquired signal after cancellation. The red line represents the remaining signal representing Line A power flow (arbitrary relative signal values).......................... 36

Figure 2-11: Vibro-optical detection scheme......................................................................................... 37

Figure 2-12: Power detection scheme based on Kerr rotation. Faraday rotation describes a similar effect for light transmitted (rather than reflected) through the magnetic medium............................................ 38 Figure 2-13 DFT spectrum (arbitrary units) acquired from shaker table set to vibrate at $60 \mathrm{~Hz}$. Significant

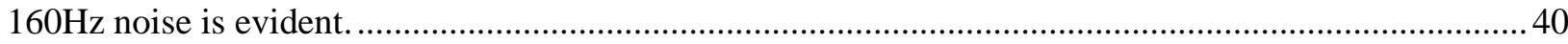
Figure 2-14 FFT spectrum (arbitrary units) acquired from fixed targets (laboratory door and wall)........ 40 
Figure 2-15 Reflectometer measurement positions relative to two parallel lines ( $345 \mathrm{kV}$ and . 41 Figure 2-16 The plots are arranged in the order in which data was acquired. Top Left: measurements of the front line with no current Top Right: measurements of the rear line (taken at position 1), Central Left: control measurements from wooden mounting pole with polarizer installed Central Right: measurements of the rear line (taken at position 2) Bottom Left: repeat measurement of the rear line (taken at position 2) Bottom Right: control measurements from wooden mounting pole with no polarizer installed.....

Figure 2-17 Power spectrum (arbitrary units) of the light amplitude reflected from a shaker table vibrating at $40 \mathrm{~Hz}$ using optimized reflectometer device.

Figure 3-1 Schematic showing simultaneous data acquisition of vibrations (using an accelerometer) and voltage output (from the vibration scavenging prototype) from a light pole on the bridge. Data acquisition is performed using a portable battery powered data logging device which stores data on a flash card interface for download to a PC for data analysis.

Figure 3-2 Photo showing the vibration scavenging prototype. The device is mounted on a flexible strap for mounting to a pole.

Figure 3-3 Voltage output from prototype scavenging device attached to a streetlight pole on a busy urban bridge. Output levels depend on bridge traffic with peaks associated with the passing of large vehicles such as trucks.

Figure 3-4 Sensor alignment with respect to the line was achieved with a wooden mounting structure in combination with the survey level.

Figure 3-5 ORNL DC Test Site ....

Figure 3-6 Controlled DC line current ramp on and ramp off transitions during testing......................... 50

Figure 3-7 Comparison of three commercial 3-axis sensors (Mag-03, Micro-Mag, Honeywell)..............50

Figure 3-8 Theoretical and actual magnetic field strengths with a 1000A current flow. Bx is the horizontal component of the magnetic field, By is the vertical component of the magnetic field, blue cross is $\mathrm{Bx}$ at a position 9 feet from the line, pink cross is By at a position 9 feet from the line, blue $\mathrm{X}$ is $\mathrm{Bx}$ at a position 35 feet from the line, pink $\mathrm{X}$ is $\mathrm{By}$ at a position 35 feet from the line.

Figure 3-9 Theoretical and actual magnetic field strengths with a 2000A current flow. Bx is the horizontal component of the magnetic field, By is the vertical component of the magnetic field, blue cross is $\mathrm{Bx}$ at a position 9 feet from the line, pink cross is By at a position 9 feet from the line, blue $\mathrm{X}$ is $\mathrm{Bx}$ at a position 35 feet from the line, pink $\mathrm{X}$ is $\mathrm{By}$ at a position 35 feet from the line......................................52

Figure 4-1 Current and proposed battery size and weight comparison................................................54

Figure 4-2 3-axis chip (mounted on breadboard) shown with two solenoid-based magnetometers (enclosed in black casing) 56

Figure 4-3 Graphical user interface to view and analyze the data acquired by the Mote processor from the 3-axis magnetic field sensor. .56

Figure 4-4 Development pathways corresponding to Phase 1 and Phase 2 prototypes. Items in color show the components being replaced and tested in each prototype stage. Prototype 1 has a replacement Mote processor, (Sensor, power, and communication modules remain the same as original). Prototype 2 adds a digital modem and integrates this with the Mote processor (Sensor and power modules remain the same as the reference system). Prototype 3 adds a 3-axis magnetometer and associated interface electronics and integrates these with the Mote processor (Power and communication modules remain the same as the reference system). 
Figure 4-5 Prototype 3 showing the 3-axis magnetometer sensor board integrated into a monitor with a

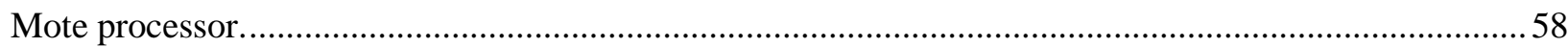

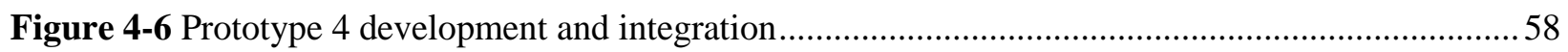

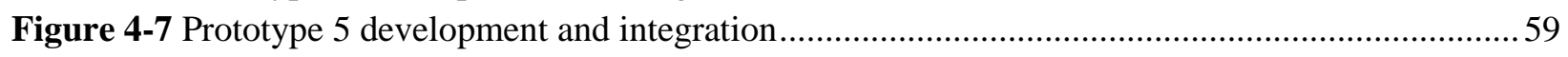

Figure 4-8 Side by side comparison of current integrated system board (left) to containing processor, 3axis magnetometer and power supply compared with original monitor (right) containing two separate processor and sensor boards and $12 \mathrm{~V}$ battery.

Figure 5-1 Magnetic field data acquired by the existing transmission line monitor (purple) and the prototype Mote-based transmission line monitor (blue) in a side-by-side deployment of both monitors at the transmission line test site shown in Figure 6.2.

Figure 5-2 Side-by-side deployment of existing transmission line monitor (right) and a prototype Motebased transmission line prototype (left) at a power line field test site.................................................. 62

Figure 6-1 Mid-Atlantic Area Strategic Transmission Corridor ........................................................... 64

Figure 6-2 Mote prototype transmission line monitor compared with standard calibration monitor ........ 64 Figure 6-3 Transmission line data from the prototype monitors for Massena-Marcy line outage event, October 18, 2008. .65

Figure 6-4 Transmission line data for Louden-Doubs line from the prototype monitor for line outage event, October 27, 2008.

Figure 7-1 Application overview for processing of transmission line alerts and transmission of line alerts to third parties.

Figure 7-2 Google Earth image showing transmission line monitors (square icons) geolocated with transmission line data. Available line data (alerts, name, capacity voltage etc) can be presented through

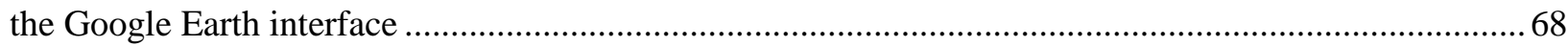

Figure 7-3 Data from a three hour window covering the September 18 event with no filtering applied... 70 Figure 7-4 Post-filtered view of the September $18^{\text {th }}$ event showing a concentration of events passing the

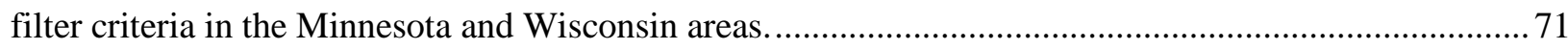

Figure 7-5 Google Earth visualization of transmission line events (red lines) associated with a lightening strike during tornado activity in the region shown............................................................................. 72

Figure 8-1 Main menu screen on transmission line kml file server application. .................................... 73

Figure 8-2 Transmission line outage signal detected using grid frequency monitoring network .............. 74 Figure 8-3 Frequency information from a transmission line outage event displayed on the Google Earth application. Red arrows show units detecting under-frequency signals. Green arrows show units detecting

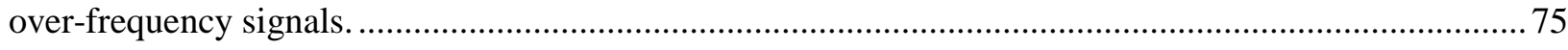

Figure 9-1 Distribution of costs associated with internal components of the wireless electric transmission line monitor. 76 


\section{DISCLAIMER}

This report was prepared as an account of work sponsored by an agency of the United States Government. Neither the United States Government nor any agency thereof, nor any of their employees, makes any warranty, express or implied, or assumes any legal liability or responsibility for the accuracy, completeness, or usefulness of any information, apparatus, product, or process disclosed, or represents that its use would not infringe privately owned rights. Reference herein to any specific commercial product, process, or service by trade name, trademark, manufacturer, or otherwise does not necessarily constitute or imply its endorsement, recommendation, or favoring by the United States Government or any agency thereof. The views and opinions of authors expressed herein do not necessarily state or reflect those of the United States Government or any agency thereof. 


\section{Executive Summary}

Generally, federal agencies tasked to oversee power grid reliability are dependent on data from grid infrastructure owners and operators in order to obtain a basic level of situational awareness. Since there are many owners and operators involved in the day-to-day functioning of the power grid, the task of accessing, aggregating and analyzing grid information from these sources is not a trivial one. Seemingly basic tasks such as synchronizing data timestamps between many different data providers and sources can be difficult as evidenced during the post-event analysis of the August 2003 blackout ${ }^{1}$.

In this project we investigate the efficacy and cost effectiveness of deploying a network of wireless power line monitoring devices as a method of independently monitoring key parts of the power grid as a complement to the data which is currently available to federal agencies from grid system operators. Such a network is modeled on proprietary power line monitoring technologies and networks invented, developed and deployed by Genscape, a Louisville, Kentucky based realtime energy information provider ${ }^{2}$. Genscape measures transmission line power flow using measurements of electromagnetic fields under overhead high voltage transmission power lines in the United States and Europe.

Opportunities for optimization of the commercial power line monitoring technology were investigated in this project to enable lower power consumption, lower cost and improvements to measurement methodologies. These optimizations were performed in order to better enable the use of wireless transmission line monitors in large network deployments (perhaps covering several thousand power lines) for federal situational awareness needs.

Power consumption and cost reduction were addressed by developing a power line monitor using a low power, low cost wireless telemetry platform known as the "Mote" ${ }^{3,4}$, Motes were first developed as smart sensor nodes in wireless mesh networking applications. On such a platform, it has been demonstrated in this project that wireless monitoring units can effectively deliver real-time transmission line power flow information for less than $\$ 500$ per monitor. The data delivered by such a monitor has during the course of the project been integrated with a national grid situational awareness visualization platform developed by Oak Ridge National Laboratory ${ }^{5}$.

Novel vibration energy scavenging methods based on piezoelectric cantilevers were also developed as a proposed method to power such monitors, with a goal of further cost reduction and large-scale deployment. Scavenging methods developed during the project resulted in 50\% greater power output than conventional cantilever-based vibrational energy scavenging devices ${ }^{8,9}$ typically used to power smart sensor nodes. 
Lastly, enhanced and new methods for electromagnetic field sensing using multi-axis magnetometers and infrared reflectometry were investigated for potential monitoring applications in situations with a high density of power lines or high levels of background $60 \mathrm{~Hz}$ noise in order to isolate power lines of interest from other power lines in close proximity.

Introduction

The goal of this project was to investigate and demonstrate the feasibility of using small form factor, highly optimized, low cost, low power, non-contact, wireless electric transmission line monitors for delivery of real-time, independent power line monitoring for the US power grid.

The project was divided into three main types of activity as follows;

- Research into expanding the range of applications for non-contact power line monitoring to enable large scale low cost sensor network deployments (Tasks 1, 2)

- Optimization of individual sensor hardware components to reduce size, cost and power consumption and testing in a pilot field study (Tasks 3,5)

- Demonstration of the feasibility of using the data from the network of power line monitors via a range of custom developed alerting and data visualization applications to deliver real-time information to federal agencies and others tasked with grid reliability (Tasks 6,8) 


\section{Project Tasks:}

1. Energy Scavenging Research - Power sources for wireless devices generally contribute to increasing the size and cost of the wireless sensor system. To enable the mass deployment of large numbers of wireless sensors for electric transmission line monitoring, vibration energy scavenging was investigated as a means of powering these devices.

2. Research and Design of Methods for Utilizing Directional Sensors - Directional sensors were investigated to spatially isolate the electromagnetic field from a particular power line where two or more power lines are in close proximity (referred to in this project as a "high line density" situation). These occurrences are most common in urban or industrial settings. A three-axis magnetic field sensor was integrated within the power line monitor and tested on AC and DC transmission power lines. In addition, a novel method of measuring current in overhead transmission lines was investigated using a mobile handheld infrared reflectometry technique.

3. Prototype Development of Power Scavenging Devices, Directional Sensors and Monitoring Device - Vibration scavenging devices and associated prototype electronic interface boards were designed and developed. Power conversion device designs were tested in the laboratory and in the field to establish optimal designs. Various multi-directional magnetic field sensors were developed and tested. Following design of an optimal directional sensor array in the laboratory, a testing platform and data acquisition system was designed for field testing.

4. Integration Activities Including Form Factor / Function / Cost Optimization - a wireless power line monitor incorporating single axis and multi-axis magnetic field sensors, low power processor technology (Crossbow Mote TelosB Mote Research Platform (TPR2400CA)), optimized communication technology and small form factor was integrated into a single power line monitoring device. The unit was field tested side-by-side with a commercial power line monitoring system for comparison and calibration purposes.

\section{Embedded Application Development to Manage Monitor Power, Data Acquisition,} Processing and Transmission - Embedded firmware applications on the Mote platform were developed using the open-source TinyOS operating system. Data acquisition, on-board processing and data transmission protocols in TinyOS were optimized for low power consumption during operation. 
6. Field Test and Deployment of Novel Sensors and Power Sources - A small network of prototype power line monitors were deployed and tested over a six to nine month period on key congested transmission lines on the US grid. The deployed network provided a proof-of-concept for potential applications of the wireless transmission line monitor for federal power grid situational awareness. A cost analysis was performed to allow insight into associated costs with the deployment of such a system.

7. Server Side Application Development Software applications were developed to enable data reception, data storage, data processing, geospatial event processing and event alert delivery using data from the power line monitoring network. These applications enable data delivery and integration into $3^{\text {rd }}$ party data visualization and analysis systems.

8. Data Viability and Integration - A custom Google Earth based web service application was developed to deliver power line data and geospatial grid event alerts. The application was provided to Oak Ridge National Laboratory (ORNL) for testing and review during the course of the project. Proof-of-concept integration with ORNL's national power line situational awareness platform or Visualizing Energy Resources Dynamically on the Earth (VERDE) system which is a Google Earth based platform was performed. The VERDE platform is currently used as an active situational awareness tool by the Infrastructure Security and Energy Restoration (ISER) Division of the U.S. Department of Energy's Office of Electricity Delivery and Energy Reliability (OE). The goal of this task was to allow DOE to determine the ease of use of data from a wireless electric transmission line monitoring network.

\section{Alternative Power Sources / Power Scavenging Research}

\section{Vibration Scavenging}

Inspired by the work of Roundy, Wright and Rabaey ${ }^{6,7}$ (where vibrational energy scavenging was used to power low power Mote processors such as those used here for the wireless electric transmission line monitor platform), various methods to optimize the power output capable from energy scavenging were investigated. In the Roundy, Wright and Rabaey study, sufficient power could be scavenged from footsteps resounding on a wooden deck indoors to allow the Mote device sufficient energy to make a data transmission every 50 minutes. Outdoor vibrations are typically characterized by low amplitude and a broadband spectrum without distinct resonant frequency peaks. Table 1-1 shows some typical vibrations measured in an urban street setting. 
Table 1-1: Vibration measurements close to a busy urban street

\begin{tabular}{|c|c|c|c|}
\hline SOURCE & $\begin{array}{c}\text { FREQUENCY } \\
(\mathrm{Hz})\end{array}$ & $\begin{array}{c}\text { AMPLITUDE } \\
(\mathrm{m} / \mathrm{s} 2)\end{array}$ & $\begin{array}{c}\text { POWER OUTPUT } \\
(\mathrm{mW})\end{array}$ \\
\hline Footsteps on a wooden deck $^{1}$ & 385 & 1.3 & 45 (every 50 mins) \\
\hline Pavement Flagstone $^{\#}$ & $10-30$ & 0.5 & \\
\hline Steel pole cemented into pavement $^{\#}$ & $10-20$ & 0.06 & \\
\hline
\end{tabular}

\# Measured using seismic accelerometer on a street during busy traffic period. Frequency denotes typical frequency ranges.
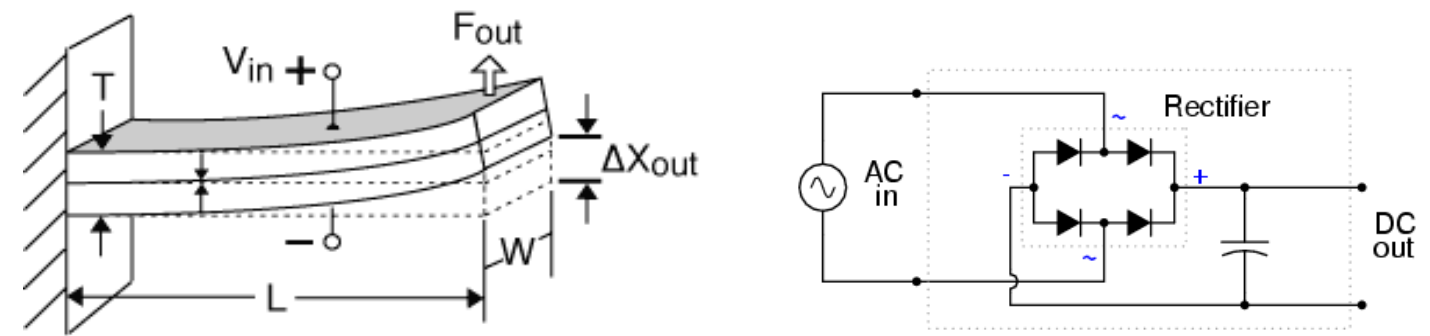

Figure 1-1: Simple Cantilever Design and Voltage Harvesting Circuit

A magnetically coupled scheme was devised to optimize the use of piezoelectric PZT (Lead Zirconate Titanate) cantilevers (Figure 1-1) for use in outdoor vibration energy scavenging applications. The objective was to achieve a vibration scavenging device design that satisfied the following requirements;

- Ability to tune to variable environmental frequency ranges

- Broad frequency bandwidth for to allow for flexibility in matching to environmental vibration frequencies which do not generally display narrow or fixed resonance frequencies

- Relatively small form factor when compared to the form factor of other components being used in the transmission line monitoring technology (modems, permanent power sources, processors etc) 
A typical magnetic coupling design proposed as a result of the work in this project is shown in Figure 1-2. A permanent neodynium magnet is attached to the free moving tip of a piezoelectric cantilever beam that is mounted onto a shaker table. A second neodymium magnet is fixed above the cantilever so that the two magnets are attracted by a non-linear force. The cantilever is manufactured using commercially available unimorph piezoelectric discs composed of a $0.9 \mathrm{~nm}$ thick PZT layer deposited on a $1 \mathrm{~mm}$ thick brass shim (APC International, MFT-50T-1.9A1). The disc is cut into a $13 \mathrm{~mm}$ wide by $50 \mathrm{~mm}$ long strip, and clamped at one end to produce a 44 $\mathrm{mm}$ long cantilever. The PZT layer extends $25 \mathrm{~mm}$ along the length of the cantilever, and the remainder is brass only. The proof mass (including the magnet and an additional fixture that holds the magnet) weighs $2.4 \mathrm{gm}$, while the cantilever itself weighs $0.8 \mathrm{gm}$. The electrical leads are carefully soldered with thin lead wires (134 AWP, Vishay) to the top side of the PZT and the bottom side of the shim. Vibration is generated by a shaker table (Labwork ET-126) powered by an amplified sinusoidal wave using a Yokogawa EG300 function generator and a Labwork Pa-13 amplifier. A custom Labview data acquisition program measures output voltage from the cantilever beam.

The movement of the cantilever is monitored by measuring the rectified voltage output across a 1 $\mathrm{M} \Omega$ resistor in parallel with a $2.2 \mathrm{pF}$ capacitor to mimic a device load. Figure 1-3 shows the voltage output integrated over the $15-35 \mathrm{~Hz}$ frequency range. The integrated voltage output increases substantially with decreasing magnet separation, reflecting the broadening of the resonant peak.

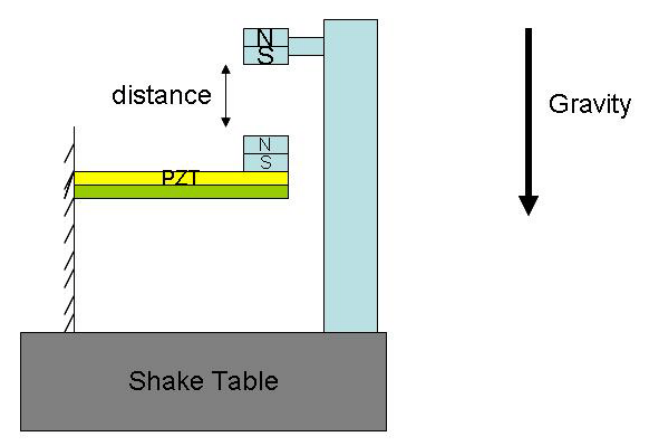

Figure 1-2: Sample schema to achieve voltage amplification and optimal frequency response using a magnetically coupled cantilever scheme for energy harvesting. 


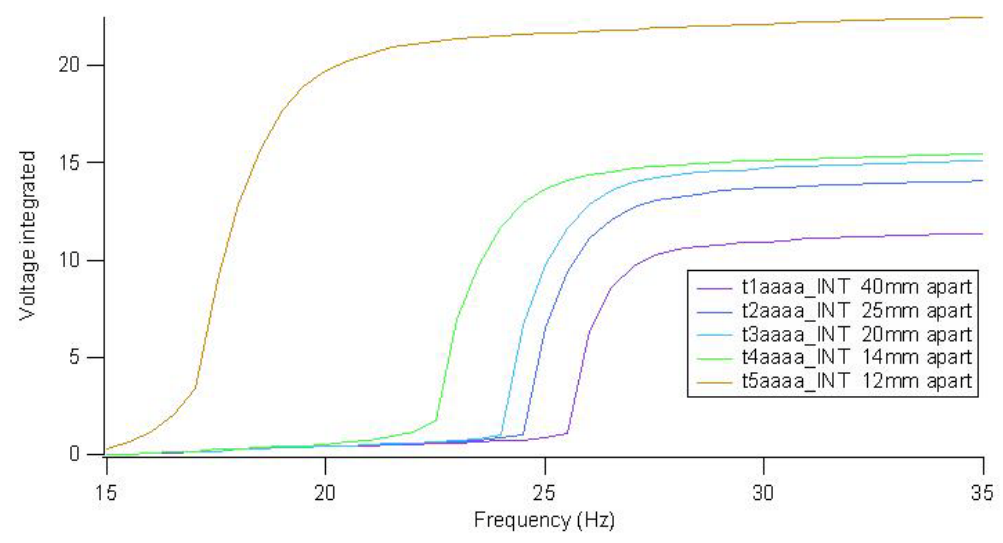

Figure 1-3: Sample voltage amplification as a function of typical environmental outdoor frequencies for an optimal magnetically coupled cantilever scheme such as that shown in Figure 1.2 .

While this scheme can be used to tune the resonant frequency, a magnet placed above the cantilever in this way introduces an asymmetric force, which grows in magnitude as the cantilever approaches the magnet. Because the cantilever deflection is largest at resonance, the influence of the magnetic force is particularly large at the resonance frequency, and damping occurs. By modifying the orientation and magnitude of the magnetic force, we were able to solve this problem. As shown in Figure 1-4, the opposing magnet was moved to the free end of the cantilever to supply a symmetrical, repulsive force about the balance of the cantilever during vibration. The magnetic force drops off with distance away from the equilibrium point, and so has only a minor influence at resonance where the cantilever deflection is relatively large. Outside the resonant frequency, the magnetic force causes the cantilever to undergo chaotic motion between the local minima on either side of the equilibrium point. The response is thus broadened by the appearance of non-periodic oscillations outside of the resonance condition, while the output at the resonant frequency remains unchanged. This provides an effective method to harvest off-resonance vibrations, and increase the output of the piezoelectric cantilever for random or broad-band vibration sources. 


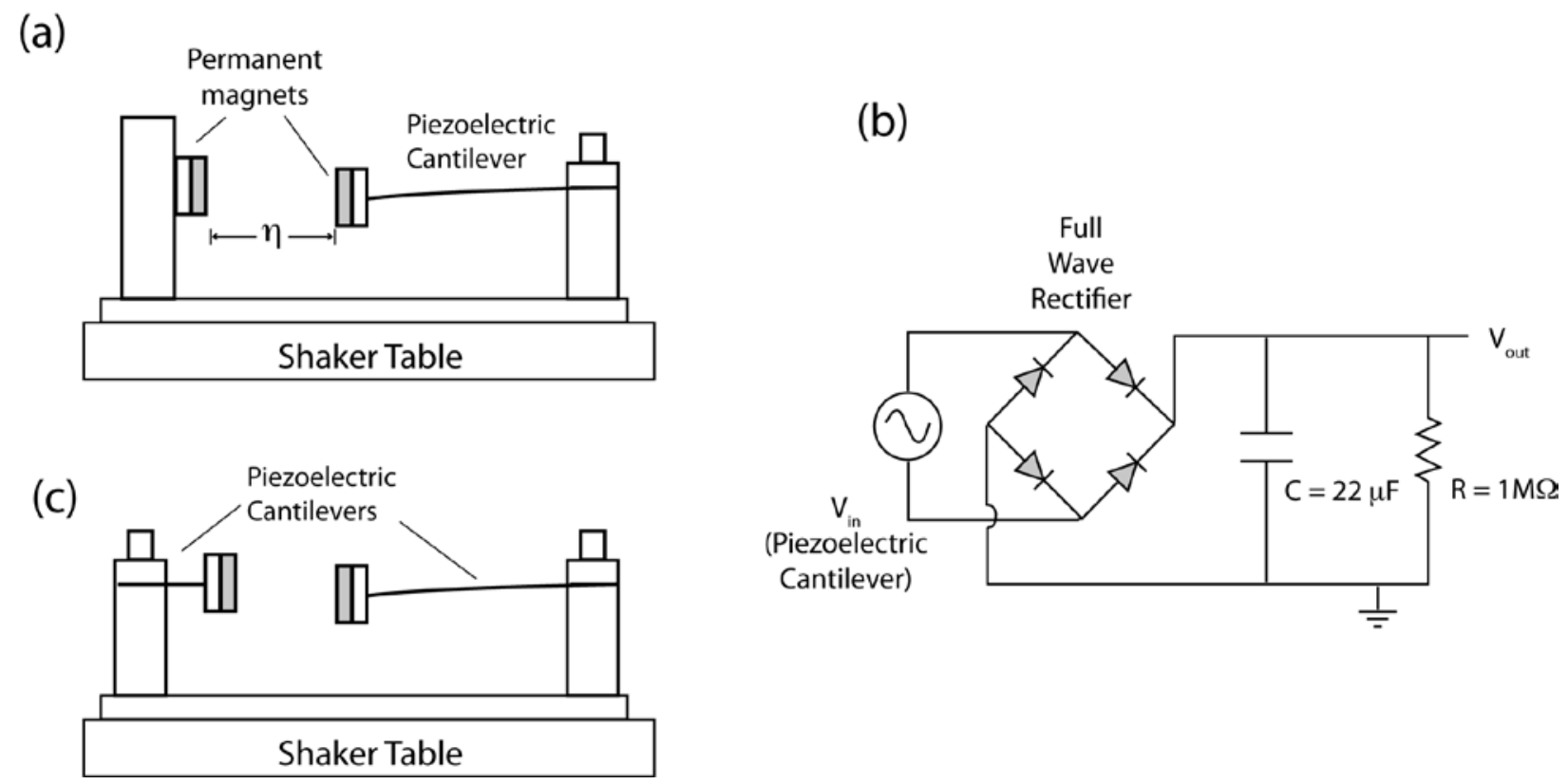

Figure 1-4: (a) Set-up with fixed opposing magnet (b) Measurement circuit (c) Set-up with opposing magnet attached to a second cantilever.

The horizontal separation between the magnets (designated by $\eta$ in Figure 1-4) is adjusted to be approximately $\eta=5 \mathrm{~mm}$. This separation was found to provide the best compensation for the spring force, and made the effective restoring force as small as possible near the equilibrium point. In all measurements, the shaker table acceleration is set to approximately $7 \mathrm{~m} / \mathrm{s}^{2}$, and the frequency swept from 0 to $50 \mathrm{~Hz}$ in $0.5 \mathrm{~Hz}$ steps.

Figure 1-5 (a) shows the output of the cantilever as a function of shaker table vibration frequency for the case where the opposing magnet is fixed to the shaker table. The results from two measurement runs in the coupled state are shown, together with the output of the cantilever measured in the uncoupled state. (This is obtained by removing the opposing magnet.) At the resonance frequency, the output of the cantilever exceeds $16 \mathrm{~V}$, and the peak height, resonance frequency and line width are all approximately the same for the coupled and un-coupled states. On either side of the main resonance, however, there is additional output observed for the coupled cantilever, which is not observed in the uncoupled state. As can be seen from a comparison of the two coupled runs, the frequency distribution of the peaks is not completely reproducible, although there is reproducibility in the overall pattern of the output. 

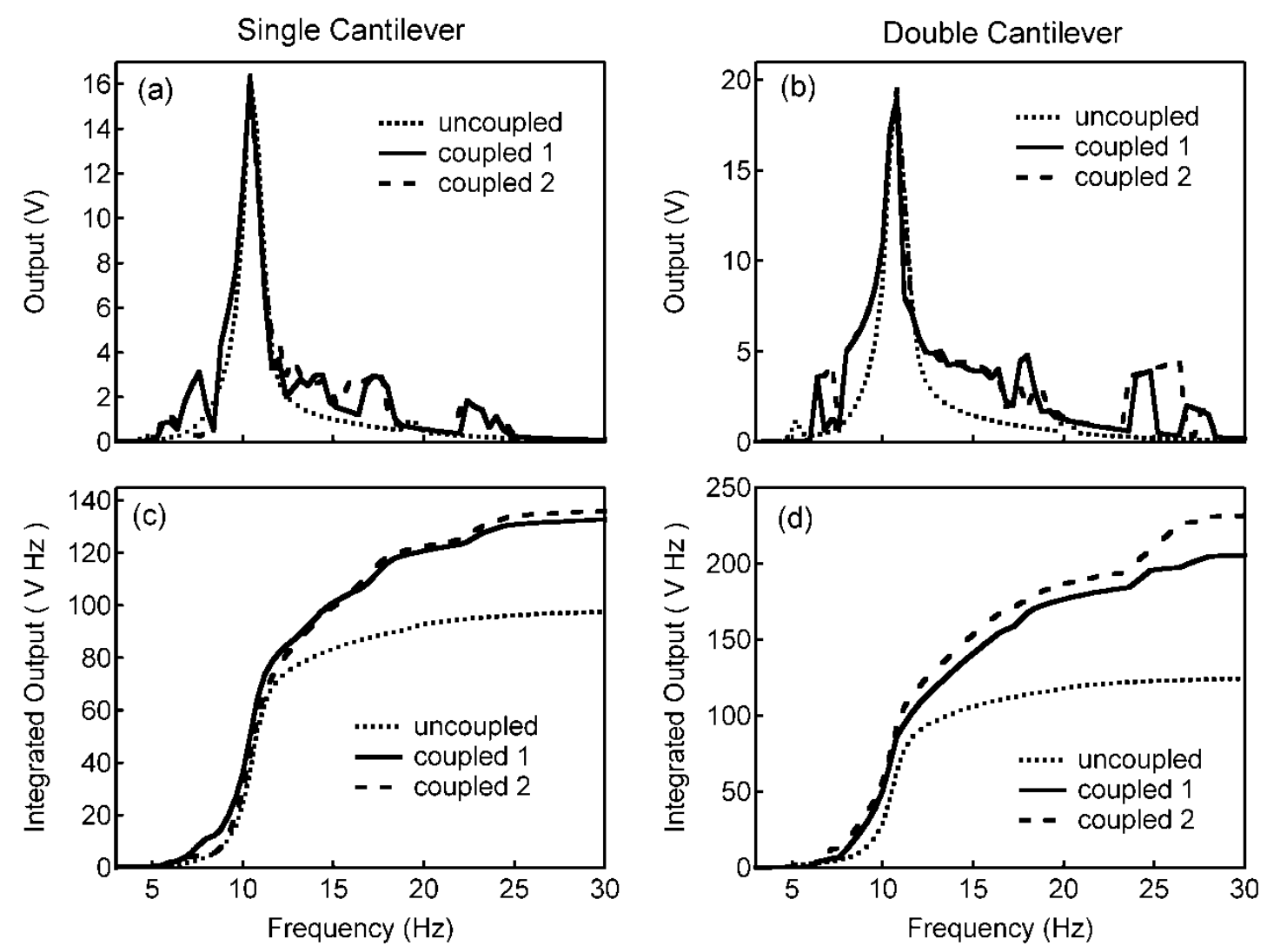

Figure 1-5: Voltage output of the piezoelectric cantilever as a function of shaker table frequency for (a) single cantilever (b) double cantilever. Integrated voltage output as a function of frequency for (c) single cantilever and (d) double cantilever.

Figure 1-6 shows the cantilever output as a function of time measured with the driving frequency at $14 \mathrm{~Hz}$, in the off resonance condition. In this case, the output is measured directly across the cantilever, without rectification or additional load. The uncoupled cantilever motion follows the vibrations of the shaker table, producing periodic oscillations at $14 \mathrm{~Hz}$. For the coupled cantilever, the motion is aperiodic, similar to the chaotic motion observed in a magnetic pendulum, with no constant frequency. The magnitudes of the oscillations, however, are appreciably larger for the coupled cantilever than for the uncoupled cantilever.

Also measured was a double cantilever system, (as shown in Figure 1.5(b)), in which the second magnet is connected to an opposing cantilever (having resonant frequency of around $60 \mathrm{~Hz}$ ) rather than to a fixed point. As shown in Figure 1-6 (b), the results are similar to the single cantilever system, except that the double cantilever system shows a larger overall increase in offresonance output. The overall improvement in the harvesting efficiency can be illustrated by plotting the integrated voltage output of the cantilever beam as a function of frequency. For both 
the single (Figure 1-5 (c)) and double (Figure 1-5 (d)) cantilever systems, the integrated voltage output over the $0-50 \mathrm{~Hz}$ bandwidth shows a substantial increase in the coupled versus the uncoupled case. The total improvement is $31 \%-87 \%$, with some variation between measurement runs.

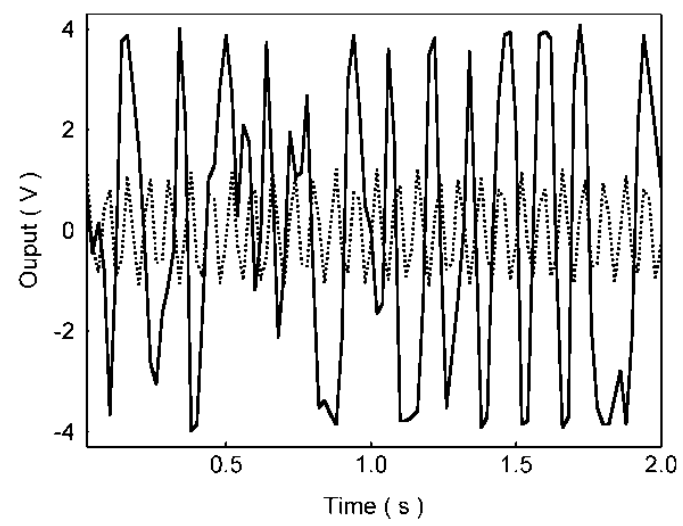

Figure 1-6: Non-rectified voltage output at $14 \mathrm{~Hz}$ vibration for coupled (solid line) and uncoupled (dashed line) cantilever.

We next demonstrated how this capability can be used to improve power output from a broadband vibration source, having a $1 / \mathrm{f}$ frequency dependence (pink noise). A $1 / \mathrm{f}$ vibration spectrum describes a vibration source in which the power spectral density of the vibration is inversely proportional to frequency. Since many naturally occurring vibration sources display a $1 / \mathrm{f}$ dependence, this provides evidence that the magnetic coupling could be used for more efficient energy scavenging in practical settings. The pink noise was generated numerically, with amplitude and crest factor set to $-4 \mathrm{~dB}$ and 1.41 , respectively. The average shaker table acceleration is $7.5 \mathrm{~m} / \mathrm{s}^{2}$, independent of the magnetic coupling. 


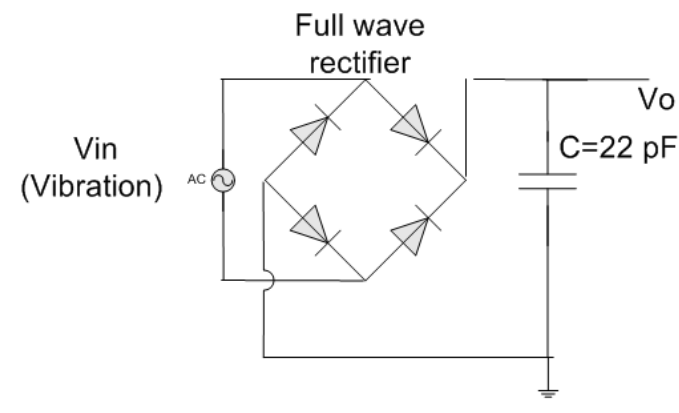

(a)

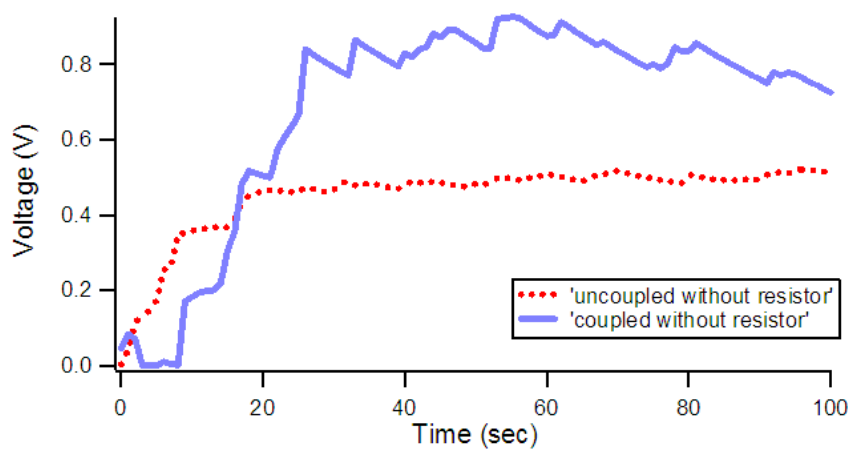

(b)

Figure 4 Lin et al.

Figure 1-7: (a) The schematic of the storage circuit, and (b) DC voltage output measured on the storage capacitor indicating more charge is stored with the magnetic coupling setup.

Figure 1-7 shows the rectified voltage output of the piezoelectric cantilever measured directly across a $22 \mu \mathrm{F}$ capacitor under the influence of the pink noise vibrations. The voltage across the capacitor increases with time, until a maximum charging voltage is achieved. The maximum voltage measured across the capacitor is approximately $50 \%$ higher in the coupled case than in the uncoupled case ${ }^{8,9}$. Note that there is a time delay for the coupled cantilever to achieve a higher voltage than the uncoupled cantilever. This is due to the time passing before the first large amplitude deflection occurs. The random nature of the motion means that this time will vary from run to run, however, on average the coupled cantilever output will be consistently higher than the uncoupled output. Note that in addition to producing more power, the higher voltage output enables circuit operation without a step-up transformer, eliminating the power loss in the transformer.

Further work was directed towards improving the output of the energy scavenging system at low vibrational accelerations, (closer to those experienced in practical settings). Simulated results suggest that low acceleration operation requires that the magnetic force change polarity quickly as the cantilever moves through its equilibrium point. This can be accomplished by reducing the diameter of the fixed magnets attached to the cantilevers. Figure 1-8 shows the integrated output of the piezoelectric cantilever as a function of vibrational acceleration for $5 \mathrm{~mm}, 2 \mathrm{~mm}$, and $1 \mathrm{~mm}$ diameter magnets. In each case, the output with magnetic coupling (blue line) is compared to the output with no coupling (red line). As can be seen, for high accelerations, the output with coupling is always higher than the output without coupling. However, there is a certain threshold acceleration below which the magnetically coupled output drops below the uncoupled output. Ideally, this threshold voltage should be as low as possible. The experiments show that by reducing the diameter of the magnets it is possible to reduce the threshold below $1 \mathrm{~m} / \mathrm{s}^{2}$, a value approaching the vibrations observed in standard environmental situations. To decrease the 
thickness of the magnets further, the magnetic layer must be deposited directly onto the cantilever. Work is currently underway to develop a procedure for magnetic material deposition by either sputtering or e-beam evaporation.
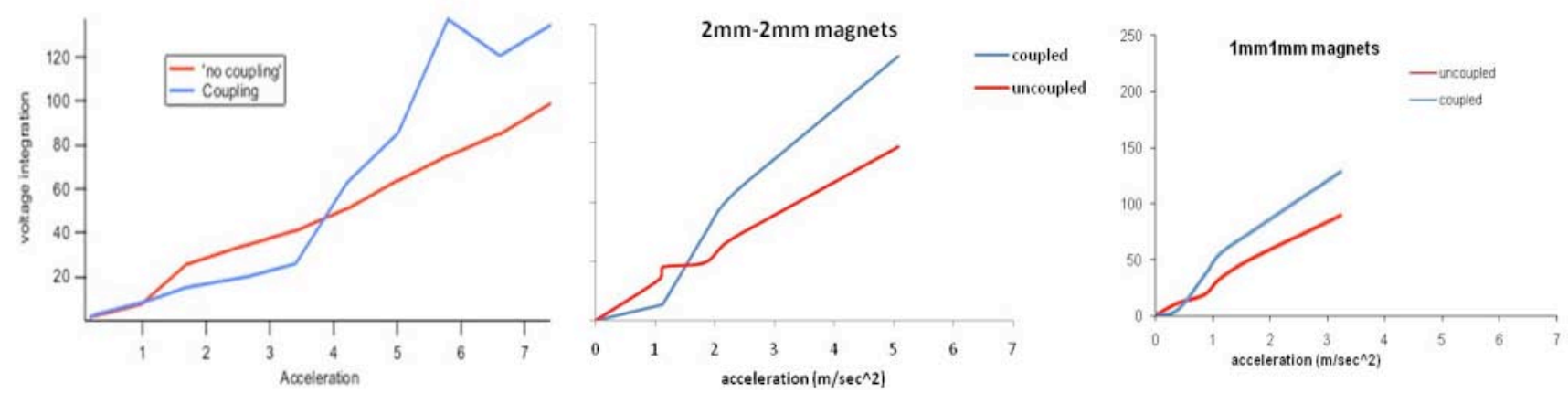

Figure 1-8: Integrated voltage output versus vibration acceleration magnitude for three different diameter magnets. In each case, the coupled (blue line) is compared to the uncoupled (red line).

\section{Theoretical Modeling of Magnetically Coupled Cantilever Designs}

If a piezoelectric material undergoes a periodic stress due to external vibrations (Figure 1-9), an alternating open circuit voltage can be measured across the material given by

$$
\text { Voc }=-\delta \mathrm{t} \sigma / \varepsilon
$$

where $\boldsymbol{\delta}$ is the strain coefficient, $\mathbf{t}$ is thickness, $\boldsymbol{\sigma}$ is stress, and $\boldsymbol{\varepsilon}$ is dielectric constant. The output voltage, resultant cantilever strain can be modeled as a function of applied acceleration on the cantilever $\ddot{\mathbf{y}}$ by

$$
\begin{aligned}
& \delta \mathbf{t t}=-\mathbf{A} \delta-\mathbf{B} \delta \mathbf{t}+\mathbf{C V}+\mathbf{D} \ddot{\mathbf{y}} \\
& \mathbf{V t}=-\mathbf{E} \delta \mathrm{t}
\end{aligned}
$$

where $\delta \mathrm{tt}$ is strain acceleration, $\mathrm{Vt}$ is change in voltage, A,B,C,D and $\mathrm{E}$ are constants that depend on the mass $\mathrm{M}$, cantilever dimensions, and piezoelectric properties. 


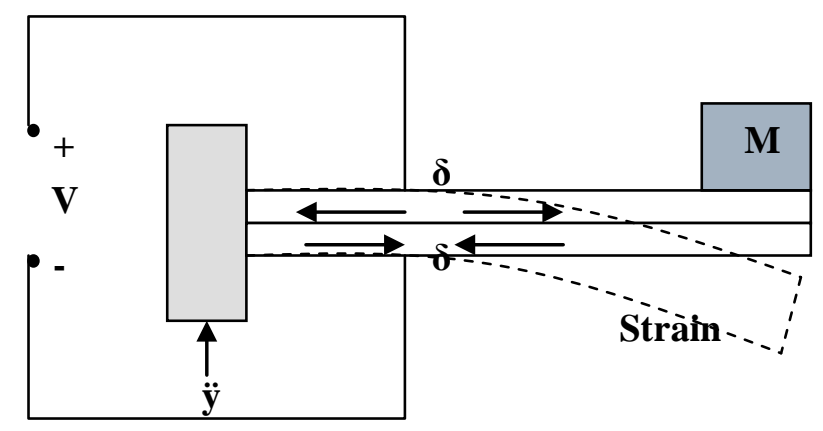

Figure 1-9: Model of the strained piezoelectric cantilever

To calculate the amplitude of the cantilever deflection in the presence of the magnetic coupling force, we use a modified version of a standard spring-mass model. The model parameters are shown schematically in Figure 1-10(a). The cantilever is represented by a one-dimensional spring-mass system, where $m$ is the proof mass, and $k$ is the spring constant. The cantilever deflection, $z(t)$, is driven by a vibrating source, which oscillates sinusoidally with an acceleration $A$ and angular frequency $\omega$. Electrical and parasitic damping are taken together as a single damping coefficient, $d$.
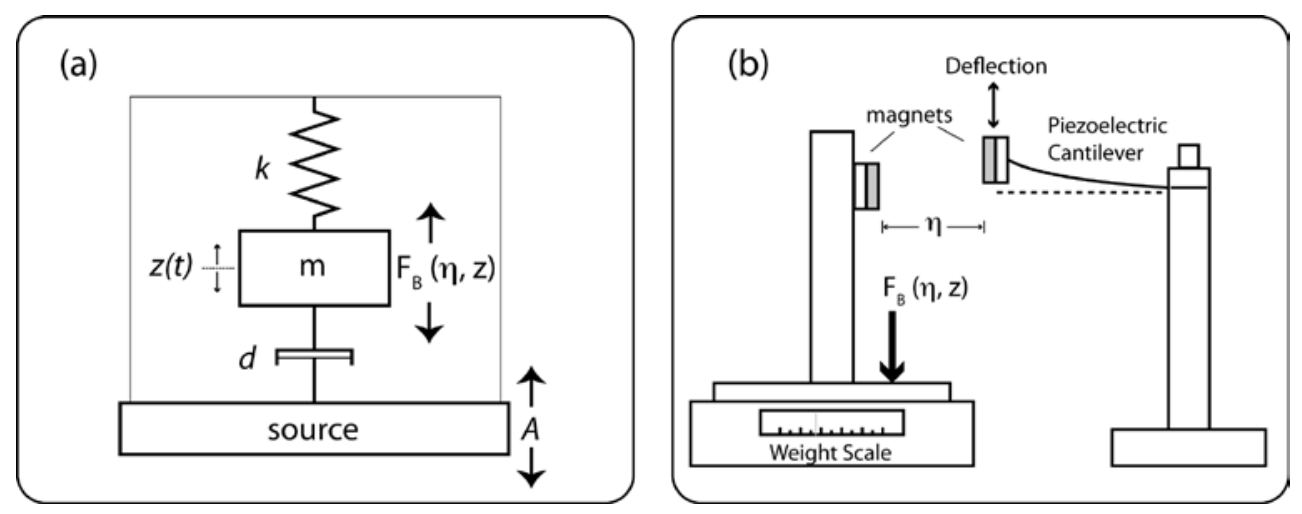

Figure 1-10: (a) Modified one-dimensional spring force model. A is the source acceleration, $\mathrm{k}$ is the spring constant, $m$ is the proof mass, $z(t)$ is the cantilever deflection and $F_{B}(\eta, z)$ is the magnetic force. (b) Apparatus used to measure the magnetic force $F_{B}(\eta, z)$.

To minimize the complexity of the problem, the magnetic coupling force $F_{B}$, is considered to be one-dimensional, acting only in the z-direction. The deflection $z(t)$ can then be determined by 
solving the differential equation for a one-dimensional forced harmonic oscillator, combined with an unknown non-linear force:

$$
\ddot{z}(t)+\frac{d}{m} \dot{z}(t)+\frac{k}{m} z(t)+F_{B}(z, \eta)=A \cos (\omega t)
$$

In general, the magnetic coupling force $F_{B}(z, \eta)$ is a complicated non-linear function of the deflection $z$ and the magnet / magnet separation distance, $\eta$. However, for a given value of $\eta$, the force component in the z-direction can be determined experimentally by measuring the weight change of the cantilever under manual deflection. Figure 1-10(b) shows the experimental set-up for the force determination. The opposing magnet is mounted onto a weighing scale, and the separation between the magnets $\eta$ is measured at the balance point $(\mathrm{z}=0)$ when the magnets are in line with each other. The position of the magnetized cantilever is then manipulated by pushing up and down at the end of a cantilever beam, simulating flexure movement. The deflection $z$ is measured using a micrometer, while the reading on the scale provides the force between the two magnets. The cantilever's restoring force is determined independently by setting the cantilever on the weighing scale, deflecting the cantilever, and recording the weight / deflection relationship.
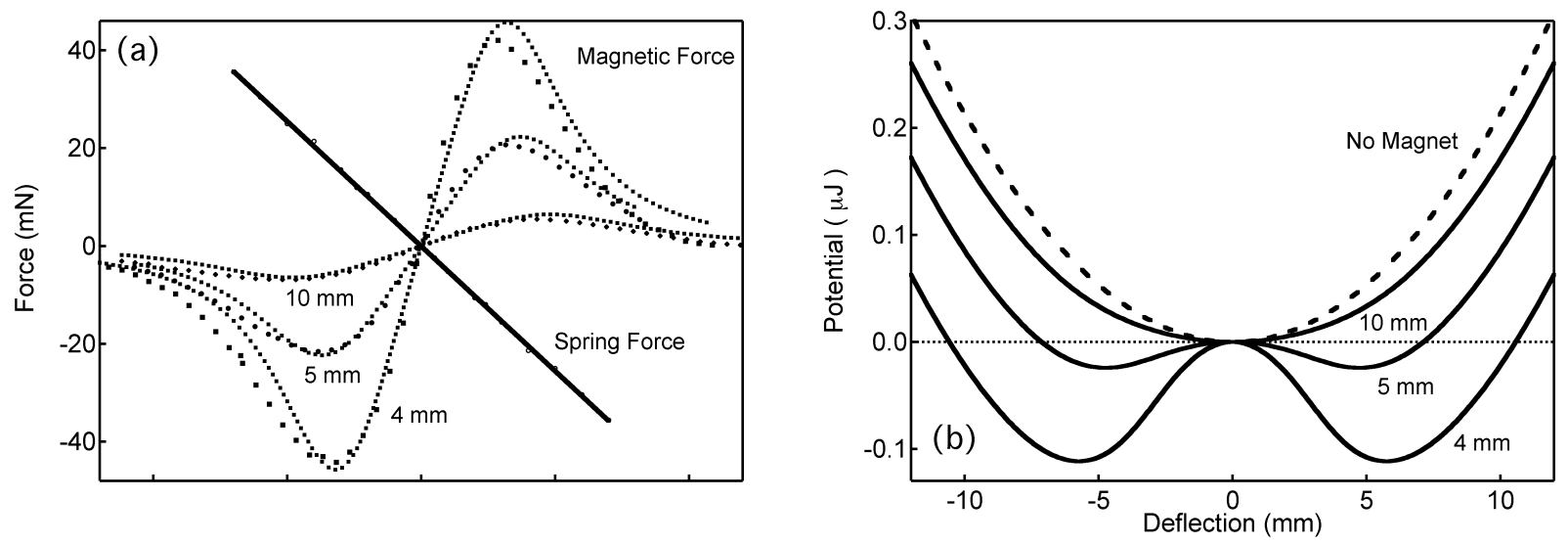

Figure 1-11: (a) The magnetic force $F_{B}(\eta, z)$ for three separation distances $(\eta=4 \mathrm{~mm}, 5 \mathrm{~mm}$ and $10 \mathrm{~mm}$ ). The lines show fits to Equation 1.4. Also shown (as a straight line) is the spring force of the cantilever. (b) The cantilever spring potential (dashed line) and the potential due to the combination of the restoring force and the magnetic force for the 3 separation distances.

The magnetic forces $F_{B}(z, \eta)$ determined for three different magnet separation distances $\eta$ are plotted in Figure 1-11 as a function of the deflection distance $z$. For all three values of $\eta$, two maxima are observed in the magnetic force magnitude, at approximately $\mathrm{z}=+/-5 \mathrm{~mm}$. This deflection distance is equal to the magnet diameter, and corresponds to the point where the two magnets no longer overlap each other. The magnitude of the maxima increase rapidly with 
decreasing $\eta$. To aid in calculation, the experimentally determined magnetic force values are fit to an empirically determined analytical expression for FB (zๆ):

$$
F_{B}(z, \eta)=\frac{a z}{\left(b+c z^{4}\right)},
$$

where a, b, and c are fitting parameters. As shown in Figure 1-11, this ad hoc expression provides a reasonably accurate fit to the magnetic force data. The influence of the magnetic force can be better appreciated by considering the potential energy of the cantilever as a function of deflection distance, as shown in Figure 1-11(b). The potential energy is determined by integrating over the total force (magnetic force plus restoring force), where the analytical expression for the magnetic force (given by Equation 1.5) is used. As seen in Figure 1-11(b), the magnetic force modifies the standard harmonic oscillator potential to include two potential minima, one on either side of the zero deflection point, where it is equally likely for the cantilever to lie in either of these minimum points. For large enough magnet separations, motion between the two minima is possible under relatively low amplitude accelerations and at nonresonance driving frequencies.

Using the analytic expression for the magnetic force, it is possible to simulate the output of the magnetically coupled cantilever. As described in Roundy, Wright and Rabaey, the electrical power produced by a vibrating spring-mass system is given by:

$$
P=\frac{1}{2} b_{e} \dot{\bar{z}}^{2}
$$

where $z$ is the spring deflection, and $b_{e}$ is the electrically induced damping coefficient. The instantaneous power at time $t$ is thus proportional to $\dot{z}^{2}$, which can be determined numerically from Equation 1.

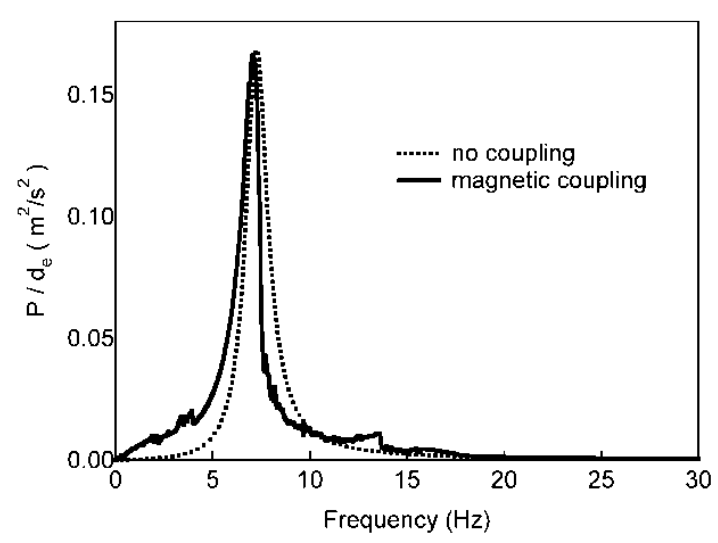

Figure 1-12: Simulated output of the piezoelectric cantilever for the case of no magnetic coupling (dashed line) and magnetic coupling (solid line). 
In our calculation, we evaluate $\dot{z}(t)$ at ms time intervals for $2 \mathrm{~s}$ and take the average of these values to calculate the time averaged power, normalized by the damping coefficient $\left(P / b_{e}\right)$. Figure 1-12 shows the results of this calculation as a function of driving frequency for both the coupled and uncoupled cantilevers. In the absence of magnetic coupling, a peak in the output is observed at the resonance frequency. The addition of magnetic coupling produces additional output above and below the resonant frequency, while little change is observed in the resonance peak. This is in qualitative agreement with the experimental observations. The parameters used in the simulation were identical to those used in the experiment. Further work is needed to determine the exact range of validity of the model.

\section{Magnetic Field Scavenging}

Since the electric transmission line monitor will be located in areas with relatively high magnetic fields, it may be possible for a federal agency to obtain permission from grid owners and operators to use the magnetic field at these sites to aid in powering devices as a standalone method or to complement other environmental energy scavenging schemes. Some basic experiments were conducted to determine whether the magnetic field surrounding overhead transmission lines can be used as a potential source for power scavenging for the transmission line monitor. Magnetic solenoids which produce an output voltage of $10 \mathrm{mV} / \mathrm{milliGauss}$ were tested in conjunction with a series of circuit designs to harvest this voltage. Typical fields close to high voltage transmission lines vary from 20-60 milliGauss. This suggests that voltages ranging from 0.1 to $0.6 \mathrm{~V}$ are possible under typical transmission line conditions.

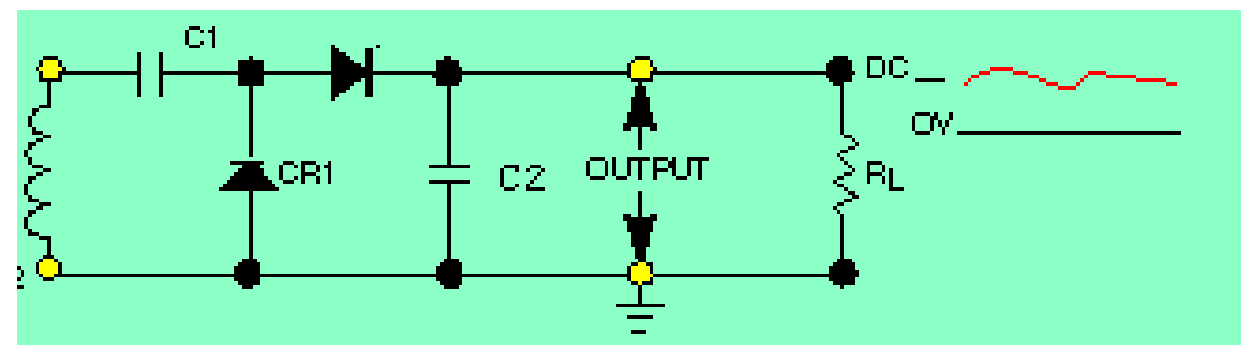

Figure 1-13: Voltage doubling circuit to maximize voltage output from induced voltage on the pick-up solenoid in the presence of a typical magnetic field measured under a high voltage transmission line.

A voltage doubling circuit such as the one shown in Figure 1-13 has been shown to produce a maximum voltage of $0.6 \mathrm{~V}$ using the solenoid coil in a field of 20 milliGauss.

Based on the work performed here, novel energy scavenging methods such as vibration scavenging and magnetic coupling present a real possibility for powering wireless units for transmission line monitoring. The work performed here concentrated on optimization of single cantilever designs for vibration scavenging. A next stage of this work would involve the design 
and development of multiple unit arrays in order to multiply the output of one device until desired power levels for required data transmission is achieved.

\section{Research and Design of Methods for Utilizing Directional Sensors}

Genscape, a Kentucky based energy information provider has developed patented systems and methodologies to determine power flow in high voltage electric transmission power lines since 2000. The company has a network of approximately 1400 wireless transmission line monitors deployed in the United States. Genscape network installations are primarily in rural locations where power lines are generally isolated from other power lines. However, power line monitoring applications for grid situational awareness is an application which requires a power line measurement method to be equally effective in high power line density situations as well as low density power line situations. In order to achieve this goal, methods were investigated to uniquely determine electromagnetic fields in cases where power lines exist in close proximity to a number of other power lines.

\subsection{Multiple Line Sensing - Tri-axial (or directional) magnetic field sensors}

\subsubsection{Power Line Modeling}

A 3-axis sensor was investigated for distinguishing electromagnetic fields produced from power lines in close proximity. Mathematical modeling of various line configurations (Figure 2-1) and bench top simulations of power line configurations were used to optimize sensor placement and determine signal processing techniques required to identify and filter out magnetic field contributions from specific lines of interest.

The superposition of electromagnetic fields from neighboring power lines and variations in the resultant angle of the superimposed magnetic field vector was modeled. In the example shown in Figure 2-1 below, two lines are separated by a distance of 60 feet. The magnetic sensor is positioned 45 feet from left line and 15 feet from the right line. The magnetic vector angles were calculated for three cases

1. Power flow on right line only

2. Power flow on left line only 
3. Equal power flow on both lines

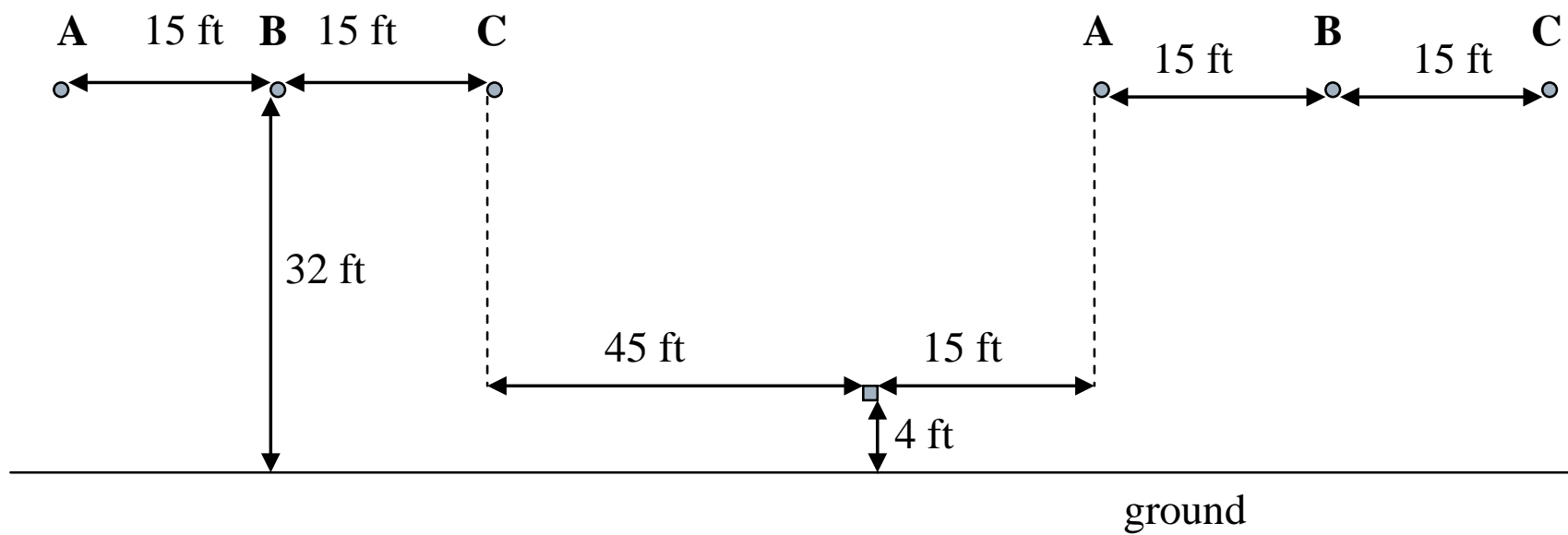

a.

b.

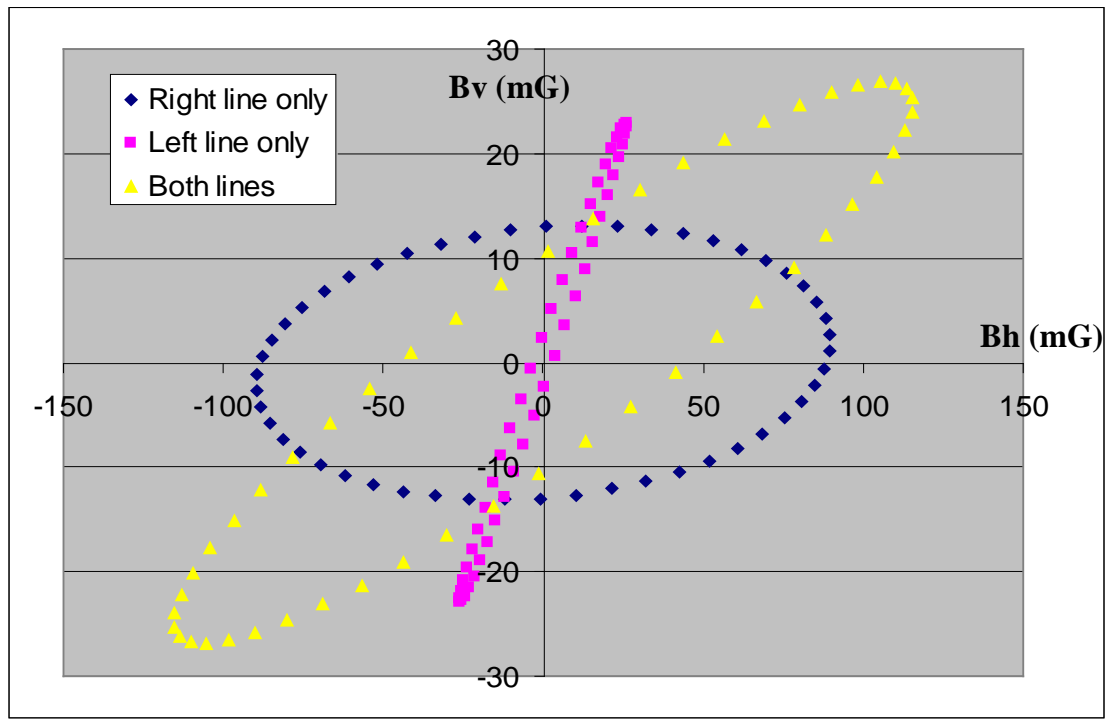

Figure 2-1: a. Line configuration example for two transmission lines separated by 60 feet. Sensor is placed 45 feet from left line and 15 feet from the right line. b. The resultant magnetic field vector for three different power flow distributions (power flow on right line only, power flow on left line only and equal power flow on both lines).

The model suggests that there is a variation in the angle of vector rotation for the three cases. A laboratory transmission line simulation was developed as shown in Figure 2-2(a) to test the modeling results presented above. An inverter is used to deliver 3 phase power along 3 parallel conductors. An LCR filter is used to produce a smooth $60 \mathrm{~Hz}$ sine wave output. The goal of this experiment was to confirm the validity of the theoretical calculations. Initial results using the 
model transmission line shows that the axis of rotation of the magnetic field vector varies with distance from the line as shown in Figure 2-3. Various line positions and power distribution configurations were tested as shown in Figure 2-2(b). The agreement between the simulation and experiment suggests that acquisition of data acquired on the full magnetic field vector using the 3 -axis sensor can distinguish varying power levels on transmission lines in close proximity to each other.

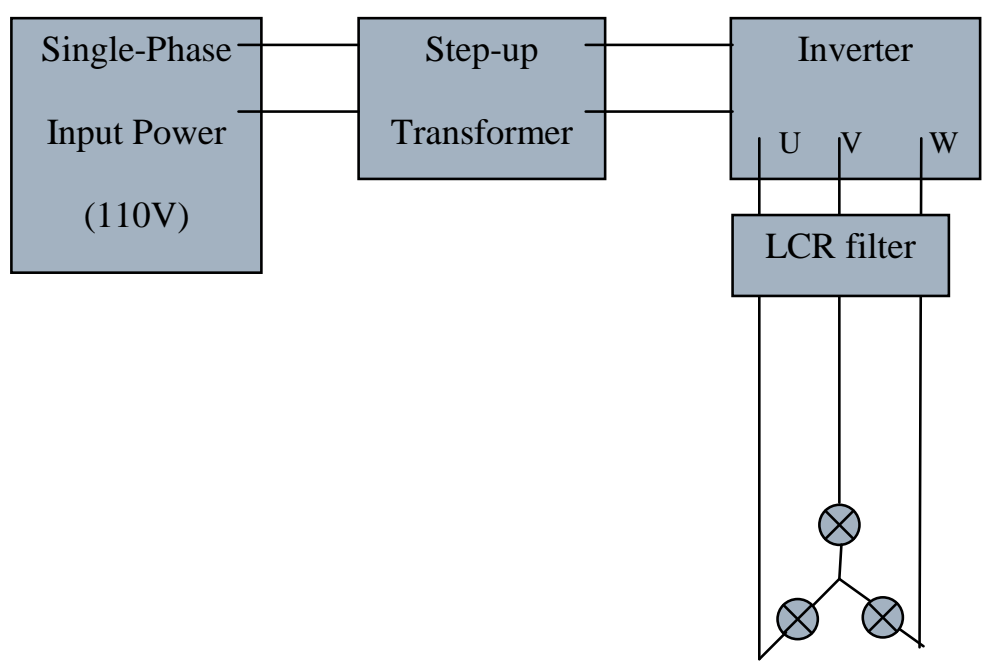

a.

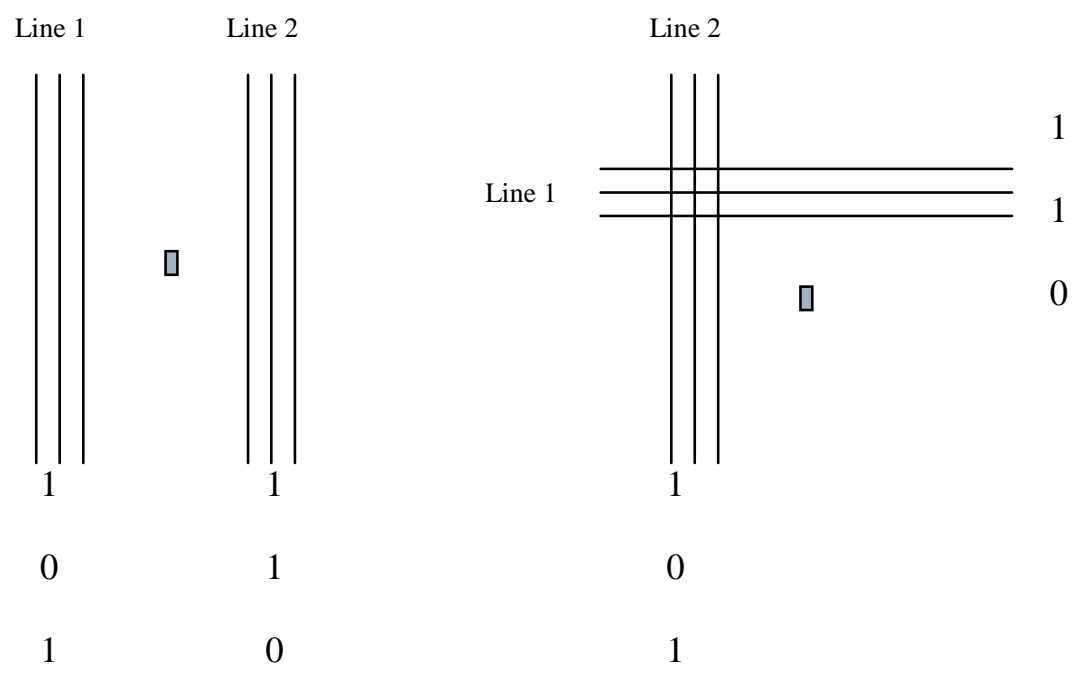

b. 
Figure 2-2: a. Circuit design for simulated power line electromagnetic field testing in the laboratory. b. Line configurations and power distributions tested using the laboratory simulation.

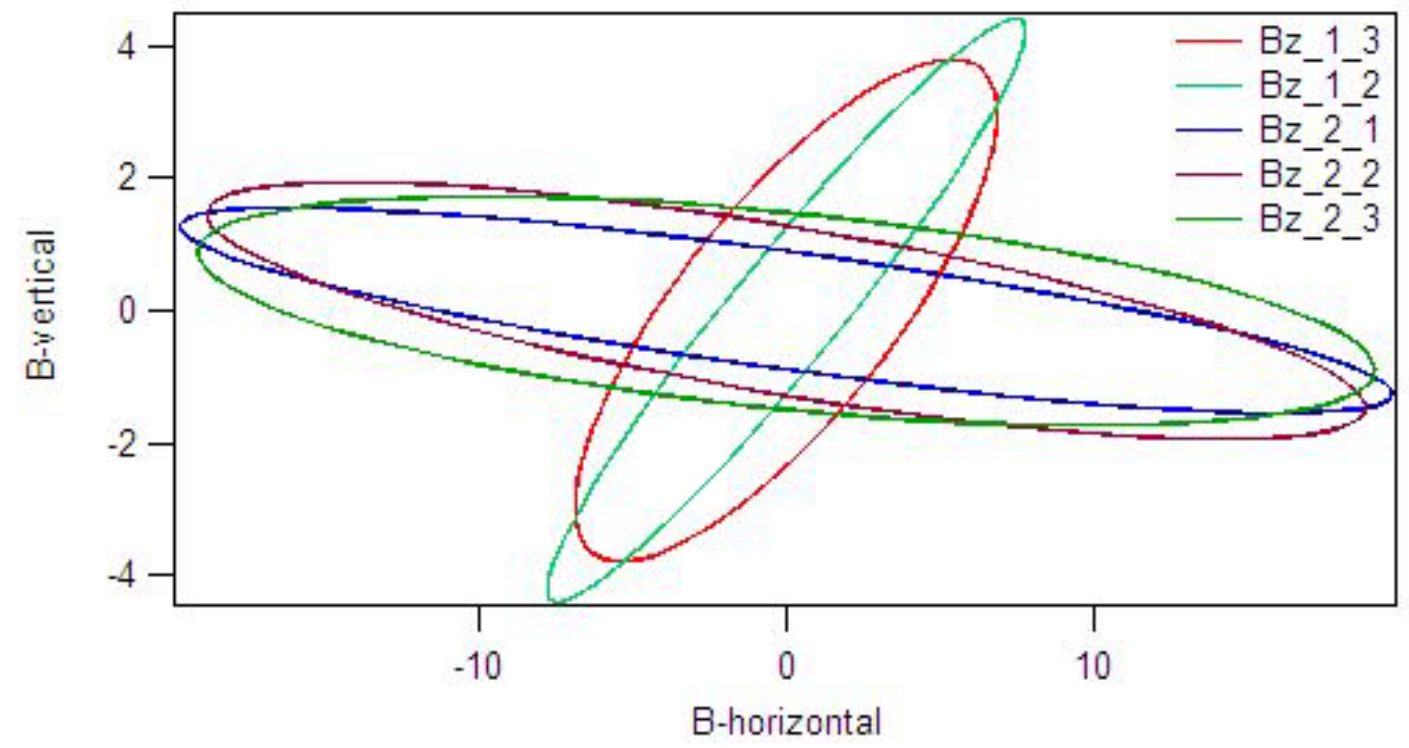

Figure 2-3: Comparison between the angle of vector rotation under the model line (Bz_2 mG) and $4 \mathrm{~cm}$ from the model line $\left(\mathrm{Bz} \_1 \mathrm{mG}\right)$

\subsubsection{Directional Sensing Techniques}

Figure 2.4 shows test transmission line configurations where the magnetic field from power line Line 2 interferes with the measurement of a line of interest, power line 1. A number of possible monitor locations for each scenario were modeled in order to develop techniques to measure power flow and direction in these cases. 


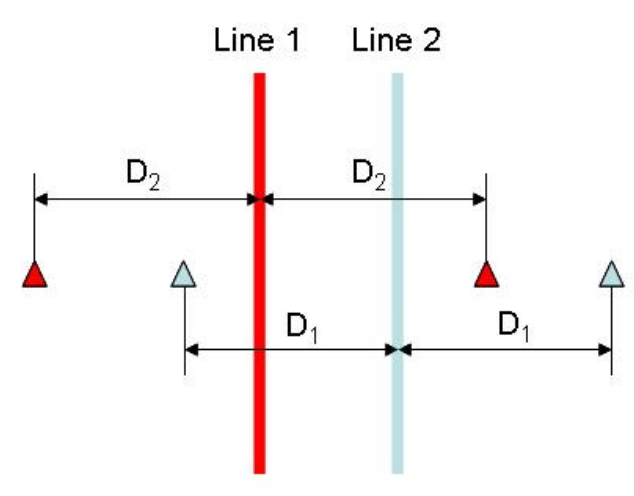

$\triangle$ : Monitor

a.

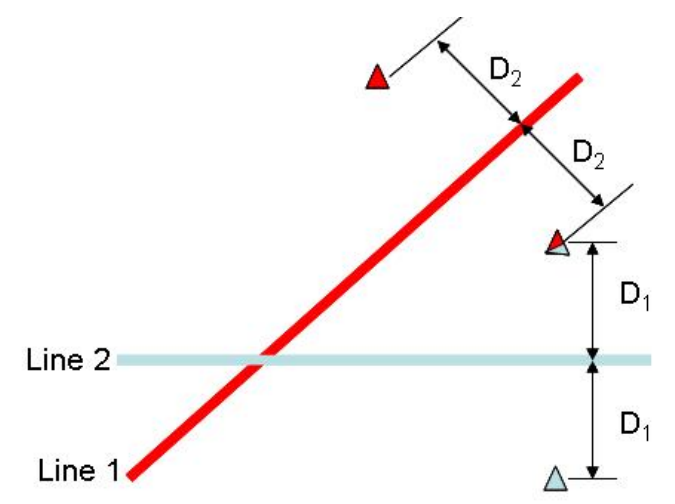

$\triangle$ : Monitor

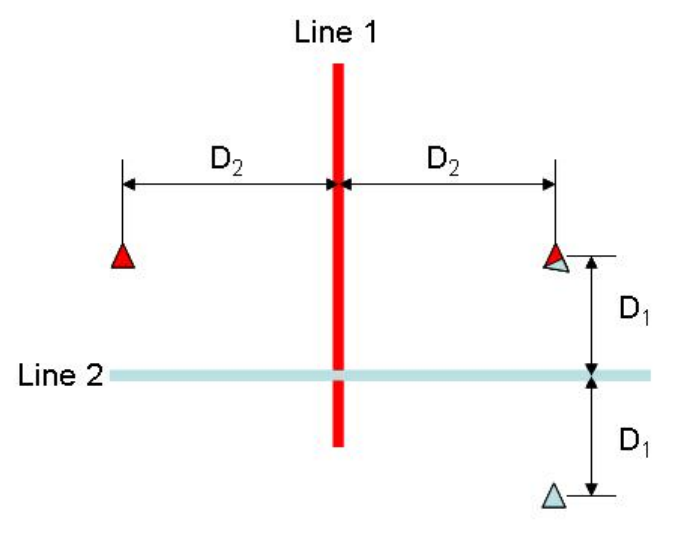

$\triangle:$ Monitor b. 
Figure 2-4 a. Parallel, b. perpendicular and c. crossed transmission lines. The indicated monitor locations were tested as solutions to provide a method of measuring power flow on each line independently.

A technique investigated for the cases illustrated in Figure 2.4 involves the cancellation of fields by placing monitors symmetrically about the center of each line and using field cancellation to isolate EMF signals from a particular line of interest. In order to explore the cancellation of EMF field values from two monitors which are placed symmetrically about a transmission line, the magnetic field vectors in the case of a horizontal single line configuration are computed as follows;

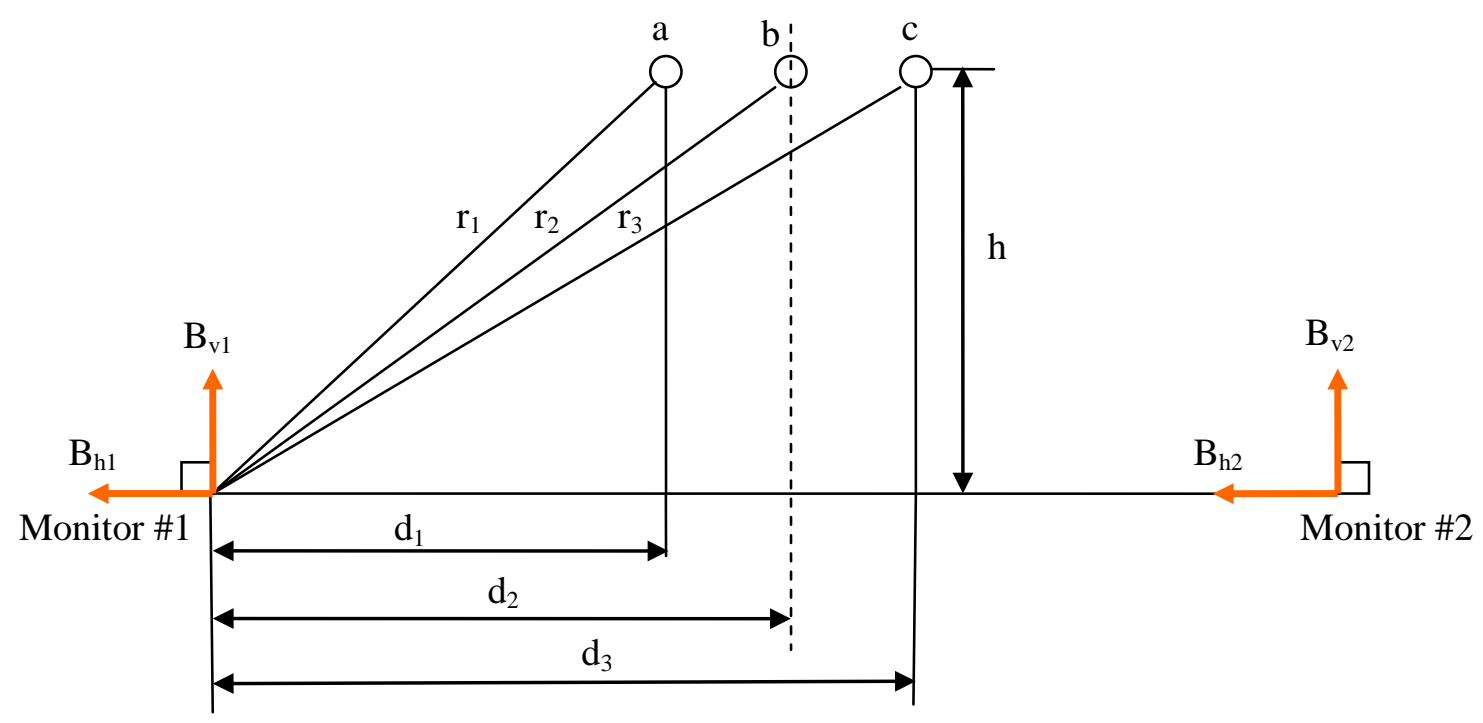

Figure 2-5: Schematic drawing of two monitors symmetrically placed with respect to the centerline of the transmission line (dotted line).

The horizontal and vertical magnetic field components at Monitor 1 are;

$$
\begin{aligned}
& B_{h 1}=B_{h a 1}+B_{h b 1}+B_{h c 1} \\
& B_{h 1}=\frac{h}{r_{1}} \cdot \frac{2 \cdot I_{a}}{r_{1}}+\frac{h}{r_{2}} \cdot \frac{2 \cdot I_{b}}{r_{2}}+\frac{h}{r_{3}} \cdot \frac{2 \cdot I_{c}}{r_{3}}
\end{aligned}
$$




$$
\begin{aligned}
& B_{v 1}=B_{v a 1}+B_{v b 1}+B_{v c 1} \\
& B_{v 1}=\frac{d_{1}}{r_{1}} \cdot \frac{2 \cdot I_{a}}{r_{1}}+\frac{d_{2}}{r_{2}} \cdot \frac{2 \cdot I_{b}}{r_{2}}+\frac{d_{3}}{r_{3}} \cdot \frac{2 \cdot I_{c}}{r_{s}}
\end{aligned}
$$

Similarly the horizontal and vertical magnetic field components at Monitor 2 are;

$$
\begin{aligned}
& B_{h 2}=\frac{h}{r_{3}} \cdot \frac{2 \cdot I_{a}}{r_{3}}+\frac{h}{r_{2}} \cdot \frac{2 \cdot I_{b}}{r_{2}}+\frac{h}{r_{1}} \cdot \frac{2 \cdot I_{c}}{r_{1}} \\
& B_{v 2}=-\left(\frac{d_{3}}{r_{3}} \cdot \frac{2 \cdot I_{a}}{r_{3}}+\frac{d_{2}}{r_{2}} \cdot \frac{2 \cdot I_{b}}{r_{2}}+\frac{d_{1}}{r_{1}} \cdot \frac{2 \cdot I_{c}}{r_{1}}\right)
\end{aligned}
$$

From Equation 2.1and Equation 2.3,

$$
B_{h 1}-B_{h 2}=2 I_{a}\left(\frac{h}{r_{1}^{2}}-\frac{h}{r_{3}^{2}}\right)+2 I_{c}\left(\frac{h}{r_{3}^{2}}-\frac{h}{r_{1}^{2}}\right)=2\left(I_{a}-I_{c}\right)\left(\frac{h}{r_{1}^{2}}-\frac{h}{r_{3}^{2}}\right)
$$

From Equation 2.2 and Equation 2.4

$$
B_{v 1}+B_{v 2}=2 I_{a}\left(\frac{d_{1}}{r_{1}^{2}}-\frac{d_{3}}{r_{3}^{2}}\right)+2 I_{c}\left(\frac{d_{3}}{r_{3}^{2}}-\frac{d_{1}}{r_{1}^{2}}\right)=2\left(I_{a}-I_{c}\right)\left(\frac{d_{1}}{r_{1}^{2}}-\frac{d_{3}}{r_{3}^{2}}\right)
$$

From Equation 2.5 and Equation 2.6, a cancellation of magnetic vector values at both monitors symmetrically placed about the line can be computed by;

$$
\left(B_{h 1}-B_{h 2}\right)\left(\frac{d_{1}}{r_{1}^{2}}-\frac{d_{3}}{r_{3}^{2}}\right)-\left(B_{v 1}-B_{v 2}\right)\left(\frac{h}{r_{1}^{2}}-\frac{h}{r_{3}^{2}}\right)=0
$$

The validity of this cancellation computation was tested in the field on a horizontal single line set.

Figure 2.6 shows sample data obtained from the magnetic field sensors of two monitors placed symmetrically about the line. Figure 2.7 shows the computed cancellation. Here modified $\mathrm{Bv}$ 
represnts $\left(B_{v 1}-B_{v 2}\right)\left(\frac{h}{r_{1}^{2}}-\frac{h}{r_{3}^{2}}\right)$, modified Bh represents $\left(B_{h 1}-B_{h 2}\right)\left(\frac{d_{1}}{r_{1}^{2}}-\frac{d_{3}}{r_{3}^{2}}\right)$ and signal after cancellation is $\left(B_{h 1}-B_{h 2}\right)\left(\frac{d_{1}}{r_{1}^{2}}-\frac{d_{3}}{r_{3}^{2}}\right)-\left(B_{v 1}-B_{v 2}\right)\left(\frac{h}{r_{1}^{2}}-\frac{h}{r_{3}^{2}}\right)$.

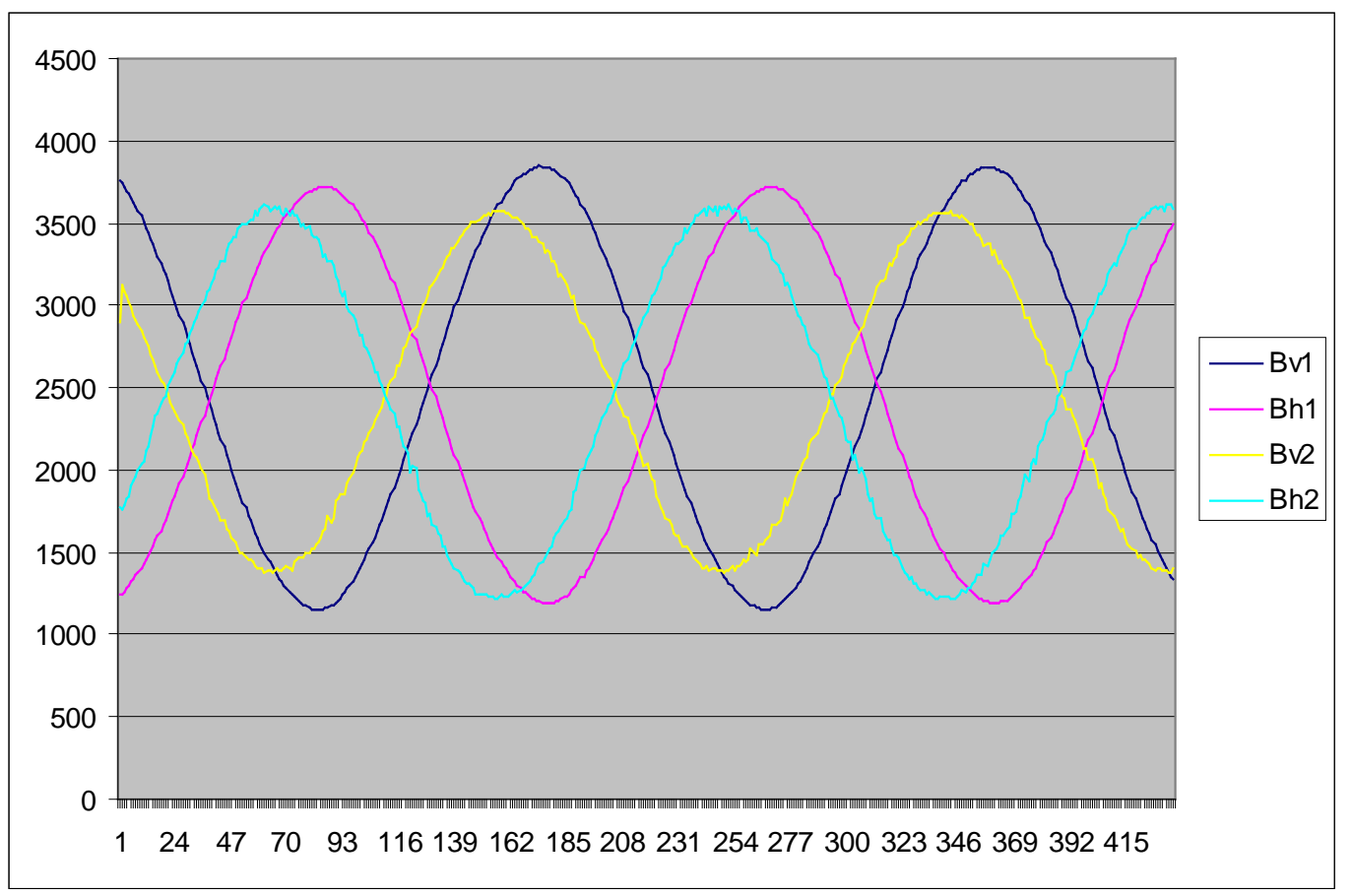

Figure 2-6: Sample magnetic field measurement by two monitors (1, 2). Five $60 \mathrm{~Hz}$ cycles (amplitude in arbitrary analog-to-digital conversion units) are shown. 


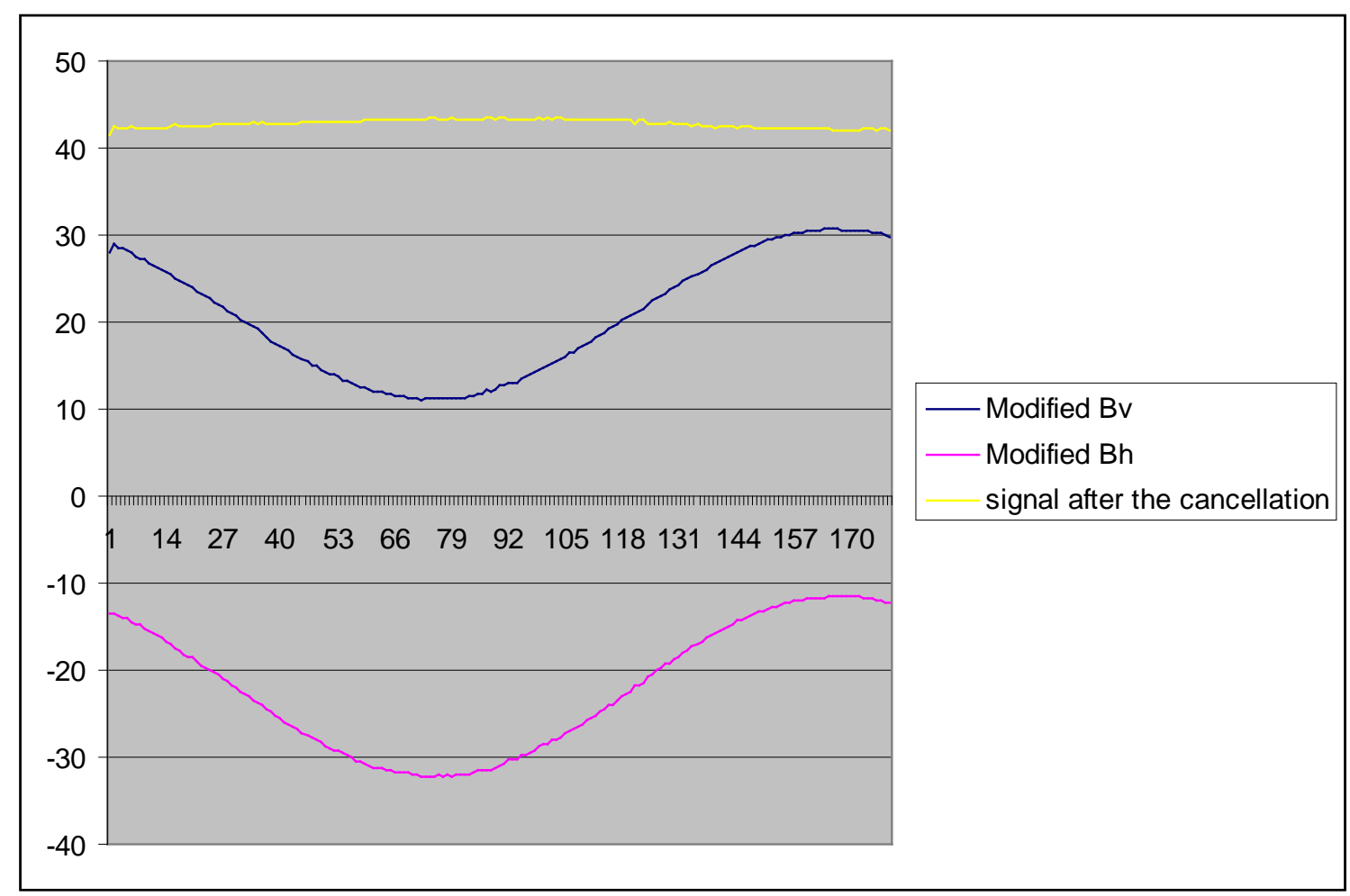

Figure 2-7: Computed values for Equations 2.5, 2.6, 2.7 using actual field measurements for a high voltage overhead transmission line. The computed cancellation is non-zero due to variations in measured values from the theoretically computed values (milliGauss).

Now consider two conductors in parallel as shown in Figure 2.8. To calculate the power flow on transmission line A, two monitors are installed, each symmetric with respect to transmission line B. By separating the induced magnetic field caused by line A and B on two monitors, it can be obtained,

$$
\begin{aligned}
& B_{h 1}=B_{h A 1}+B_{h B 1} \\
& B_{v 1}=B_{v A 1}+B_{v B 1} \\
& B_{h 2}=B_{h A 2}+B_{h B 2} \\
& B_{v 2}=B_{v A 2}+B_{v B 2}
\end{aligned}
$$

From Equation 2.8, we obtain, 


$$
\begin{gathered}
B_{h 1}-B_{h 2}=\left(B_{h A 1}-B_{h A 2}\right)+\left(B_{h B 1}-B_{h B 2}\right) \\
B_{v 1}-B_{v 2}=\left(B_{v A 1}-B_{v A 2}\right)+\left(B_{v B 1}-B_{v B 2}\right) \\
\left(B_{h 1}-B_{h 2}\right)\left(\frac{d_{1}}{r_{1}^{2}}-\frac{d_{3}}{r_{3}^{2}}\right)-\left(B_{v 1}-B_{v 2}\right)\left(\frac{h}{r_{1}^{2}}-\frac{h}{r_{3}^{2}}\right) \\
=\left(\left(B_{h A 1}-B_{h A 2}\right)\left(\frac{d_{1}}{r_{1}^{2}}-\frac{d_{3}}{r_{3}^{2}}\right)-\left(B_{v A 1}-B_{v A 2}\right)\left(\frac{h}{r_{1}^{2}}-\frac{h}{r_{3}^{2}}\right)\right)+\left(\left(B_{h B 1}-B_{h B 2}\right)\left(\frac{d_{1}}{r_{1}^{2}}-\frac{d_{3}}{r_{3}^{2}}\right)-\left(B_{v B 1}-B_{v B 2}\right)\left(\frac{h}{r_{1}^{2}}-\frac{h}{r_{3}^{2}}\right)\right) \\
=\left(\left(B_{h A 1}-B_{h A 2}\right)\left(\frac{d_{1}}{r_{1}^{2}}-\frac{d_{3}}{r_{3}^{2}}\right)-\left(B_{v A 1}-B_{v A 2}\right)\left(\frac{h}{r_{1}^{2}}-\frac{h}{r_{3}^{2}}\right)\right)+0
\end{gathered}
$$

Equation 2.10 is obtained from Equation 2.7, considering two monitors are symmetric with transmission line $\mathrm{B}$.

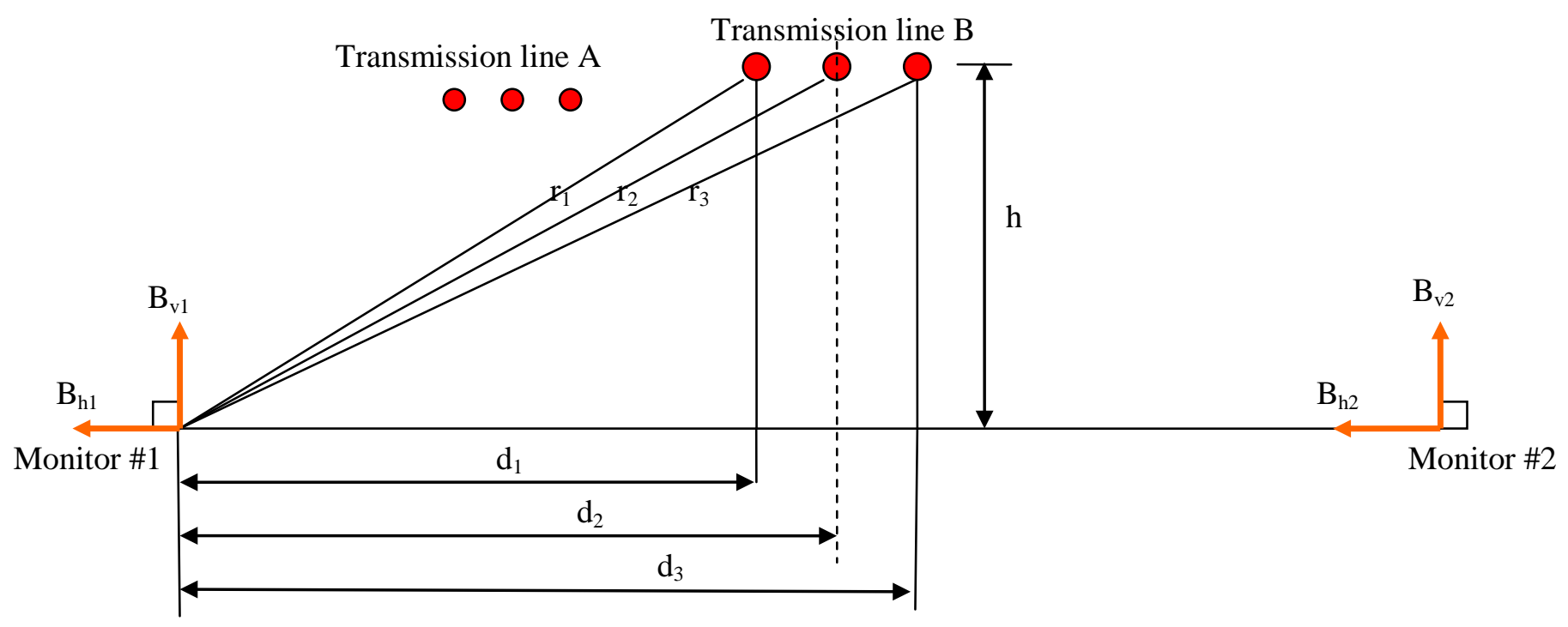

Figure 2-8: Schematic drawing of signal cancellation for parallel horizontal lines 
Theoretically, it is predicted that the influence of one of two transmission lines (in this case Line B) can be cancelled out by putting two monitors symmetric with this line and applying the appropriate calculation (Equation 2. 10).

The validity of this method was tested in the field on a horizontal parallel transmission line set. Figure 2-9 shows sample data obtained from the magnetic field sensors of two monitors placed symmetrically about line B. Figure 2-10 shows the computed cancellation.

Here the cancelled signal is $\left(\left(B_{h B 1}-B_{h B 2}\right)\left(\frac{d_{1}}{r_{1}^{2}}-\frac{d_{3}}{r_{3}^{2}}\right)-\left(B_{v B 1}-B_{v B 2}\right)\left(\frac{h}{r_{1}^{2}}-\frac{h}{r_{3}^{2}}\right)\right)$ and the remaining signal is $\left(\left(B_{h A 1}-B_{h A 2}\right)\left(\frac{d_{1}}{r_{1}^{2}}-\frac{d_{3}}{r_{3}^{2}}\right)-\left(B_{v A 1}-B_{v A 2}\right)\left(\frac{h}{r_{1}^{2}}-\frac{h}{r_{3}^{2}}\right)\right)$. The amplitude of the cancelled signal is non-zero due to measurement error of the magnetic field and the line configuration.

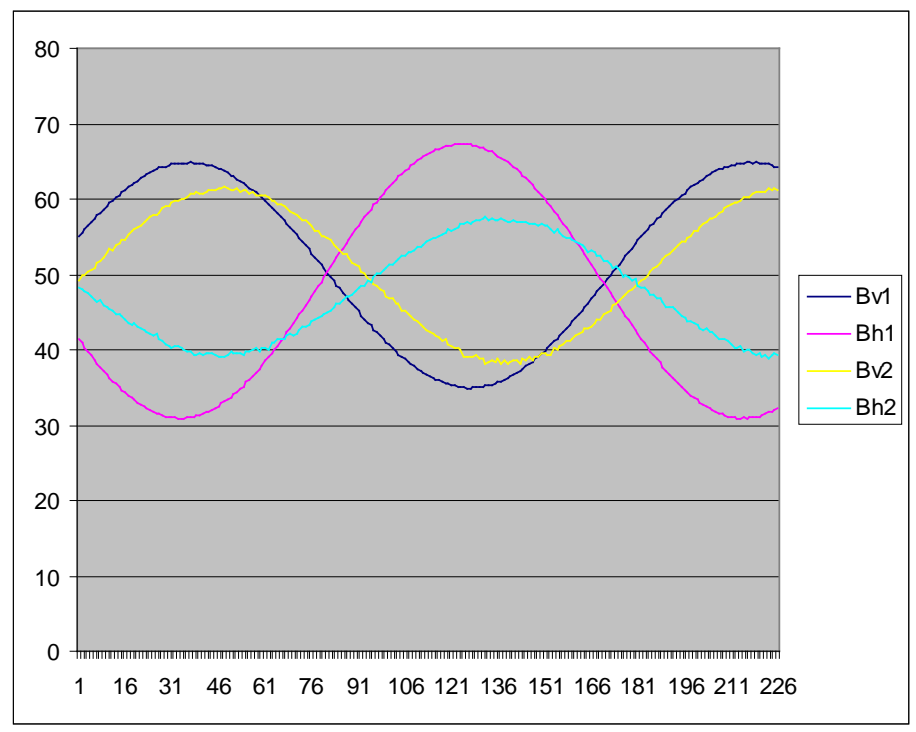

Figure 2-9: Magnetic field measurement by two monitors placed symmetric to line B (Figure 2-8) for a horizontal parallel line set. (Signal amplitude in arbitrary analog-to-digital conversion units) 


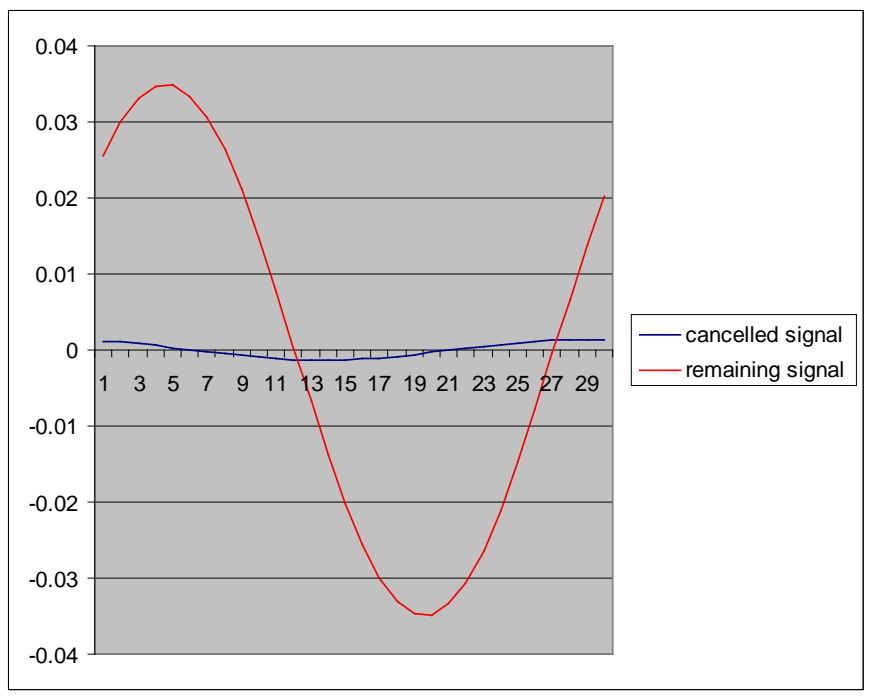

Figure 2-10: The blue line represents the total acquired signal after cancellation. The red line represents the remaining signal representing Line A power flow (arbitrary relative signal values).

\subsection{Multiple Line Sensing - IR Reflectometry}

The current use of a sensing technology based on direct magnetic field measurements is advantageous in that it allows the measurement of signal (i.e. magnetic field strength) which is directly proportional to the power flowing on the line. The disadvantage of direct magnetic field measurement is that it is difficult to isolate magnetic field emanating from an individual line when it is close to other transmission lines and/or other sources of $60 \mathrm{~Hz}$ magnetic field noise.

Transmission lines and power generation equipment undergo low-level mechanical vibrations during normal operation. The frequency spectrum of the vibrations is composed of the ac line frequency along with the mechanical response of the power generating or transmitting structure. The vibrational frequency spectrum can therefore provide information on the conditional status of power generation equipment and electrical transmission lines. Vibro-acoustic methodologies have been used in the past for nonintrusive determination of whether underground electric power cables are energized or de-energized ${ }^{1}$.

Commercial devices ${ }^{10,11}$ which record and analyze transmission line vibration are currently installed by utilities on high voltage transmission lines to study aeolian and other mechanical vibrations on the line. Vibration studies and models have also been suggested as a potential method of non-invasively detecting flaws in equipment such transformers.

In this study we investigate the feasibility of using a non-contact long-range optical reflectometry method to detect mechanically or electrically induced surface vibrations and in addition to probe surface magnetization of the conductor bundles. 
The three hypotheses under investigation were;

1. Reflected light will vary with power flow via a Magneto-Optic Kerr Effect (MOKE). This effect leads to a change in polarization of light from a surface emanating a magnetic field where the degree of polarization change is proportional to the strength of the magnetic field

2. Reflected light will vary due to vibrations on the conductor bundle where the vibration amplitude correlates with the magnitude of power flow.

3. Reflected light will vary with conductor temperature where temperature correlates with the magnitude of power flow.

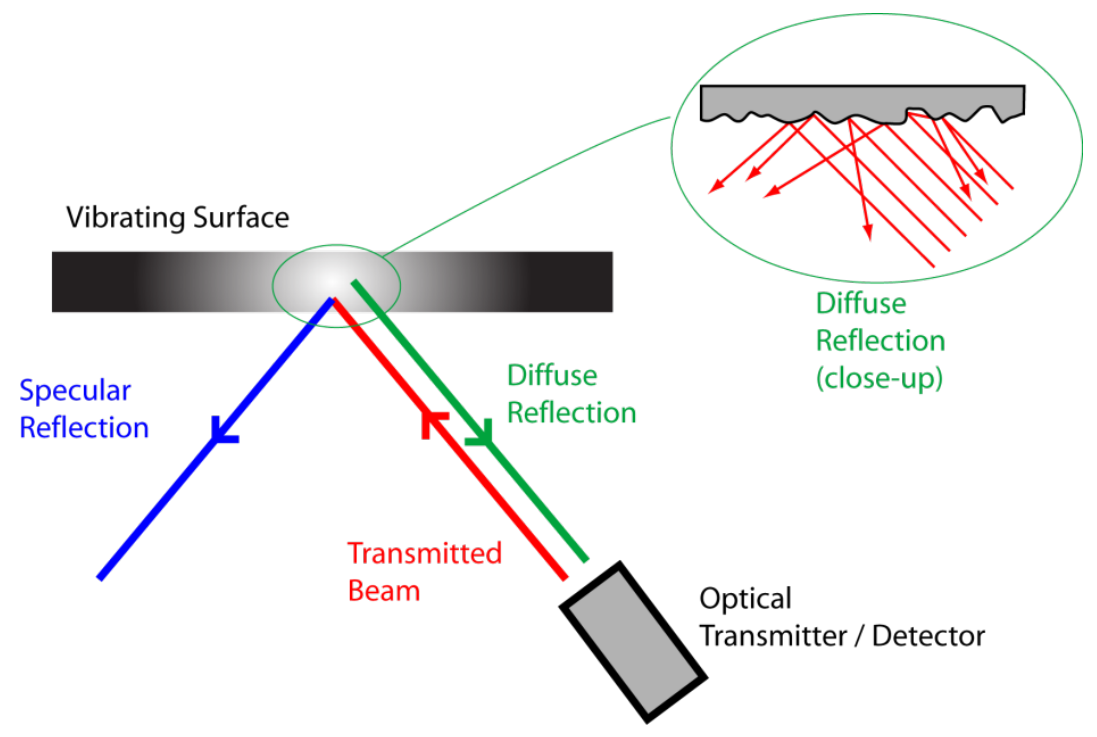

Figure 2-11: Vibro-optical detection scheme

Figure 2-11 shows the concept behind the optical detection of vibrations from a surface. Laser light is directed at the surface by an optical transmitter. The majority of this light undergoes specular reflection, whereby the angle of reflection and transmission to the surface normal are equal. A small percentage undergoes diffuse reflection due to small variations in the surface structure. This light is directed back along the transmitted beam path, and can be detected by a photodiode at the transmission site. Because the amplitude of the diffuse reflected beam is strongly dependent on the surface features, it is also sensitive to vibrations of the surface. The magnitude of the detected signal will thus be modulated at the frequency of the surface vibrations. 
The concept for optical power detection is based on the Kerr effect, in which the polarization of light rotates due to the interaction with a magnetized surface (see Figure 2-12). The magnetic field surrounding a high voltage transmission line is proportional to the current flowing through the line. This should be detectable as a rotation in the polarization of the reflected light as compared with the transmitted light. By monitoring the amplitude of the light detected with a polarizing filter in place, it should be possible to monitor the power flowing through the line.
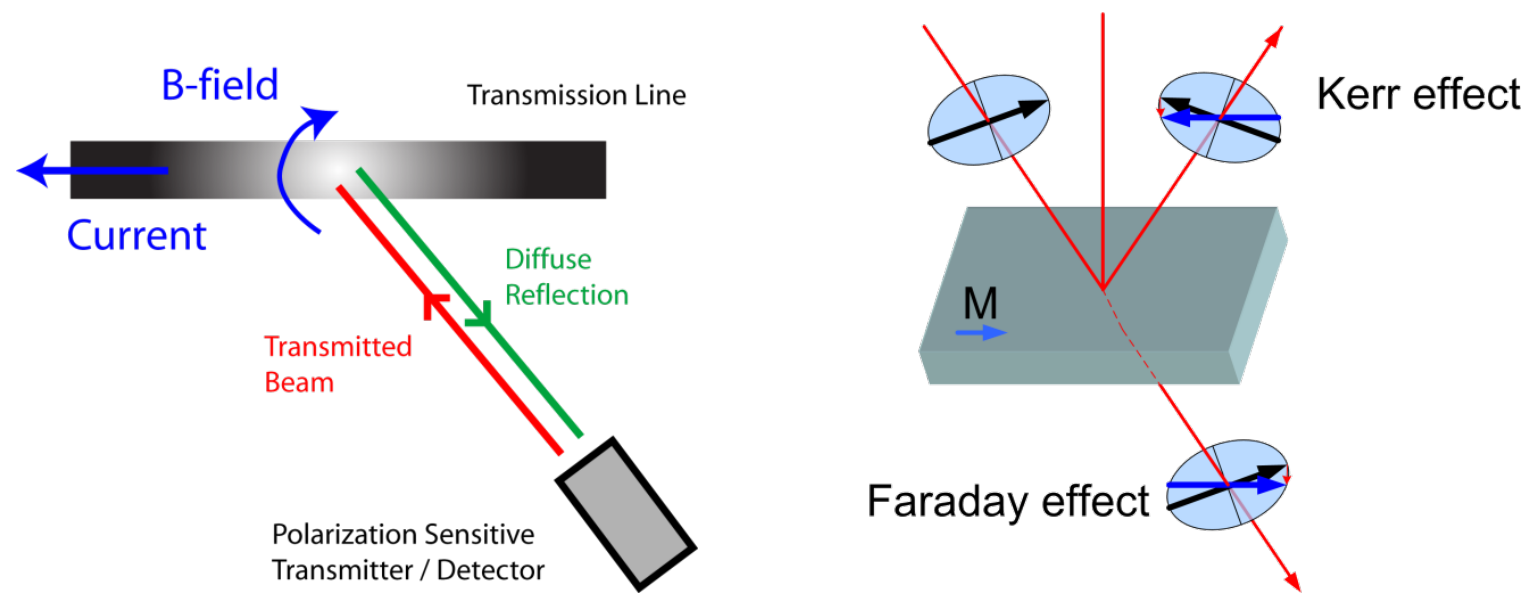

Figure 2-12: Power detection scheme based on Kerr rotation. Faraday rotation describes a similar effect for light transmitted (rather than reflected) through the magnetic medium.

A commercial handheld infrared reflectance device was used to develop a prototype system for collecting reflected optical signals from the surface of overhead conductors in high voltage transmission lines. The device can transmit and detect optical (in the infrared range of the spectrum) signals to distant (maximum typical range $1 \mathrm{~km}$ ) objects. A prototype hand-held optical reflectometry unit was built and tested. The light source is a low-power $1 \mathrm{~mW}$ infrared diode laser. It is driven by capacitive discharge circuit to produce a series of light pulses, each approximately $100 \mathrm{~ns}$ in duration. The standard operating mode of the device involves the emission of a series of 64 light pulses at a repetition rate of $400 \mathrm{~Hz}(2.5 \mathrm{~ms}$ spacing between pulses) for a total probing time of $160 \mathrm{~ms}$. In Phase 1, the device was used in standard operation mode. In Phase 2, the device was modified to allow continuous controlled pulsing.

The device is powered using a standard $9 \mathrm{~V}$ battery. The amplitude of the reflected pulses is detected using a high speed National Instruments (2 G-samples/s) analog-to-digital acquisition card interfaced to a PC. Data acquisition and analysis is performed using a custom written 
Labview software program. This program controls acquisition parameters, outputs raw data and displays a real-time fast Fourier transform (FFT) output of the acquired data. The real-time FFT display allows optimized alignment of the laser beam before data is acquired and saved to the PC disk.

\subsubsection{Phase 1 Laboratory Tests and Analysis}

A Labworks Inc. ET-126B electrodynamic shaker was used as a calibrated vibration source for laboratory testing the vibro-optic device. The shaker table provides frequency bandwidth from DC to $10 \mathrm{kHz}$ and selective velocity up to $3 \mathrm{~m} / \mathrm{sec}$.

Initial measurements on vibrating (shaker table data presented in Figure 2.13) and fixed surfaces (laboratory door and wall data presented in Figure 2.14) revealed significant measurement noise at 80 and $160 \mathrm{~Hz}$. This noise was related to the rapid transmission and sampling of pulse groups required when using the reflectometer device in its standard operating mode. Consecutive groups of 64 pulses were processed using a discrete fourier transform (DFT). The DFTs were then averaged for each acquisition period. The DFT frequency range was $0-200 \mathrm{~Hz}(200 \mathrm{~Hz}=$ $1 /(2 * \mathrm{dt})$ where $\mathrm{dt}$ is $2.5 \mathrm{~ms}$ or the time between pulse groups). The frequency spacing in the DFT is $6.25 \mathrm{~Hz}\left(6.25 \mathrm{~Hz}=1 / \mathrm{N}^{*} \mathrm{dt}\right.$ where $\left.\mathrm{N}=64\right)$. The occurrence of the $160 \mathrm{~Hz}$ noise peak seemed to be related to the frequency spacing, $160 \mathrm{~Hz}=1 /(6.25 \mathrm{~ms})$.

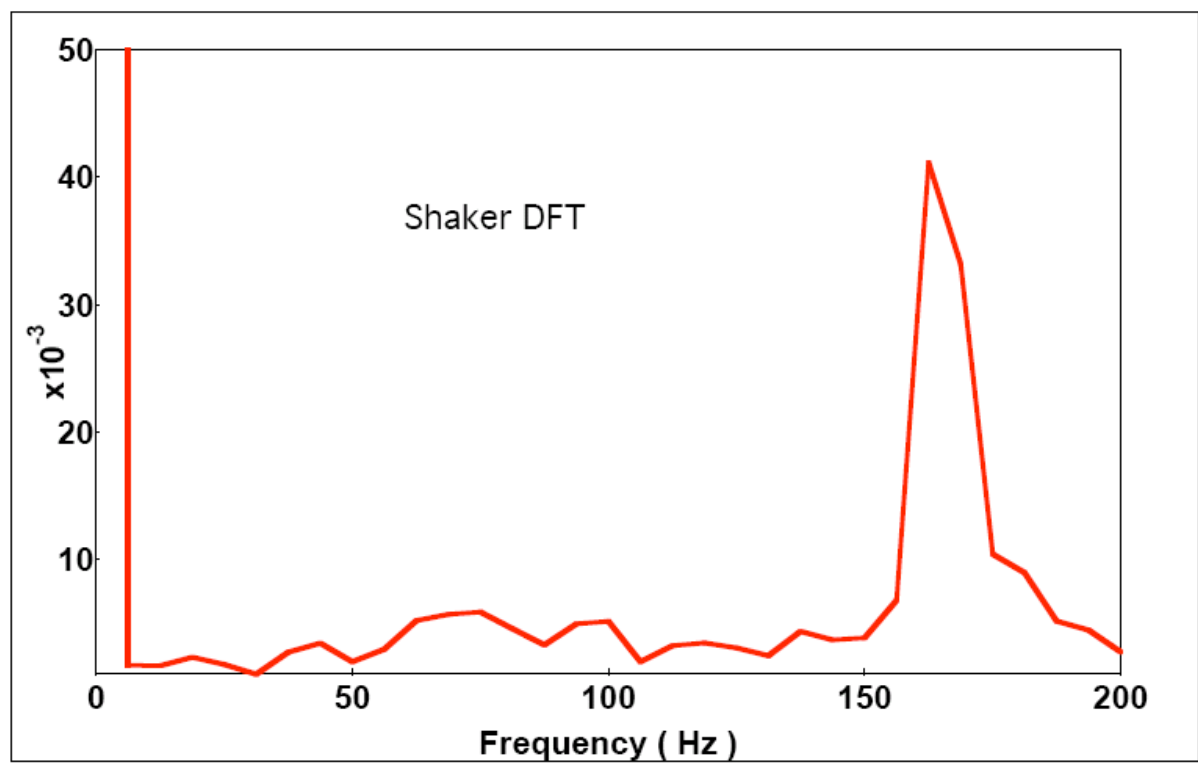


Figure 2-13 DFT spectrum (arbitrary units) acquired from shaker table set to vibrate at $60 \mathrm{~Hz}$. Significant $160 \mathrm{~Hz}$ noise is evident.

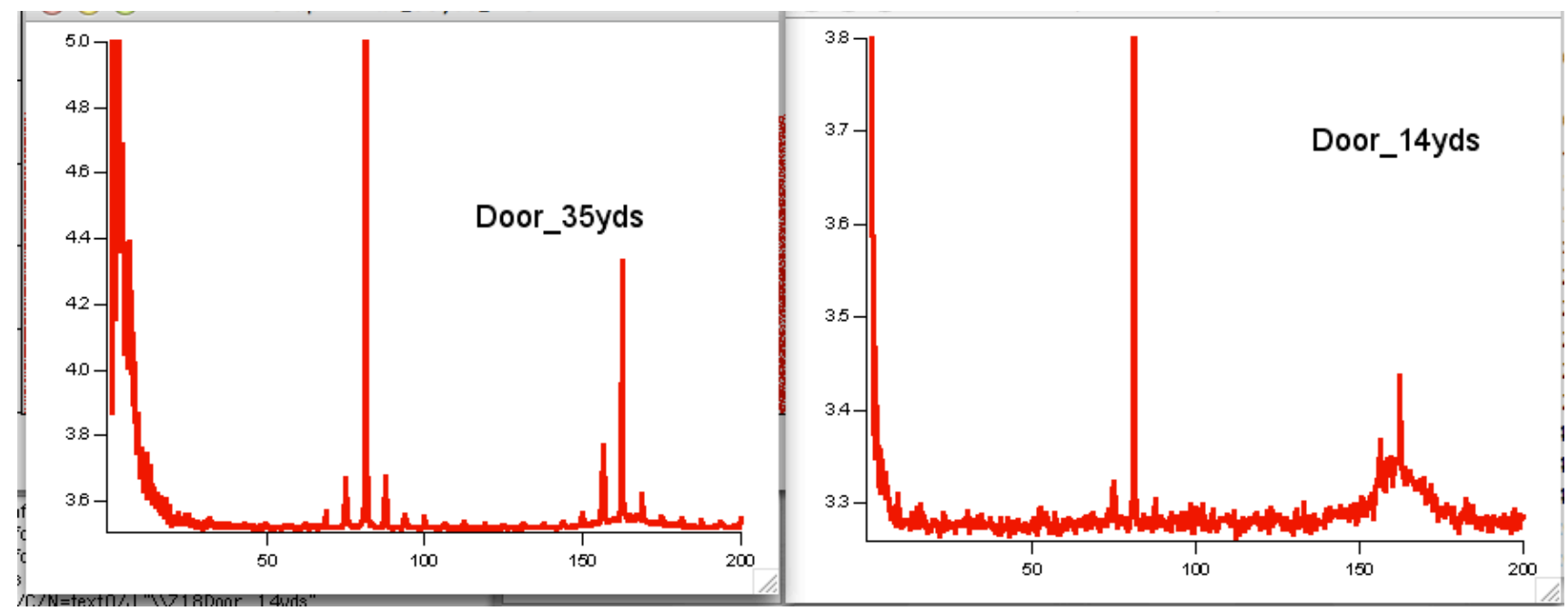

Figure 2-14 FFT spectrum (arbitrary units) acquired from fixed targets (laboratory door and wall)

\subsubsection{Phase 1 Power Line Field Tests and Analysis}

Optical reflection measurements were performed on a number of high voltage overhead transmission lines. Reflected pulses can be detected of distance surfaces up to a distance of 300 $\mathrm{m}$ in ambient daylight.

\section{Case 1: Current Flow Compared to No Current Flow}

Two lines $(345 \mathrm{kV}, 138 \mathrm{kV})$ were tested that run along the same right-of-way. During testing, the $345 \mathrm{kV}$ line (referred to as front line in Figure 2.16) was energized, but was not carrying any current. The $138 \mathrm{kV}$ line (referred to as rear line in Figure 2.16) was energized and carrying current. During tests, data was acquired from the reflectometer device with and without the 
polarizing filter installed which detects rotations in the polarization of the light. The wooden pole supporting the $138 \mathrm{kV}$ line was used as a control independent of current flow in the experiments.

The wooden pole was chosen as a control surface in preference to the ground wire due to the difficulty of focusing the optical beam onto the narrow surface of the ground wire at distance. Measurements were acquired from the energized and powered $138 \mathrm{kV}$ line from two positions 1 , 2 as shown in Figure 2.15.

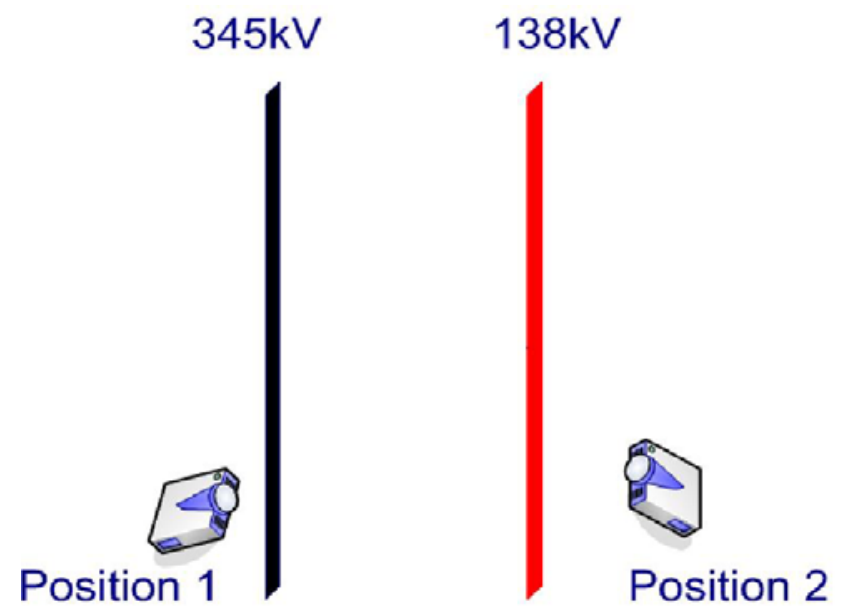

Figure 2-15 Reflectometer measurement positions relative to two parallel lines ( $345 \mathrm{kV}$ and $138 \mathrm{kV}$ ) at the test site.

Observations:

Although limited in reproducibility, some evidence for fundamental power line vibrations due to current flow (60 and $120 \mathrm{~Hz}$ peak) were observed in one set of experiments using the polarizer Figure 2.16 (centre right, blue plot) on the powered line only.

Measurements in general were noisy, especially when taken at position 1 (near the $345 \mathrm{kV}$ line). Measurements at position 2 showed significant improvement in signal-to-noise and general reproducibility. 80 and $160 \mathrm{~Hz}$ peaks appear strongly on the control surface (wooden transmission line mounting pole) similar to that seen in laboratory experiments. 
Spectral content was measured which appeared unique to the line measurements and which were not observed in laboratory tests. A peak at approximately $110 \mathrm{~Hz}$ appeared in most of the measurements on both the powered and unpowered lines.

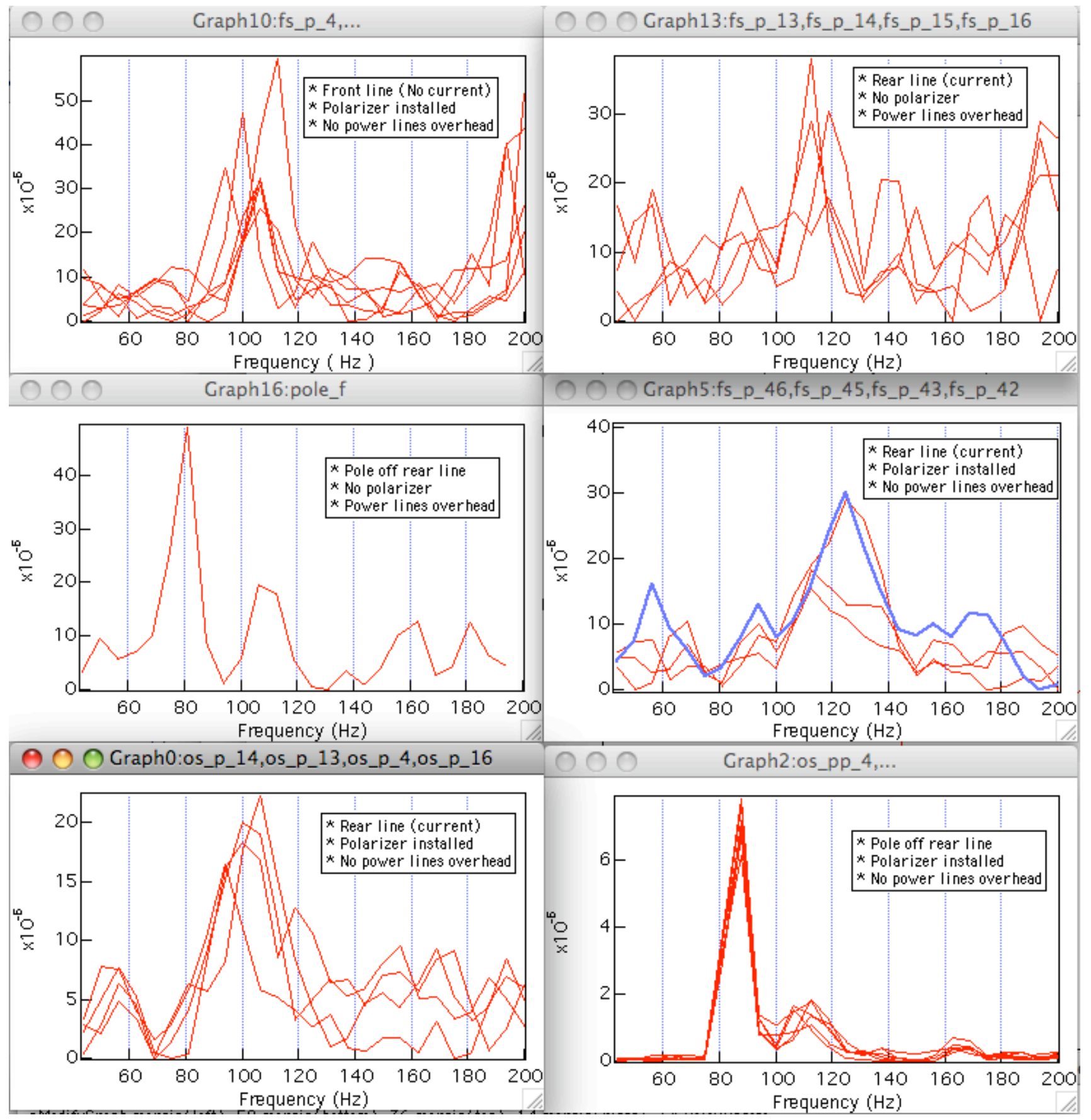


Figure 2-16 The plots are arranged in the order in which data was acquired. Top Left: measurements of the front line with no current Top Right: measurements of the rear line (taken at position 1), Central Left: control measurements from wooden mounting pole with polarizer installed Central Right: measurements of the rear line (taken at position 2) Bottom Left: repeat measurement of the rear line (taken at position 2) Bottom Right: control measurements from wooden mounting pole with no polarizer installed

\subsubsection{Phase 2 Reflectometer Signal-to-Noise Optimization}

In Phase 1, it was discovered that the small number of pulses within each individual cycle and the short probing time of the reflectometer device in its standard operating mode meant that peaks in the Fourier spectrum were very broad, with poor resolution and poor signal to noise ratio.

In order to improve overall signal-to-noise for the reflectometer device and alleviate signal processing complexities, a number of improvements were carried out in a Phase 2 of the development effort. A programmable microcontroller was interfaced with the optical pulse generation circuit of the device. An emission control circuit allows a $100 \mathrm{~ns}$ optical pulse to be emitted at a repetition rate of up to $10 \mathrm{kHz}$, for a probing time of up to $1 \mathrm{~s}$ for a total of 10,000 pulses per measurement series.

The microcontroller can also be used to bias the detector circuit so that the reflected pulses are detected at maximum sensitivity.

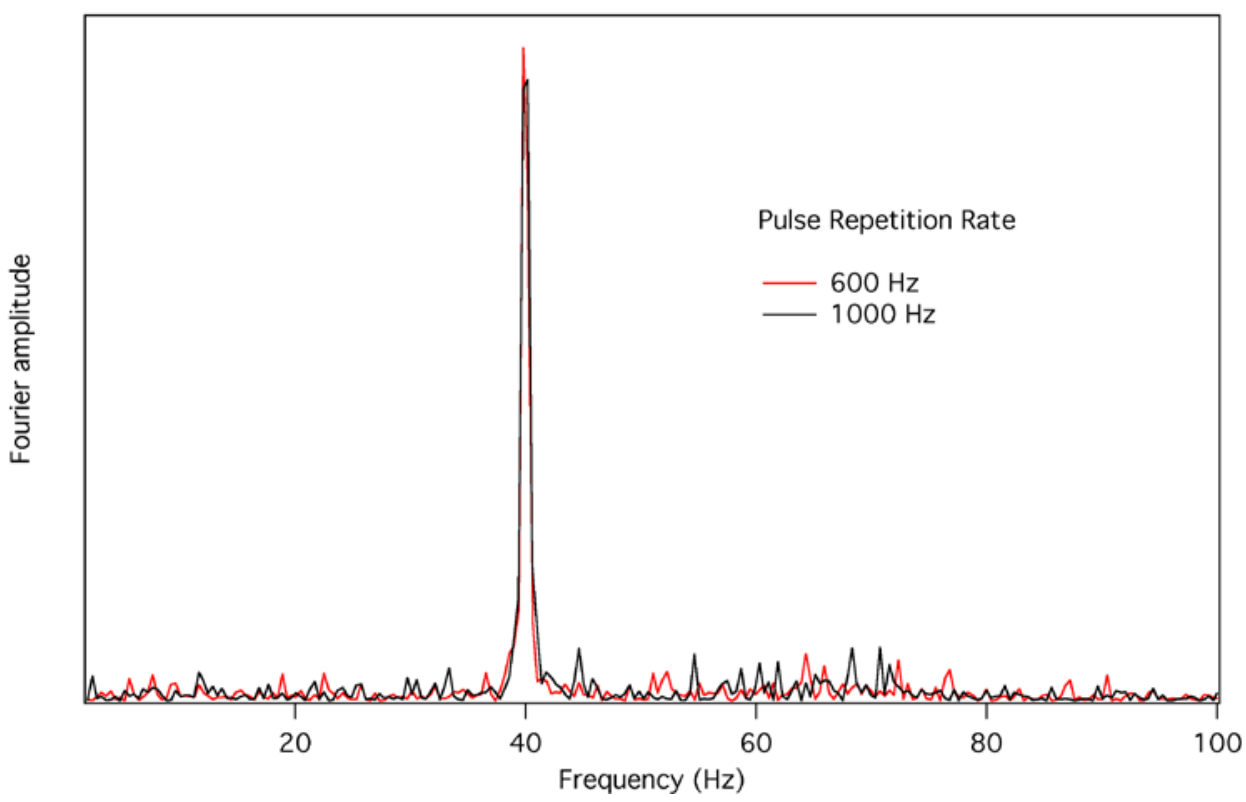

Figure 2-17 Power spectrum (arbitrary units) of the light amplitude reflected from a shaker table vibrating at $40 \mathrm{~Hz}$ using optimized reflectometer device. 
Laboratory tests show that these changes have resulted in a vast improvement in the detection sensitivity. The 80/160 Hz noise peaks are no longer evident. Figure 2.17 shows the Fourier transform of the pulse amplitude reflected from a shaker table vibrating at $40 \mathrm{~Hz}$. A clear peak at $40 \mathrm{~Hz}$ is observed in the reflected light pulse, with excellent signal discrimination. Both 600 $\mathrm{Hz}$ and $1 \mathrm{kHz}$ repetition rates produce similar results. Future work will concentrate on further power line field studies with this optimized device.

\section{Prototype Development of Power Scavengers and Directional Sensors}

\subsection{Prototype Development of Power Scavengers}

\section{Vibration Scavenging - Prototype Design and Field Testing}

Measurements from various environmental vibration sources were conducted in the downtown area of Louisville, Kentucky. A busy urban bridge which runs over the Ohio River was chosen for testing. Measurements were conducted at various times for vibrations associated with a free standing streetlight pole. The experimental set-up is shown in Figure 3-1. The scavenging device prototype was strapped to the bridge-light pole. The device shown in Figure 3-2 is light and portable. In the first set of field tests, the voltage output of the PZT cantilever is measured directly. Figure 3-3 shows a sample voltage output for the prototype scavenging device.

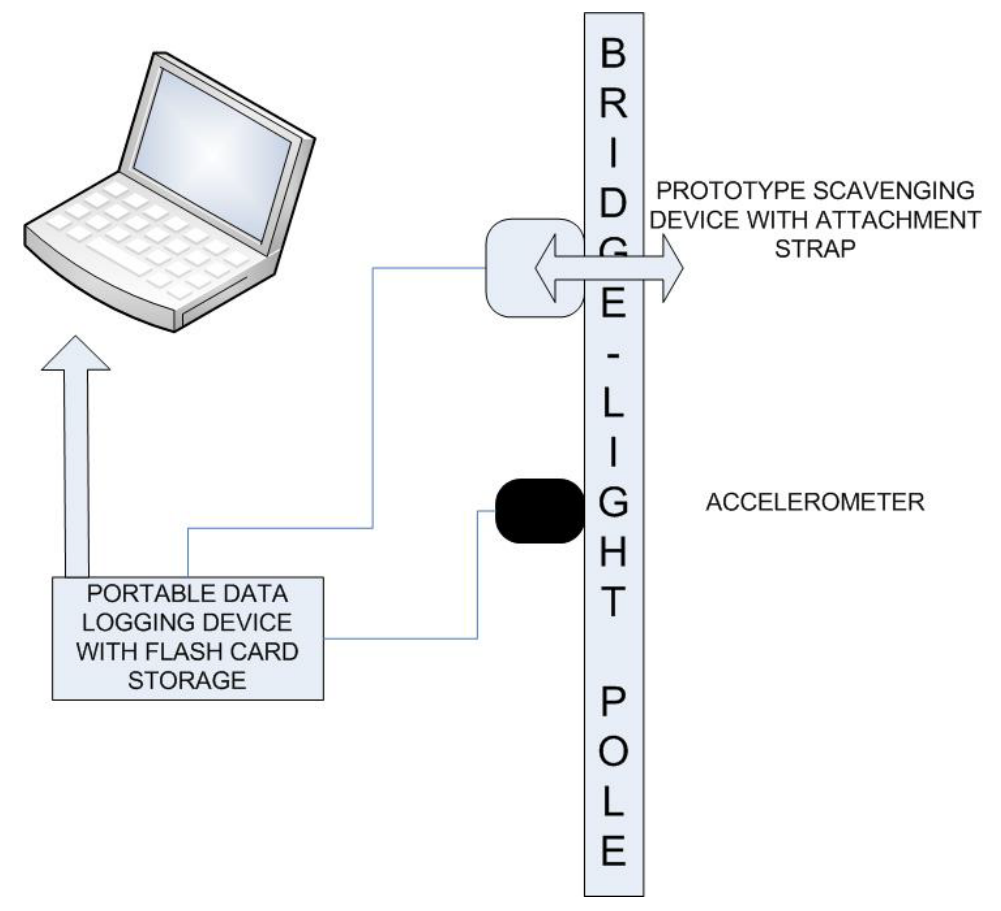


Figure 3-1: Schematic showing simultaneous data acquisition of vibrations (using an accelerometer) and voltage output (from the vibration scavenging prototype) from a light pole on the bridge. Data acquisition is performed using a portable battery powered data logging device which stores data on a flash card interface for download to a PC for data analysis.

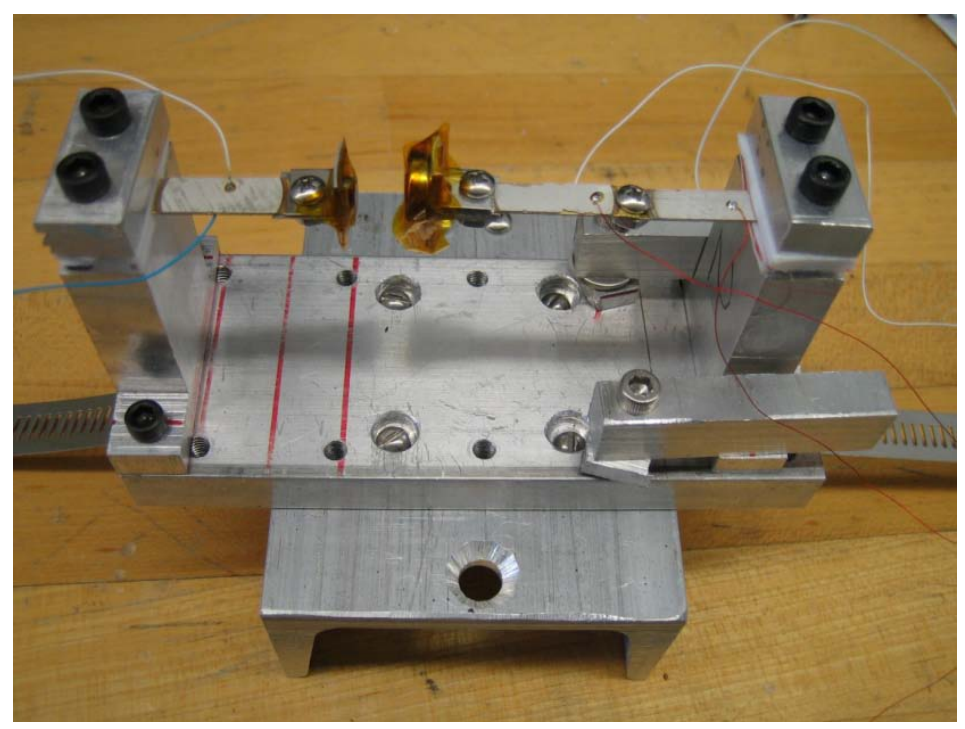

Figure 3-2: Photo showing the vibration scavenging prototype. The device is mounted on a flexible strap for mounting to a pole.

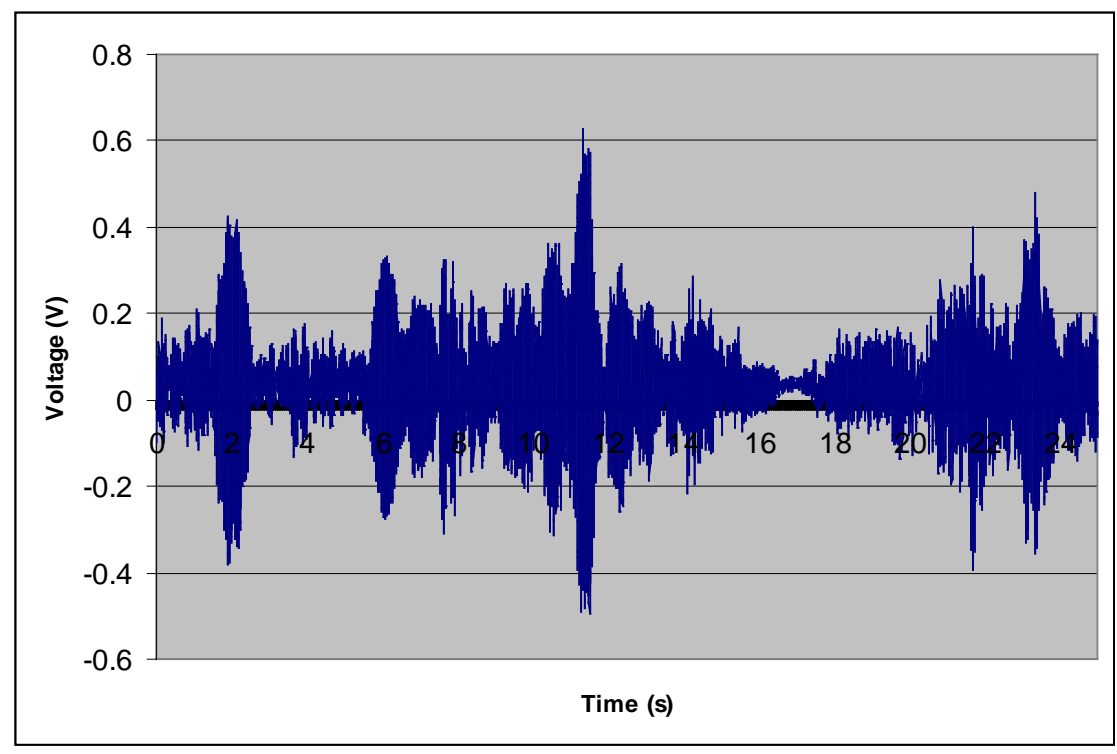

Figure 3-3: Voltage output from prototype scavenging device attached to a streetlight pole on a busy urban bridge. Output levels depend on bridge traffic with peaks associated with the passing of large vehicles such as trucks. 


\subsection{Prototype Development of Directional Sensors}

Two 3-axis magnetometer sensors (Honeywell HMC2003, MicroMag3) were compared to a commercial 3-axis magnetometer system in side-by-side field tests as potential candidates for integration into the power line monitor. The sensors was interfaced to mobile data acquisition units for field testing at the DC line testing facility at Oakridge National Laboratory in Oakridge, Tennessee. An AC line test facility would have been preferred for the work, however there is no known AC power line testing facility available for commercial testing in the US.

\subsubsection{Prototype and Commercial Calibration Systems}

\section{Honeywell HMC2003}

This chip magnetometer is a high sensitivity, three axis magnetic sensor hybrid assembly used to measure low magnetic field strengths ${ }^{12}$. The features of this sensor are listed below in Table 3-1.

\section{MicroMag3}

The MicroMag3 is an integrated 3-axis magnetic field sensing module that measures the earth's magnetic field and the magnetic field produced by high voltage power lines. Features of the MicroMag3 are listed in Table 3-2. 


\begin{tabular}{|l|}
\hline Features of Honeywell Sensor \\
\hline 20-pin Wide DIP Footprint (1" by $\left.0.75^{\prime \prime}\right)$ \\
\hline Precision 3-axis Capability \\
\hline Factory Calibrated Analog Outputs \\
\hline 40 micro-gauss to \pm 2 gauss Dynamic Range \\
\hline Analog Output at 1 Volt/gauss (2.5V @ 0 gauss) \\
\hline Onboard +2.5 Volt Reference \\
\hline 6 to +15 Volt DC Single Supply Operation \\
\hline Very Low Magnetic Material Content \\
\hline Neg $40^{\circ}$ to $85^{\circ} \mathrm{C}$ Operating Temperature Range \\
\hline
\end{tabular}

Table 3-1 Features of Honeywell sensor [12]

\begin{tabular}{|l|}
\hline Features of MicroMag3 \\
\hline Complete 3-axis magnetometer draws $<500 \mu \mathrm{A}$ at 3 \\
VDC. \\
\hline Operation as low as 2.2 VDC. \\
\hline Part size: $25.44 \times 25.1 \times 19 \mathrm{~mm}$. \\
\hline DIP configuration for easy prototyping. \\
\hline Field measurement range $\pm 1100 \mu \mathrm{T}( \pm 11$ Gauss) \\
\hline Resolution as low as $0.015 \mu \mathrm{T}(0.15 \mathrm{mG})$ \\
\hline All digital solution, no additional circuitry is required. \\
\hline
\end{tabular}

Table 3-2 Features of MicroMag3 [13]

\section{Bartington Mag-03}

The Bartington Mag- $03^{13}$ low noise sensor and the Spectromag-6 device is a field portable magnetic field measuring system. The Mag-03 sensor is a 3-axis high performance fluxgate sensor with integral electronics to measure either static or alternating fields. The sensor used in these tests has a 70 microtesla range and a bandwidth up to $3 \mathrm{kHz}$. The Spectromag-6 is a 6 channel spectrum analyzer that is capable of simultaneously measuring a combination of magnetometers, accelerometers, and/or microphones. The system displays both frequency and time domain plots and is useful for varying test parameters to obtain the desired information [1]. Features of both instruments are listed in detail in Table 3-3 and 3-4 below. 


\begin{tabular}{|l|l|}
\hline \multicolumn{2}{|l|}{ Features of Mag-3 Magnetic Sensor } \\
\hline Supply Voltage & $\pm 12 \mathrm{~V}$ \\
\hline Frequency Response & 0 to $1 \mathrm{kHz}$ maximally flat \\
\hline Calibration Error & $\pm 0.5 \%$ \\
\hline Bandwidth & 0 to $3 \mathrm{kHz}$ \\
\hline Internal noise & $<6 \mathrm{pTrms} / \sqrt{\mathrm{Hz}}$ at $1 \mathrm{~Hz}$ \\
\hline Supply Current & $+26 \mathrm{~mA},-6 \mathrm{~mA}$ \\
\hline Measuring Range & $\pm 70 \mu \mathrm{T}$ \\
\hline Offset error & $\pm 5 \mathrm{nT}$ \\
\hline & \\
\hline
\end{tabular}

Table 3-3 Features of Mag-3 [2]

\begin{tabular}{|l|}
\hline Features of SpectroMag-6 \\
\hline 6 -channel, simultaneously sampled, 24 bit data \\
acquisition \\
\hline Magnetic Field and Vibration measurement inputs \\
\hline Time domain and Frequency domain display, with zoom \\
facility \\
\hline $100 \mu s$ to 10 s sample intervals \\
\hline Fixed scan length or continuous acquisition mode \\
\hline Direct connection of ICP accelerometers or microphones \\
\hline Operates from mains power or internal, rechargeable \\
battery \\
\hline
\end{tabular}

Table 3-4 Features of SpectroMag-6 [2]

\subsubsection{Test Procedure}

In order to insure correct orientation of the instruments, commercial survey equipment was used for leveling with respect to the longitudinal axis of the power line. A survey procedure was developed $^{14}$ in order to achieve reproducible positioning with respect to the power line. As shown in Figure 3.4, a wooden structure was built to attach all three sensors to the survey equipment for correct alignment and positioning.

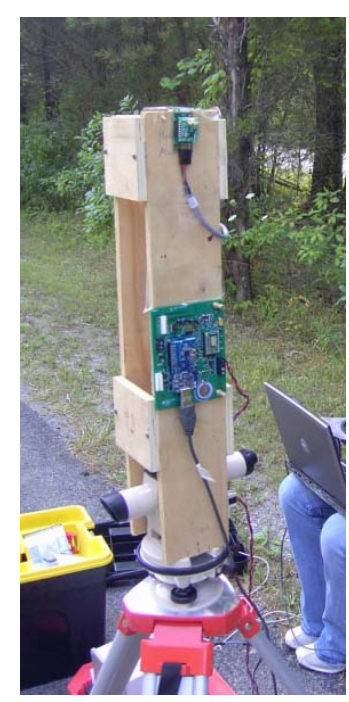


Figure 3-4 Sensor alignment with respect to the line was achieved with a wooden mounting structure in combination with the survey level.

\subsubsection{Oakridge DC Powerline Test Facility}

The DC Powerline Test Facility is described in http://www.oe.energy.gov/DocumentsandMedia/Power_Line_Conductor_Accelerated_Testi ng-Stovall.pdf and shown in Figure 3.5. The DC power line can be ramped, lowered at specific rates or held at a static current level. Figure 3.6 shows the operation of the line during the test period. Data was measured at two positions: close to the line (approximately 9 feet) and away from the line (approximately 35 feet).

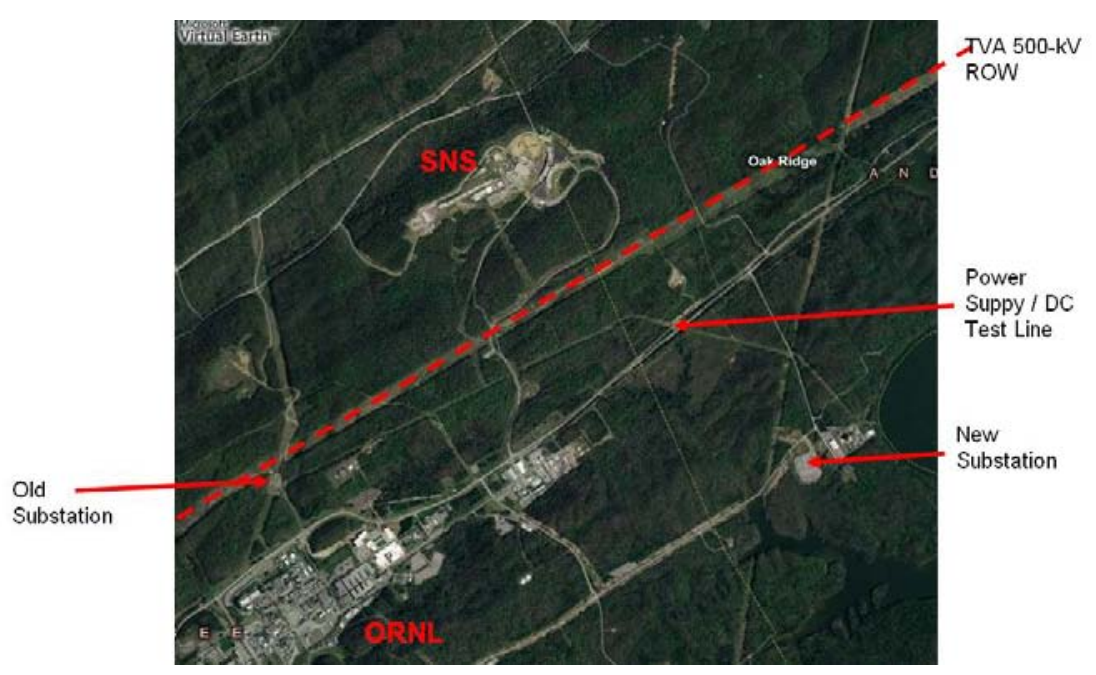

Figure 3-5 ORNL DC Test Site 


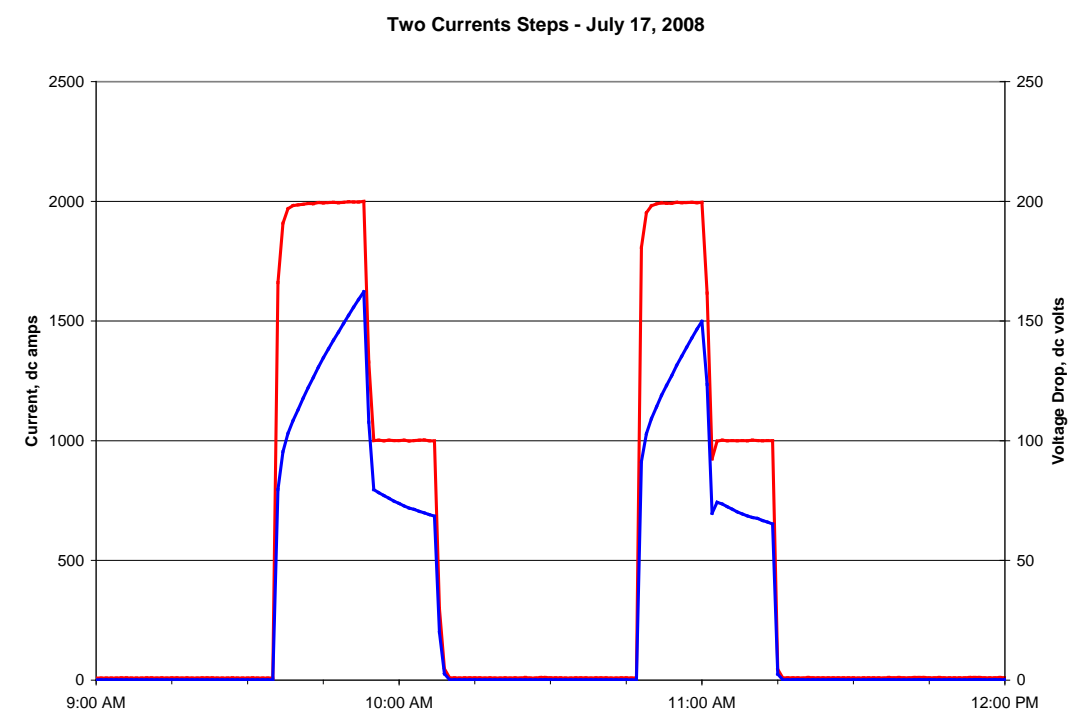

Figure 3-6: Controlled DC line current ramp on and ramp off transitions during testing

At each distance the magnetic field strength (with a 1000A and a 2000A current traveling through the line) and the background magnetic field noise was recorded with each sensor.

Three reading from each sensor was recorded and averaged. Figure 3.7 shows the results from all three sensors. The signal strength is the direct output from the sensors. The values are not calibrated to a known magnetic field strength.

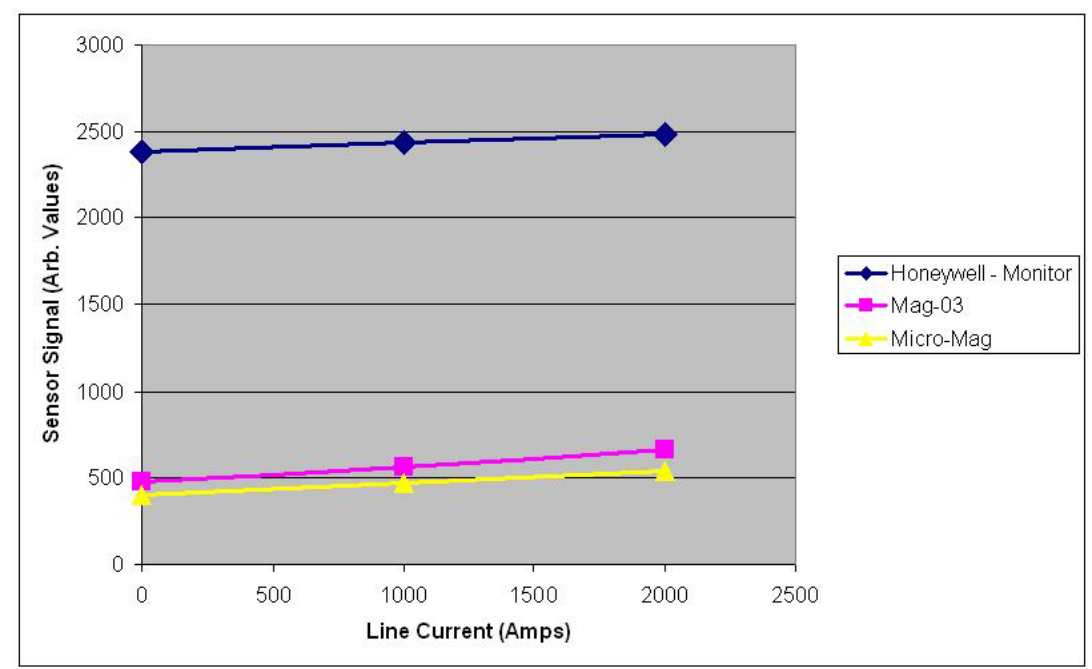

Figure 3-7 Comparison of three commercial 3-axis sensors (Mag-03, Micro-Mag, Honeywell) 
The Biot-Savart equation was used to find the theoretical magnetic field strength in the $\mathrm{x}$ and $\mathrm{y}$ directions at each location.

$$
B=K^{\prime} \frac{2 I}{d}
$$

In equation 3.1, B is the magnetic field strength without any background noise, $\mathrm{K}$ ' is a constant equal to $10^{\wedge}(-7) \mathrm{N} / \mathrm{A}^{\wedge} 2$, I is the current in Amps, and $\mathrm{d}$ is the distance from the sensor to the wire. Since a DC power line has two lines with current traveling in opposite directions, the BiotSavart equation was applied to each line and the $\mathrm{x}$ and $\mathrm{y}$ components were added together to arrive at the final theoretical values. From the results presented, the theoretical values closely relate to the experimental values except for the case of the By values. The reasons for this are not clear and would require further testing to investigate.

The graphs below show the theoretical change in the magnetic field strength components due to distance from the DC lines. It also displays the actual change measured by the Mag-03 at specific locations. The Mag-3 system is calibrated to output magnetic field strength in units of milligauss.

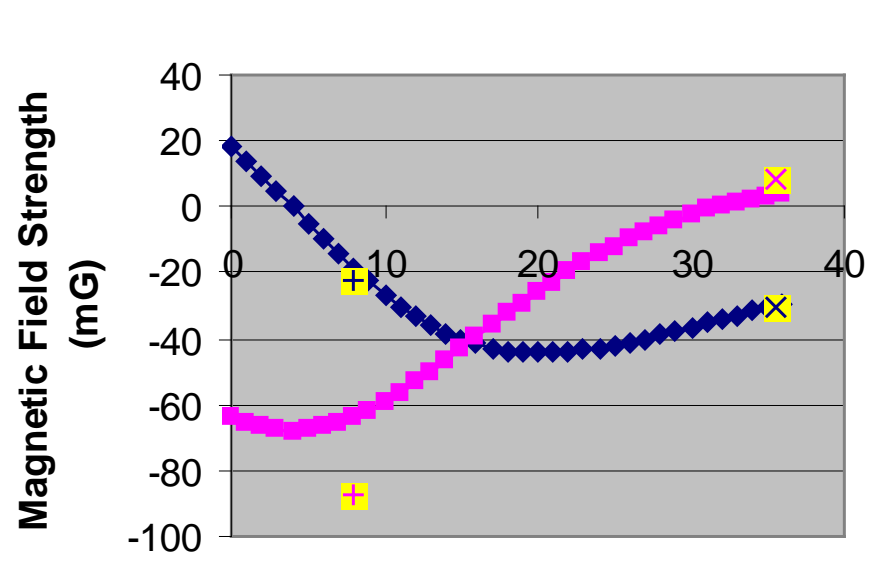

(ft)

Figure 3-8 Theoretical and actual magnetic field strengths with a $1000 \mathrm{~A}$ current flow. $\mathrm{Bx}$ is the horizontal component of the magnetic field, By is the vertical component of the magnetic field, blue cross 
is $\mathrm{Bx}$ at a position 9 feet from the line, pink cross is By at a position 9 feet from the line, blue $\mathrm{X}$ is $\mathrm{Bx}$ at a position 35 feet from the line, pink $\mathrm{X}$ is By at a position 35 feet from the line.

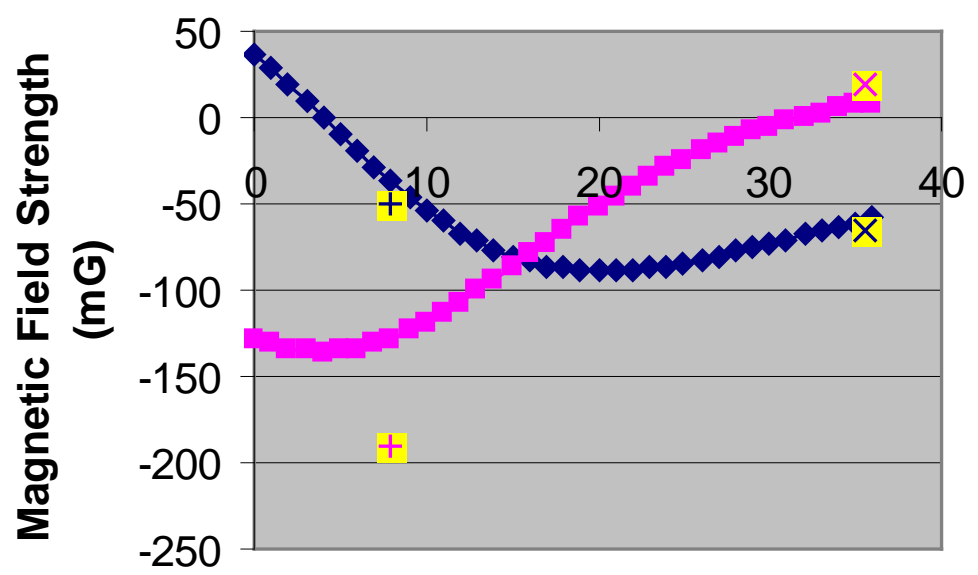

(ft)

Figure 3-9 Theoretical and actual magnetic field strengths with a 2000A current flow. Bx is the horizontal component of the magnetic field, By is the vertical component of the magnetic field, blue cross is $\mathrm{Bx}$ at a position 9 feet from the line, pink cross is By at a position 9 feet from the line, blue $\mathrm{X}$ is $\mathrm{Bx}$ at a position 35 feet from the line, pink $\mathrm{X}$ is By at a position 35 feet from the line.

\subsubsection{Conclusion}

The Honeywell HMC2003 compared well with the commercial systems in field tests at the DC line testing facility when compared to the commercial Mag-3 system. Based on this work the Honeywell HMC2003 was chosen as the candidate for integration into the power line monitor as the 3-axis sensor component. Since there are a number of critical DC transmission lines on the US grid, the feasibility of measuring DC lines such as those tested here is of additional interest in grid monitoring applications. 


\section{Integration Activities Including Form Factor / Function / Cost Optimization}

\subsection{Phase 1 - Low power optimization}

A power requirements analysis was completed for various components of the proposed transmission line monitor including the microprocessor, communication modem and sensors as presented in Table 4.1. The goal was to reduce the frequency of situations that require the components to "operate" thereby reduce overall power consumption. Lower and optimized power requirements for the power line monitor will make the power scavenging methodology presented above more feasible.

Table 4-1: Proposed power requirements and sources for transmission line monitor

\begin{tabular}{|c|c|c||c|}
\hline $\begin{array}{c}\text { Monitor } \\
\text { Component }\end{array}$ & Current & Proposed & $\begin{array}{c}\text { Potential Power } \\
\text { Sources }\end{array}$ \\
\hline $\begin{array}{c}\text { Communication } \\
\text { Modem and Antenna }\end{array}$ & $3 \mathrm{~W}(12 \mathrm{~V})$ & $0.6 \mathrm{~W}(5 \mathrm{~V})$ & $\begin{array}{c}\text { Solar, Permanent } \\
\text { Battery }\end{array}$ \\
\hline Microprocessor & $\begin{array}{c}660 \mathrm{~mW}(12 \mathrm{~V})- \\
\text { processing mode }\end{array}$ & $\begin{array}{c}10 \mathrm{~mW}(3.3 \mathrm{~V})- \\
\text { processing mode }\end{array}$ & $\begin{array}{c}\text { Vibration, } \\
\text { Magnetic/Electric } \\
\text { Field Scavenging }\end{array}$ \\
\hline $\begin{array}{c}\text { Mlectric Field Sensor } \\
\text { Sensor }\end{array}$ & Capacitive plate & $\begin{array}{c}\text { Smaller form factor } \\
\text { capacitive plate }\end{array}$ & Passive \\
\hline Battery Source & $12 \mathrm{~V}$ & $\begin{array}{c}\text { Solid State Sensor } \\
120 \mathrm{~mW}(6 \mathrm{~V})\end{array}$ & Vibration \\
\hline
\end{tabular}

Using the low power Crossbow Mote processor and lower power consumption modem, the proposed power model suggests that the current 12 Volt marine battery which measures $4 * 5 * 2$ inches and weighs 5.5 pounds can be replaced in the novel transmission line monitor by a $5 \mathrm{~V}$ Lithium battery measuring $1.3 * 1.9 * 0.2$ inches and weighing less than 1 ounce as shown in Figure 4.1. 


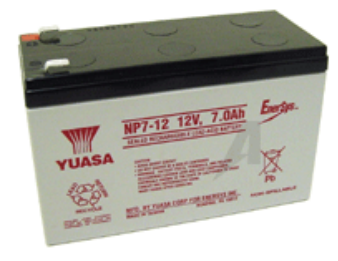

LEAD ACID 12 Volt 7.0AH

Dimensions: 5.94 x 2.56 inches

Height 3.70 inches

Weight 5.5 lbs

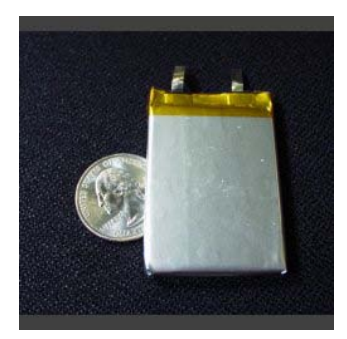

LITHIUM ION POLYMER

Dimensions: $0.23 \times 1.32 \times 1.86$ inches Weight: $0.77 \mathrm{oz}$

Figure 4-1: Current and proposed battery size and weight comparison

\subsubsection{Processor implementation using low power Crossbow Mote platform}

The Crossbow Mote TelosB Mote Research Platform (TPR2400CA) was chosen as the processor platform for the transmission line monitor for the following reasons;

- Low power

- Low cost

- Small size

- Capable of mote-to-mote communication via ZigBee compliant radios (This capability for mote-to-mote communication is advantageous for handheld interrogation of data from the transmission line monitor or in designing networked sensor arrays for directional line sensing in high line density environments.)

The mote processor interface hardware was designed, developed and tested as part of this project, and interfaced to existing sensors and power sources to demonstrate side-by-side comparison of existing commercial Genscape monitor processors. 


\subsubsection{Data Transmission}

The existing commercial transmission line monitor utilizes an analog cellular technology known as AMPS (Advanced Mobile Phone System) in combination with a dedicated machine data network service provided by Aeris (www.aeris.net). The AMPS modem draws 3W of power in communication mode. Aeris recently introduced a newer generation digital cellular technology, known as Code Division Multiple Access (CDMA). The equivalent CDMA modem consumes $0.6 \mathrm{~W}$ of power in communication mode and was put forward as the lower power communication hardware solution in this project.

A development test interface was written to perform initial tests with the CDMA modem. Simple messaging capability was demonstrated using a prototype interface to the Mote processor via the CDMA network. A modern interface printed circuit board was designed and manufactured for the Mote processor system, and a prototype transmission line monitor utilizing an integrated Mote processor-CDMA modem combination was developed for side-by-side field testing using the existing Genscape transmission line monitor as a source of data reference.

\subsection{Phase 2 - New Sensor Integration}

A high sensitivity, three-axis magnetic hybrid assembly Honeywell (HMC2003 Magnetic Hybrid) shown in Figure 4.2 was chosen to perform directional sensor research based on 3-axis magnetic field measurements described in Section 3. A prototype sensor using this chip was developed and interfaced with the Crossbow Mote processor for simple proof-of concept laboratory testing. A data acquisition and viewing application was developed to view and analyze the data acquired from the 3-axis magnetic field sensor by the Mote (Figure 4-3).Tests were performed comparing the output of the 3-axis chip with the output of the 2-axis solenoidbased magnetic field sensors under controlled magnetic field conditions. Measured results between the 3-axis chip and solenoid-based magnetometers used in current power line monitors agree to within $+/-3 \%$. 


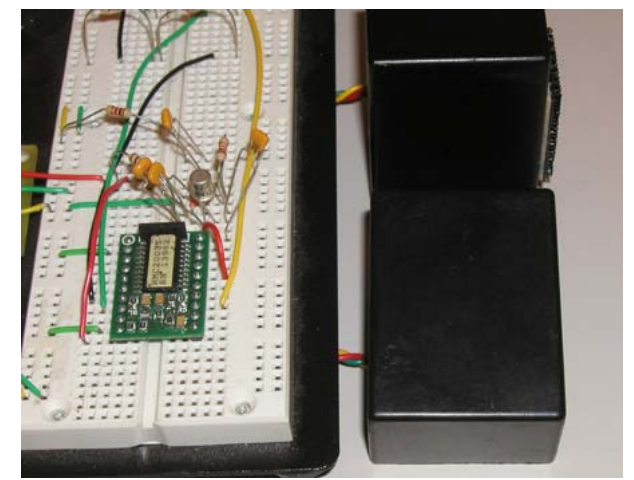

Figure 4-2: 3-axis chip (mounted on breadboard) shown with two solenoid-based magnetometers (enclosed in black casing)

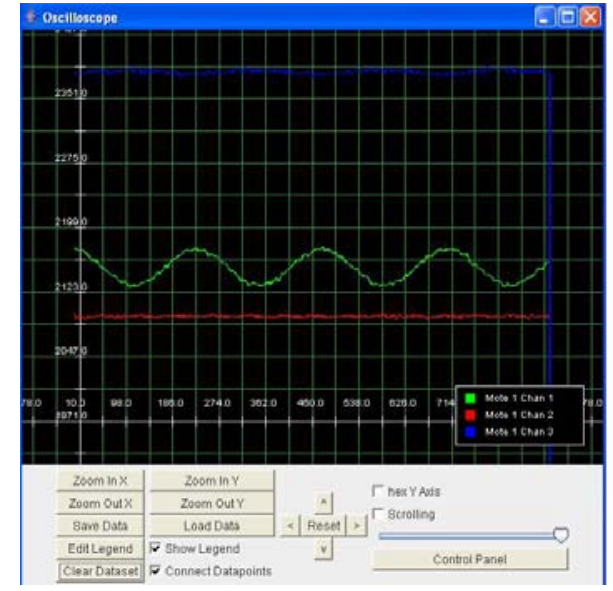

Figure 4-3: Graphical user interface to view and analyze the data acquired by the Mote processor from the 3-axis magnetic field sensor

\section{Phase 3 - Integration of 3-axis Sensor}

Figure 4.4 shows an overview of the integration activities required for prototype development of the power line monitor. First, the processor in the original line monitoring system was replaced by the Mote processor, to create Prototype 1. Next, second and third generation prototypes were assembled using two parallel development paths. Prototype 2 involved the integration of a newer cellular technology (CDMA) modem for use with the Mote processor. Prototype 3 (shown in Figure 4.5) involved the integration of the 3-axis magnetic field sensor. Both Prototype 2 and 3 were then field tested

Figure 4.6 shows an overview of Prototype 4 development, which contains the 3-axis sensor, the cellular modem technology and the mote processor integrated into one package. Work then proceeded to Prototype 5 (Figure 4.7) in which the large $12 \mathrm{~V}$ battery was replaced by a small lithium ion battery 


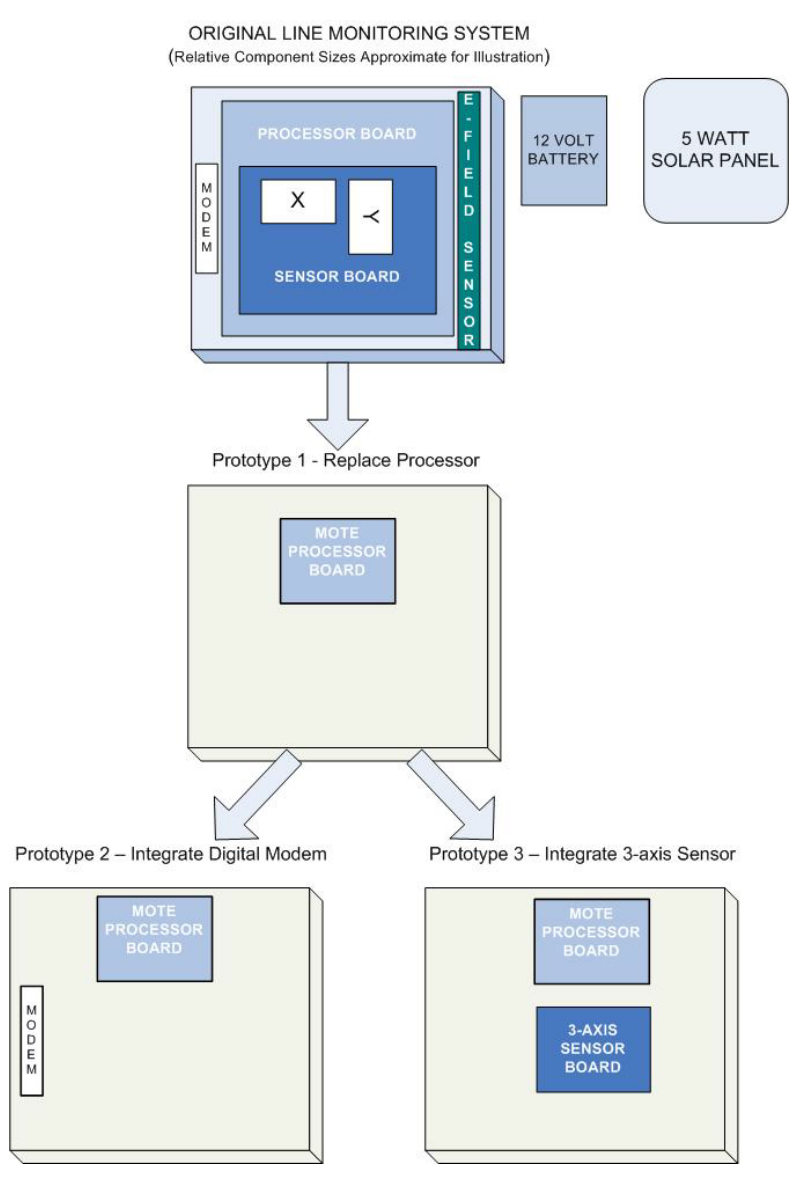

Figure 4-4: Development pathways corresponding to Phase 1 and Phase 2 prototypes. Items in color show the components being replaced and tested in each prototype stage. Prototype 1 has a replacement Mote processor, (Sensor, power, and communication modules remain the same as original). Prototype 2 adds a digital modem and integrates this with the Mote processor (Sensor and power modules remain the same as the reference system). Prototype 3 adds a 3 -axis magnetometer and associated interface electronics and integrates these with the Mote processor (Power and communication modules remain the same as the reference system).
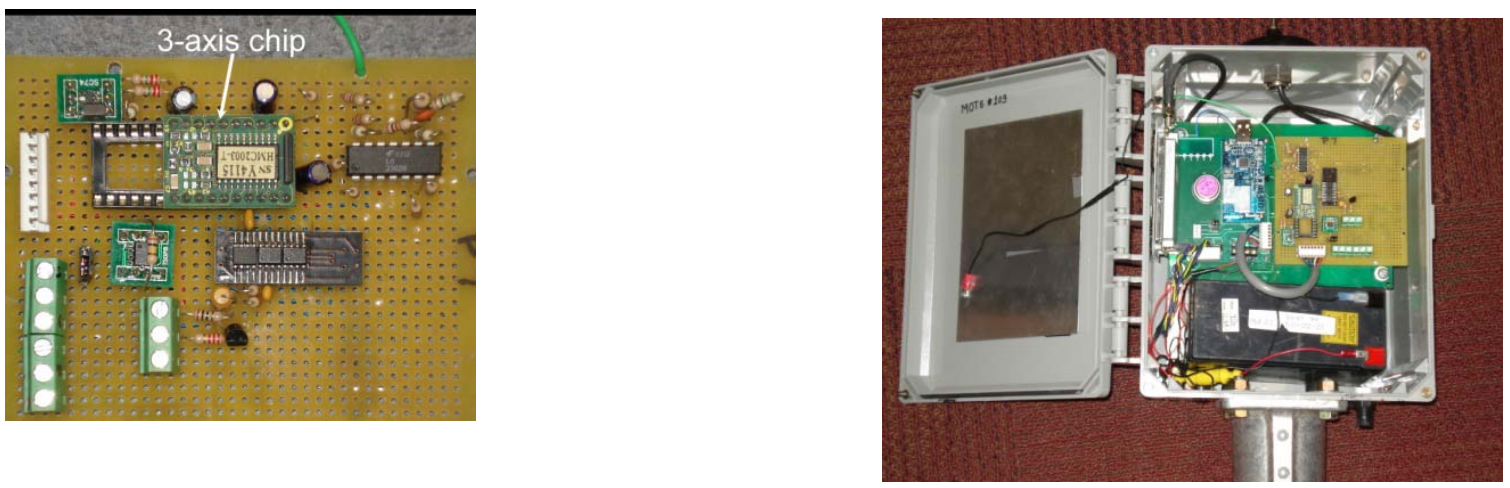
Figure 4-5: Prototype 3 showing the 3-axis magnetometer sensor board integrated into a monitor with a Mote processor.

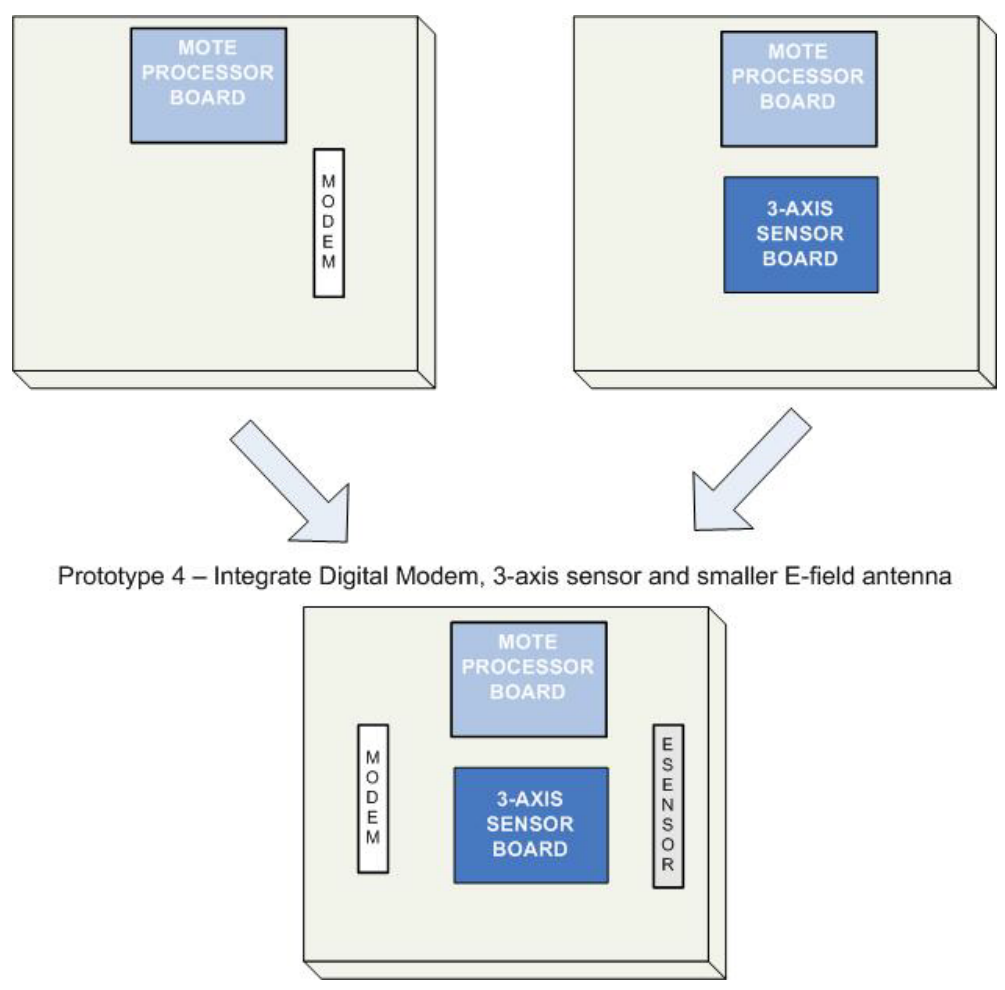

Figure 4-6: Prototype 4 development and integration 

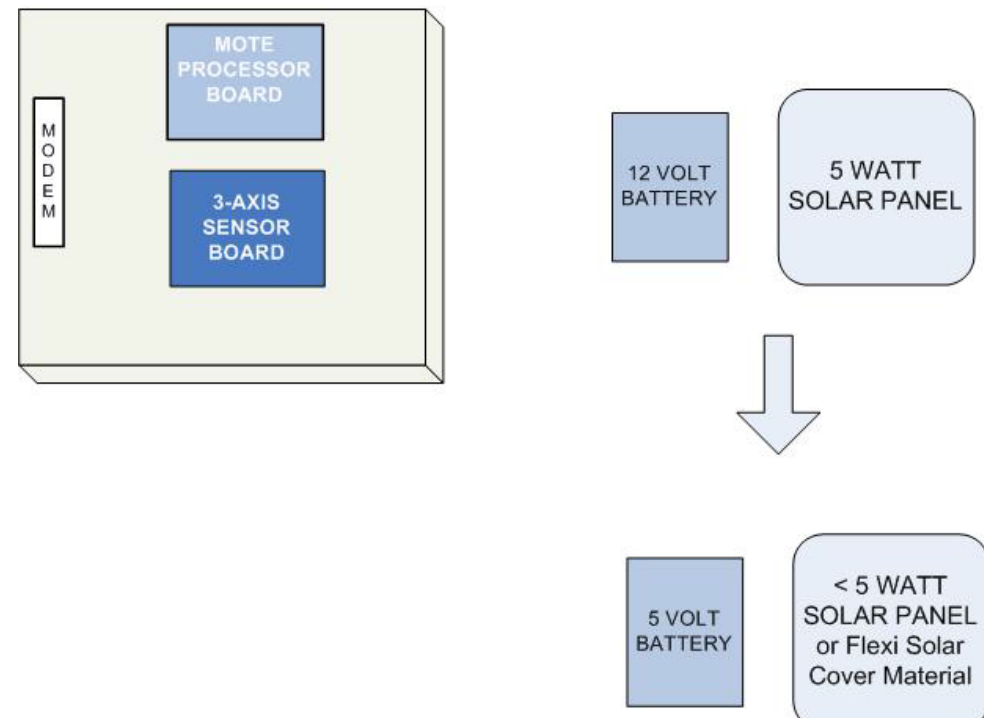

Figure 4-7: Prototype 5 development and integration

Prototype 5 contains all of the components of the new transmission line monitoring system developed under this Agreement with the US Department of Energy. Figure 4-8 shows a side by side comparison of the original transmission line monitoring system components in the original enclosure with the new transmission line monitoring system components in the original enclosure to compare size reduction. Processing (black outlined area), sensing (yellow outlined area) and power modules (blue outlined area) are highlighted in both photos to compare size. It should be noted that the original monitor components occupied the full depth of the enclosure also whereas the new monitor components are contained on a single board less than 1 inch in depth. 

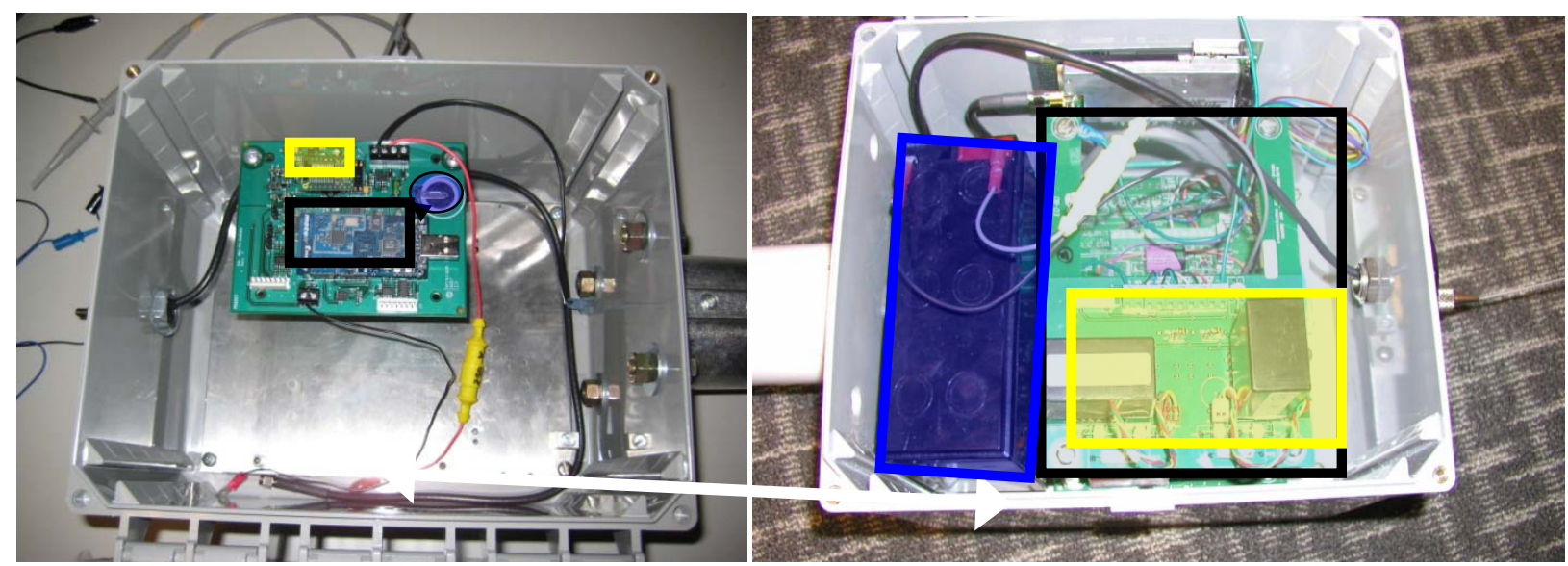

Figure 4-8: Side by side comparison of current integrated system board (left) to containing processor, 3axis magnetometer and power supply compared with original monitor (right) containing two separate processor and sensor boards and 12V battery.

\subsection{Enclosure Design}

The size of the existing commercial Genscape monitor is $21.5 \mathrm{~cm} * 26.5 \mathrm{~cm} * 17.2 \mathrm{~cm}$ (not including the $5 \mathrm{~W}$ solar panel). The proposed new component sizes were assessed and it is thought that the new components can be fitted inside an enclosure $12.7 \mathrm{~cm} * 12.7 \mathrm{~cm} * 7.6 \mathrm{~cm}$ (as shown in Figure 4.9 ).

\section{Component}

3-axis magnetic field sensor with interface circuit

Electric field sensor

Mote with interface board

Modem

Battery (lithium ion polymer battery)

\section{Size}

$9 \mathrm{~cm} \times 7 \mathrm{~cm} \times 3 \mathrm{~cm}$

$9 \mathrm{~cm} \times 3 \mathrm{~cm}$

$9 \mathrm{~cm} \times 7.5 \mathrm{~cm} \times 2 \mathrm{~cm}$

$6 \mathrm{~cm} \times 3 \mathrm{~cm} \times 1 \mathrm{~cm}$

$3.3 \mathrm{~cm} \times 4.8 \mathrm{~cm} \times 0.5 \mathrm{~cm}$ 


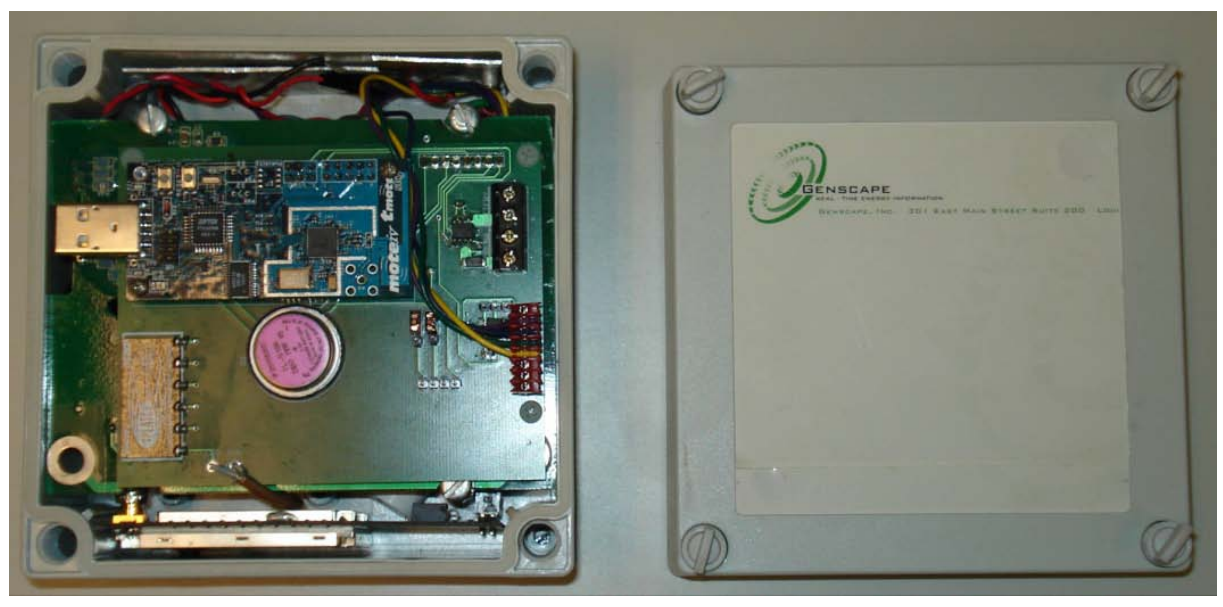

Figure 4-9: Form factor design for the new transmission line monitor

\section{Embedded Application Development}

Power line monitoring embedded applications were designed and developed for optimized data acquisition, on-board processing and data transmission protocols in TinyOS.

Embedded applications were developed in TinyOS to allow modem control and communication management for the new lower power CDMA modems. Firmware functionality includes

- Network connectivity and associated signal strength detection

- Data packet formatting

- Data packet transmission

- Schedule based transmission

- Exception based transmission

- Unit to packet reception central server communication

Protocols were developed to emulate the functionality of the current transmission line monitor firmware. A version of firmware in TinyOS was tested which interacts with the existing magnetic and electric field sensors to acquire basic magnetic and electric field data. Comparison of field readings acquired using the reference and prototype system under development in this project is shown in Figure 5.1. Embedded applications also allow modem control and communication management for the new lower power CDMA modems. 


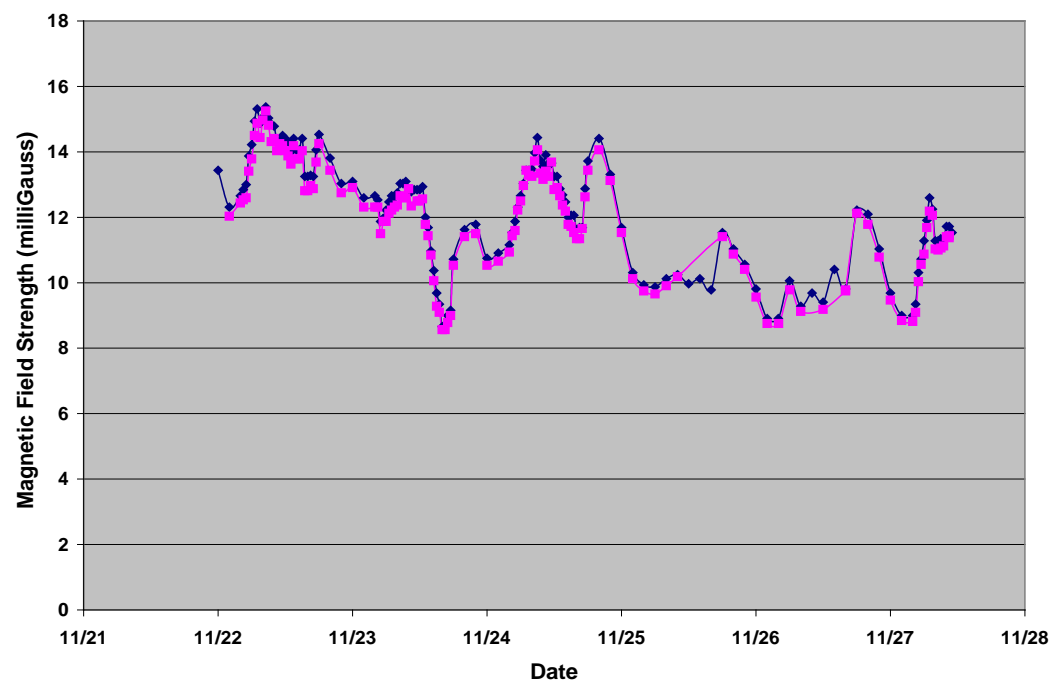

Figure 5-1: Magnetic field data acquired by the existing transmission line monitor (purple) and the prototype Mote-based transmission line monitor (blue) in a side-by-side deployment of both monitors at the transmission line test site shown in Figure 6.2.

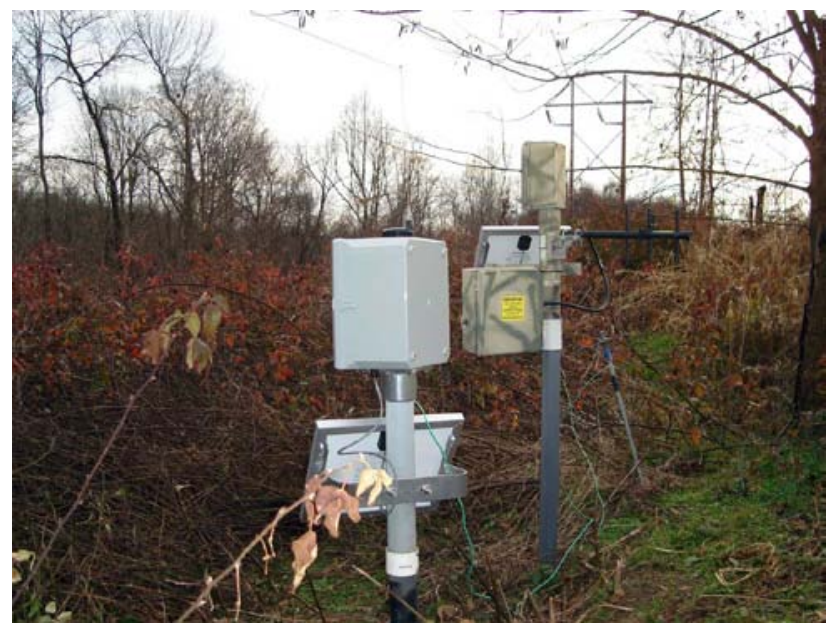

Figure 5-2: Side-by-side deployment of existing transmission line monitor (right) and a prototype Mote-based transmission line prototype (left) at a power line field test site. 


\section{Field Test and Deployment of Novel Sensors and Power Sources}

\subsection{AC Transmission Lines Network Deployment}

The transmission line monitors developed as part of this project were deployed at a number of heavily congested lines in the Mid-Atlantic area (defined by DOE in http://www.oe.energy.gov/nietc.htm, as shown in Figure 6.1). The lines monitored in this study carry critical flow to major metropolitan areas such as New York and Washington, D.C. These lines were chosen with reference to the August 2006 DOE National Electric Transmission Congestion Study ${ }^{15}$, and then sited for feasibility for monitoring by Genscape logistics staff. Additional heavily congested lines were also monitored in the West, Mid-West, ERCOT and Florida. The complete list is provided below:

Northeast

1. Massena to Marcy *

2. Branchburg to Ramapo*

West

3. Palo Verde Devers*

\section{$\underline{\text { ERCOT }}$}

4. Red Creek to Comanche Switch

Washington DC 500kV Loop

5. Louden-Doubs

\section{Florida}

6. Anclotte

Midwest

7. Forbes to Roseau*

* Mentioned in DOE 2006 Congestion Report

Prototype units were deployed side-by-side with the standard, existing commercial monitor. In general the mote prototype signal compares well with the standard calibration monitor (Figure 6.2. During the side-by-side deployment tests, data acquisition and data transmission rates and overall functionality for these units were high and compared well with standard monitors. In the case of the Massena-Marcy and Louden-Doubs lines, the mote prototypes detected and measured two independent outages on these lines as shown in Figure 6.3 and Figure 6.4. 


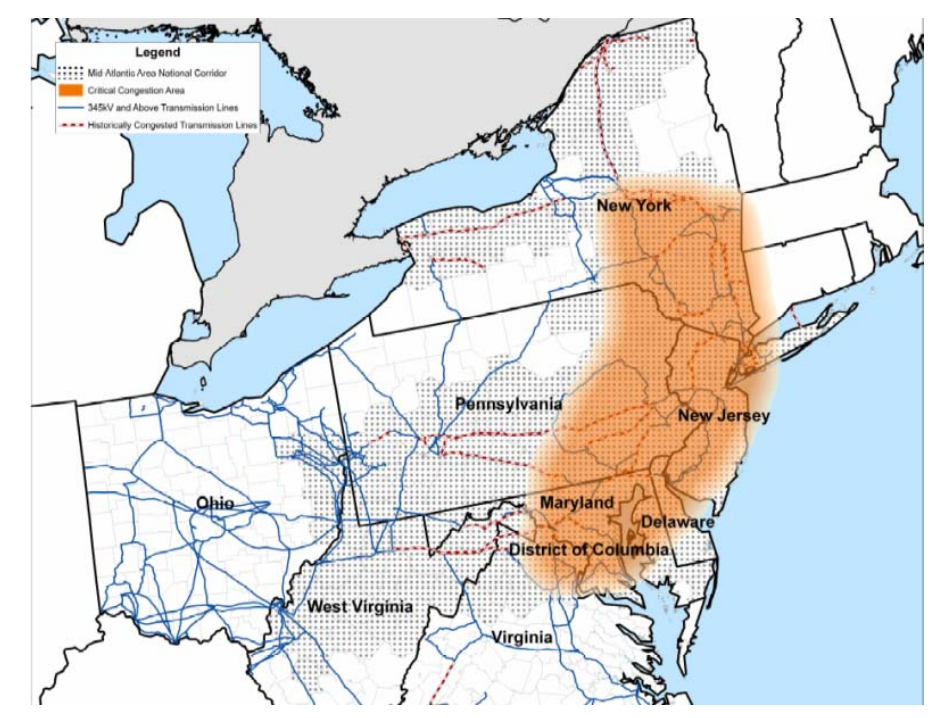

Figure 6-1: Mid-Atlantic Area Strategic Transmission Corridor

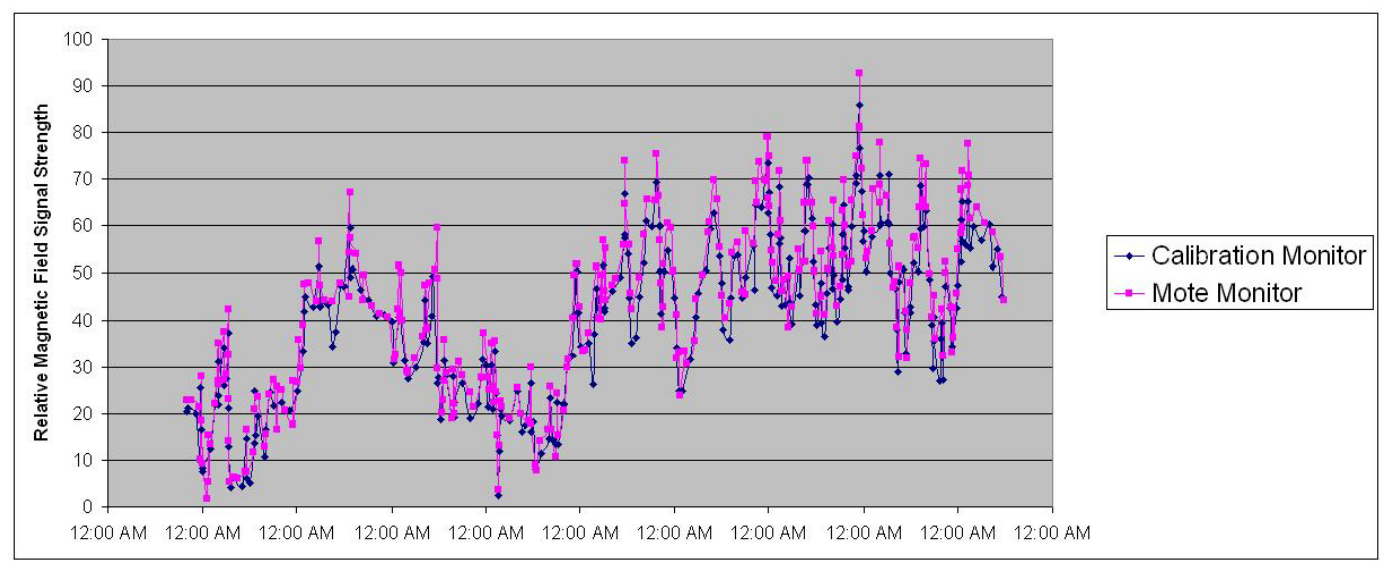

Figure 6-2: Mote prototype transmission line monitor compared with standard calibration monitor 


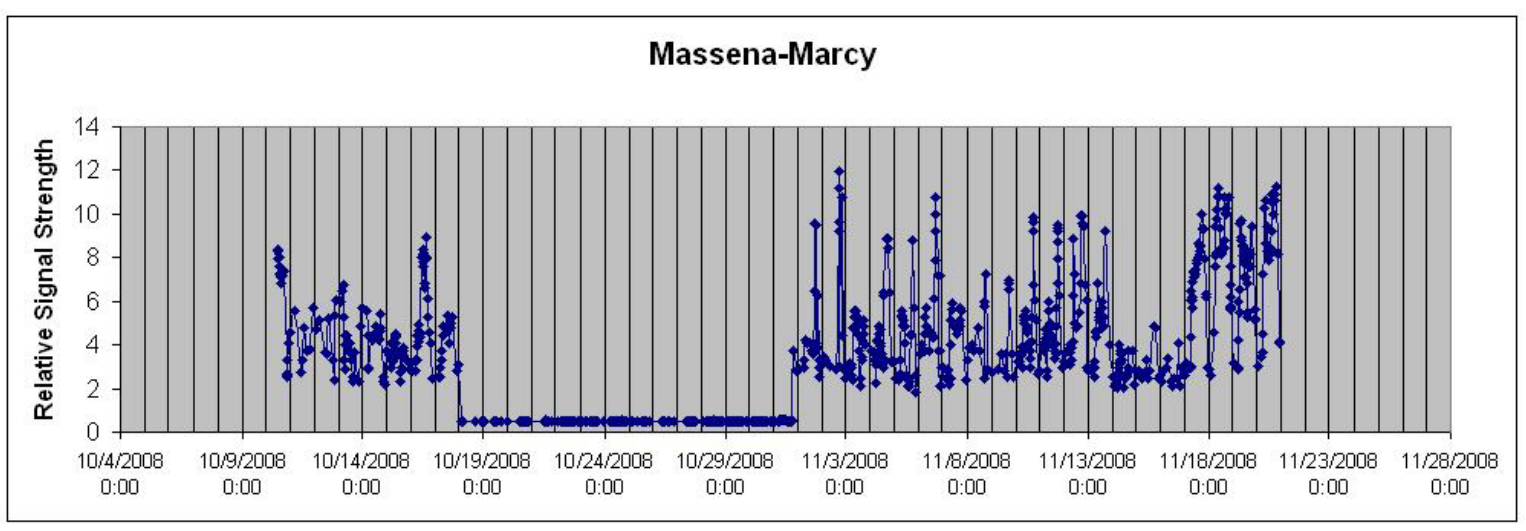

Figure 6-3: Transmission line data from the prototype monitors for Massena-Marcy line outage event, October 18, 2008.

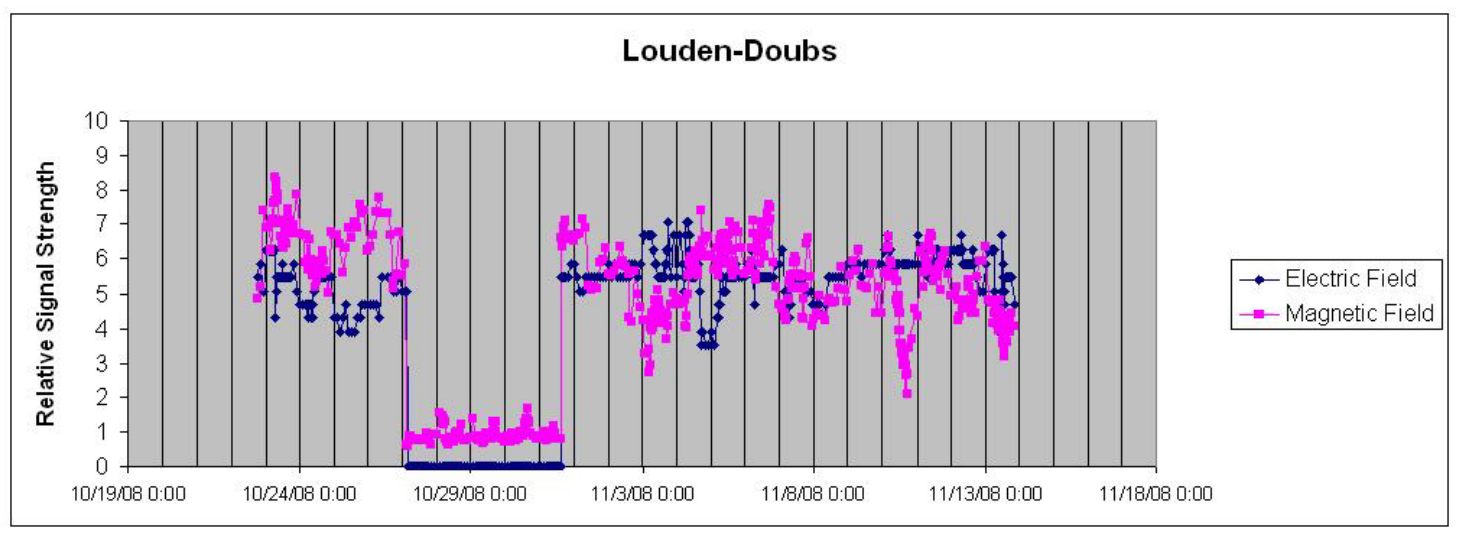

Figure 6-4: Transmission line data for Louden-Doubs line from the prototype monitor for line outage event, October 27, 2008.

\section{Server Side Application Development}

A custom application was developed to perform data reception, storage, data processing, alert processing, geographical processing (e.g., event clustering, monitoring by location), and data delivery and integration into the Oakridge National Laboratory (ORNL) VERDE platform (See Appendix A for a presentation of the integrated power line data as part of the VERDE application).

\subsection{Application Functionality Overview}

The transmission line event analysis and visualization application architecture developed during this project is outlined in Figure 7.1. The application components developed as part of this project are as follows; 
1. Implementation of a Google Earth based transmission line data viewing application. Google Earth is a low cost geographical information system (GIS) platform. This application developed here allows the presentation and visualization of transmission line data based on event analysis.

2. A Java-based desktop tool (http://openmap.bbn.com/) that allows transmission line data to be retrieved from a master database, analyzed for geographic and/or temporal clustering of data events and fed to Google Earth to be visualized based on the outcome of these analyses.

3. A custom routine to geocode imported transmission line data and power line monitor locations for use within the Google Earth application.

4. Analysis applications were developed to interact with the incoming data to deliver relevant alert and geographical based information direct to the visualization interface.

5. A password protected website was developed for third parties to serve as the portal for access to the transmission line data and alerts.

6. A Google Earth web service to allow remote access to the transmission line data via third party Google Earth applications such as VERDE at the third party site. 


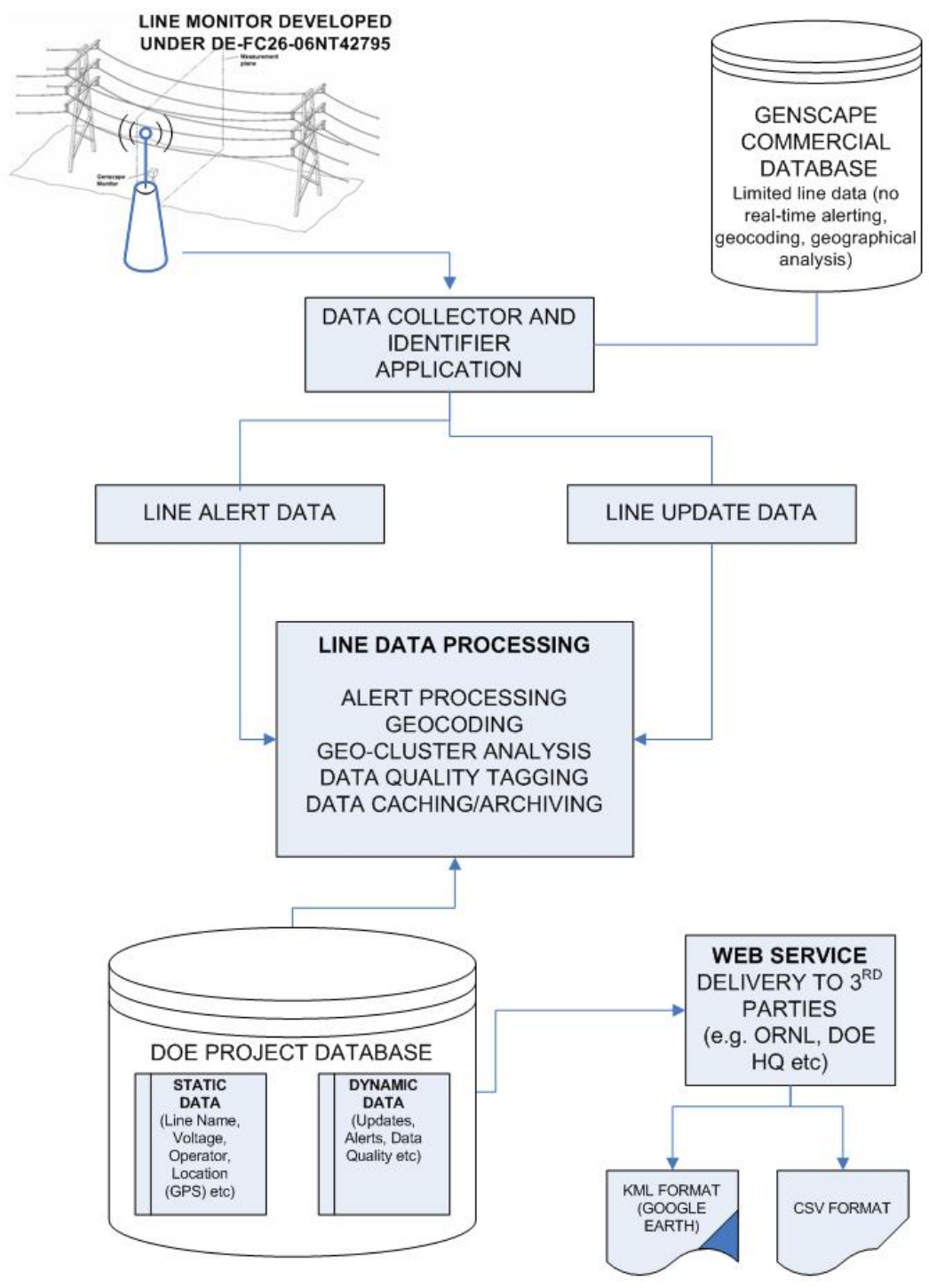

Figure 7-1: Application overview for processing of transmission line alerts and transmission of line alerts to third parties.

\subsection{Monitor and Transmission Line Visualization}

A geocoding routine was built to import transmission line data and power line monitor information (lat, long coordinates, line voltage, line capacity) into the Google Earth platform as a data layer (See Figure 7.2) from a master reference database. This information can be easily visualized in a pop-up window on mouse over of the power line monitor location in the Google Earth layer. In addition, a layer representing the transmission line network was loaded into the Google Earth application. In this way, the power line monitor location relative to the line it is monitoring can be easily visualized. 


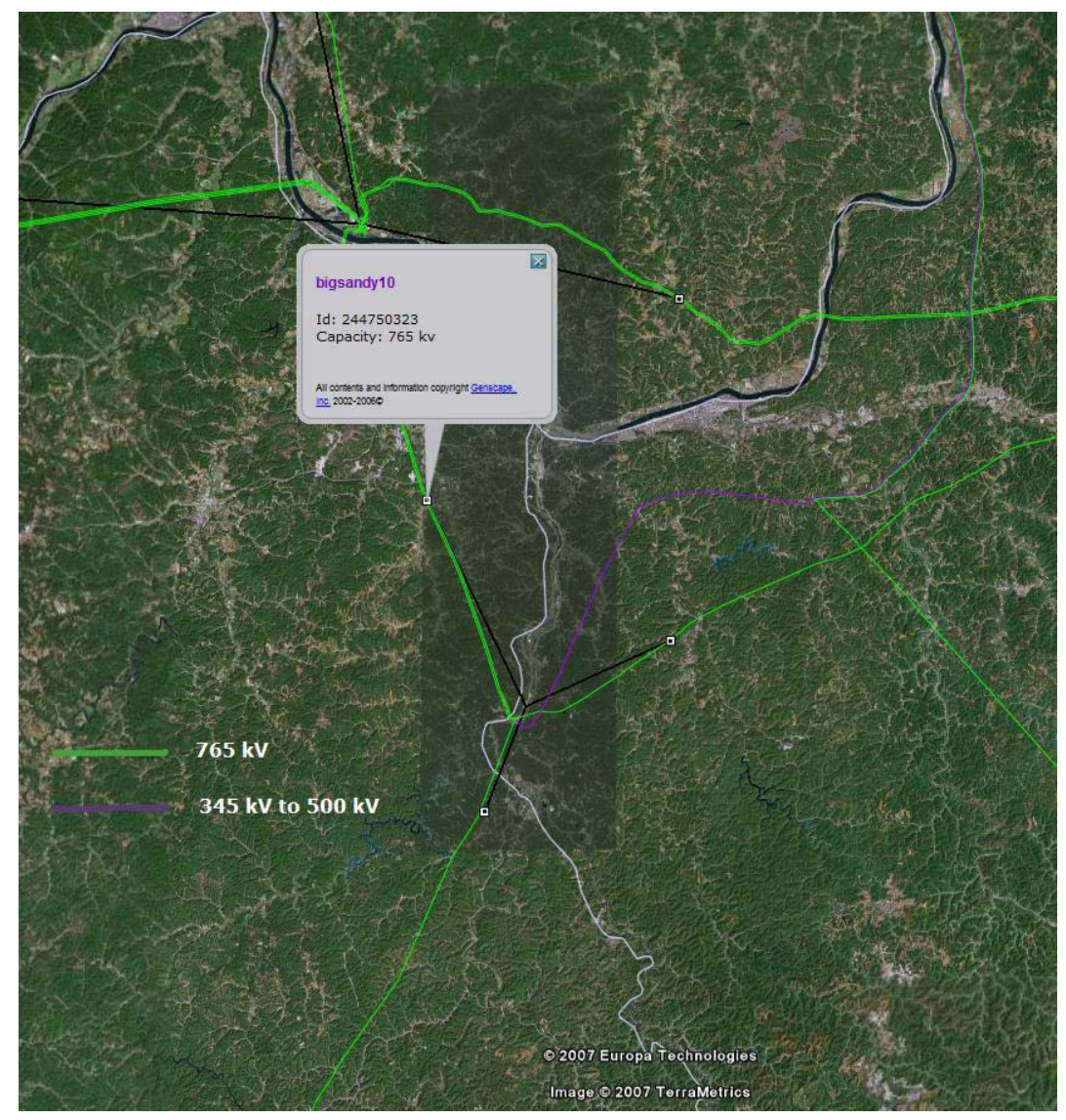

Figure 7-2: Google Earth image showing transmission line monitors (square icons) geolocated with transmission line data. Available line data (alerts, name, capacity voltage etc) can be presented through the Google Earth interface

\subsection{Transmission Line Alert and Alert Visualization Application}

In order to build effective event detection and alerting, a suite of transmission line message filters were developed. The power line monitor delivers exceptions calls based on a variety of magnetic and electric field changes at the line. Not all of these variations indicate major events such as line outages, but rather are associated with transient line changes, weather conditions, monitor malfunction and other reasons. For effective filtering of the data into true line outage information, the following was developed; 
- clustering analysis methods to detect synchronous exceptions within defined time periods (temporally clustered events)

- clustering analysis methods to detect co-located exceptions within defined geographic regions (geospatially clustered events)

- analysis methods based on association with a defined grid component such as a substation or a power plant or a particularly congested region of the grid.

An application was developed in Google Earth to filter transmission line data using the following four factors: severity (number of alerts); synchronization (number of alerts inside a defined time window); geospatial (number of alerts fired in a defined geographical area) and grid component association (e.g. lines associated with a specific power plant). The user of the application is able to define which filters to apply to incoming data.

Figure 7.3 and Figure 7.4 show example output from the geospatial filtering algorithms. The model case demonstrated is a significant Saskatchewan transmission line event (September 18, 2007) resulting in a power outage to $60 \%$ of the Saskatchewan province (http://www.cbc.ca/canada/saskatchewan/story/2007/09/18/power-outage.html\#skip300x250). The initial cause of the power loss in Canada was the loss of a large number of transmission lines due to storms in Minnesota and North Dakota. This event was simulated and used to develop alert mechanisms for the Google Earth application.

Filtering algorithms were optimized to visualize major transmission line outages during the event as distinct from insignificant background transmission line activity occurring simultaneously nationwide. 


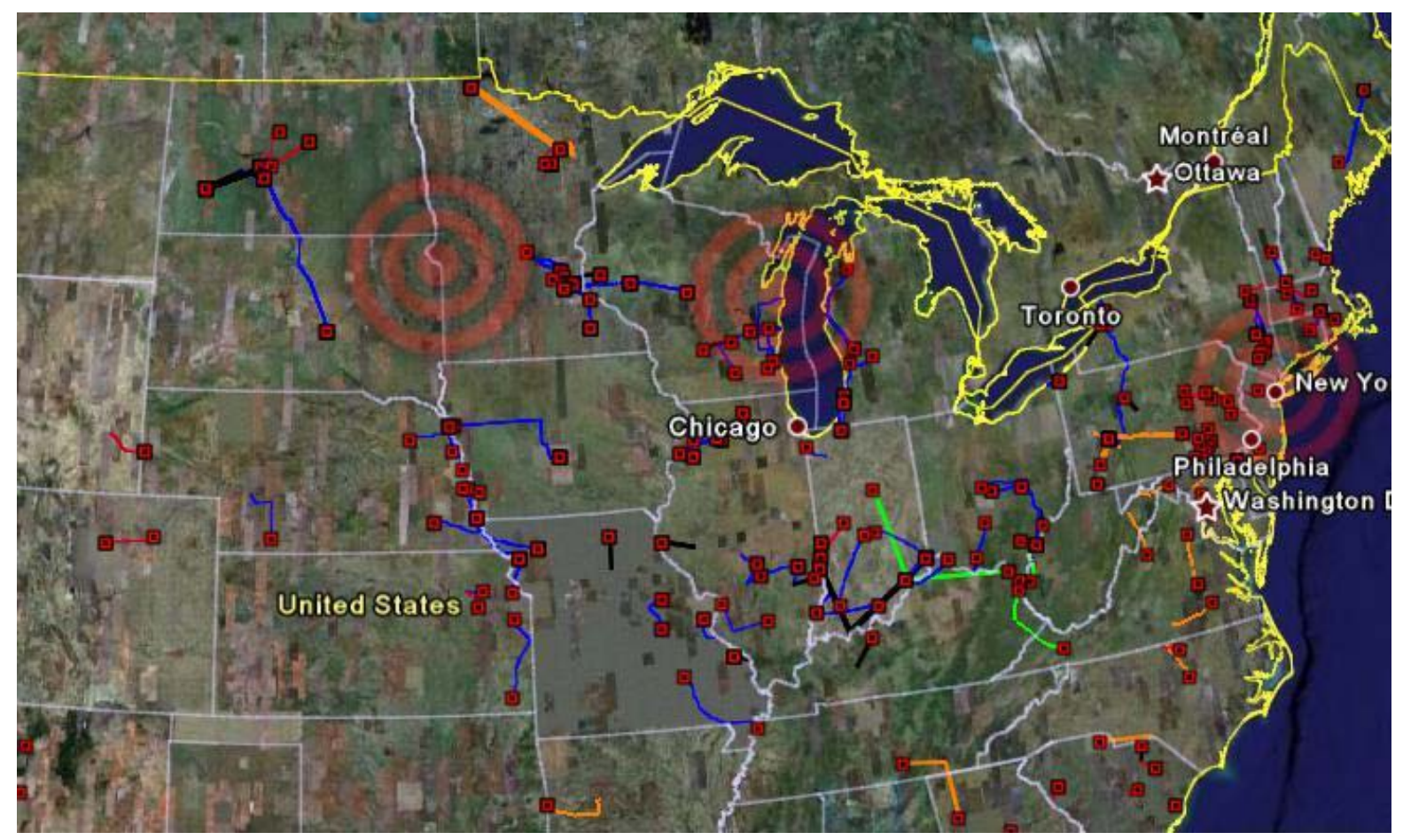

Figure 7-3: Data from a three hour window covering the September 18 event with no filtering applied.

Figure 7-3 shows data from a three hour window covering the September 18 event. Red squares denote all incoming transmission line alert messages from Genscape monitors. The red bullseye icons denote frequency event alerts from the Genscape frequency monitoring network (these are associated with outages of large generating units). The September 18 event involved the loss of a large number of transmission lines across Minnesota and Wisconsin west of Lake Michigan. Apart from the occurrence of two frequency events in that locality, the transmission line data in this format does not distinguish the event from other events picked up by the transmission line network from other areas such as the Ohio Valley and the Northeast at the same time. 


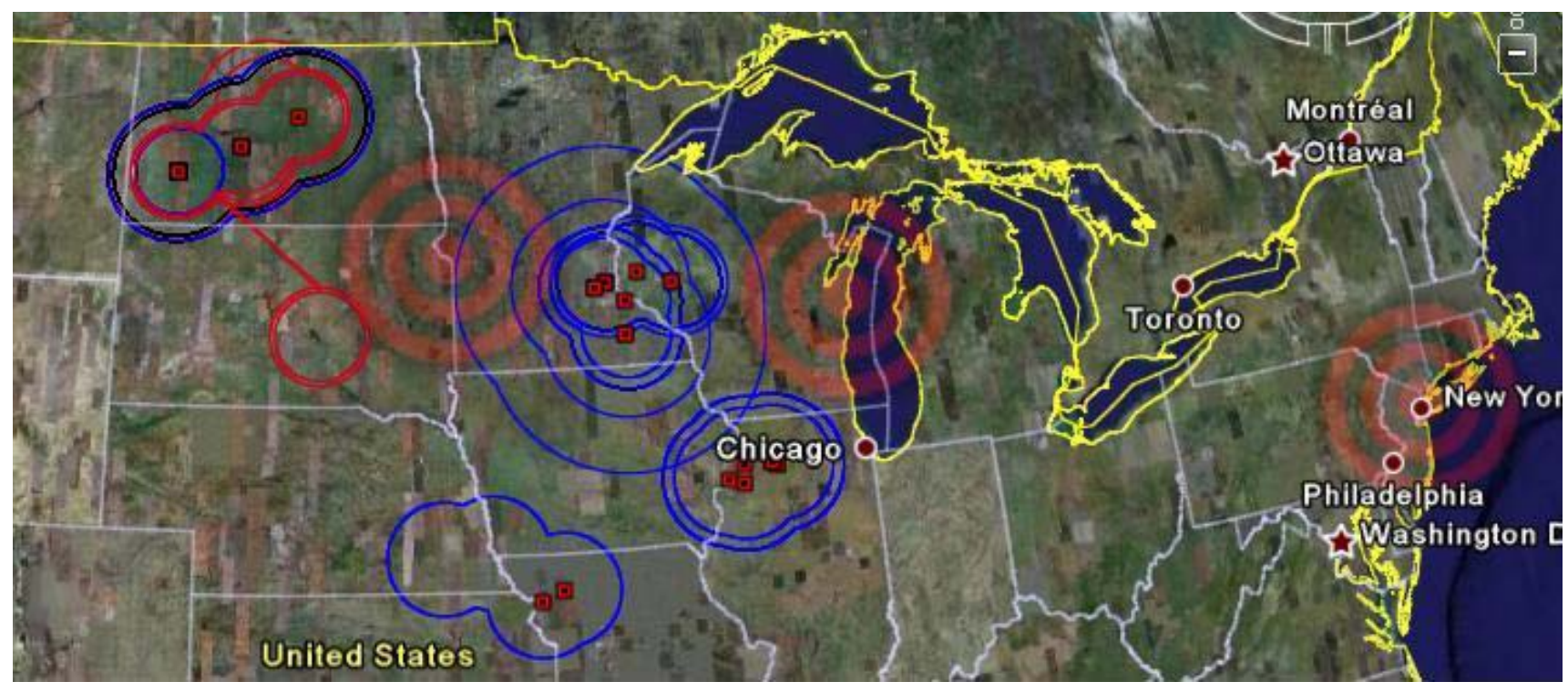

Figure 7-4: Post-filtered view of the September $18^{\text {th }}$ event showing a concentration of events passing the filter criteria in the Minnesota and Wisconsin areas.

A filter was developed to correlate transmission line alert messages which are geographically clustered, and coincident within a specified time window. This post-filtered view is shown in Figure 7.4, and clearly highlights the area involved in the September $18^{\text {th }}$ event. The number and diameter of the concentric circles shown are a function of the severity of the event. The color of the concentric circles denotes the line voltages involved. Although one frequency event is located in the New York area, the majority of other Ohio Valley and Northeast transmission line events are not sufficiently associated in time or geospatial location to pass the filter and are no longer seen as significant.

Figure 7.5 illustrates an alert visualization associated with lightening storms associated with a tornado along the North Carolina and South Carolina border region. Red lines indicate transmission lines which have simultaneously transmitted exception messages to the data center. The red shaded alert area is the result of filtering based on synchronicity and geographical clustering of incoming transmission line data. The user can access this view via an alert panel in the Google Earth application. The US national map (which is the default view) is zoomed into this region. The program also allows weather overlays associated with the region for the same timeframe to be imported into the application for viewing. This can be useful in ascertaining the reason for the alerts. The lightening strikes in this case resulted in the loss of a power plant on the coast (seen as the cluster of red lines emanating from a single point representing the power plant). 


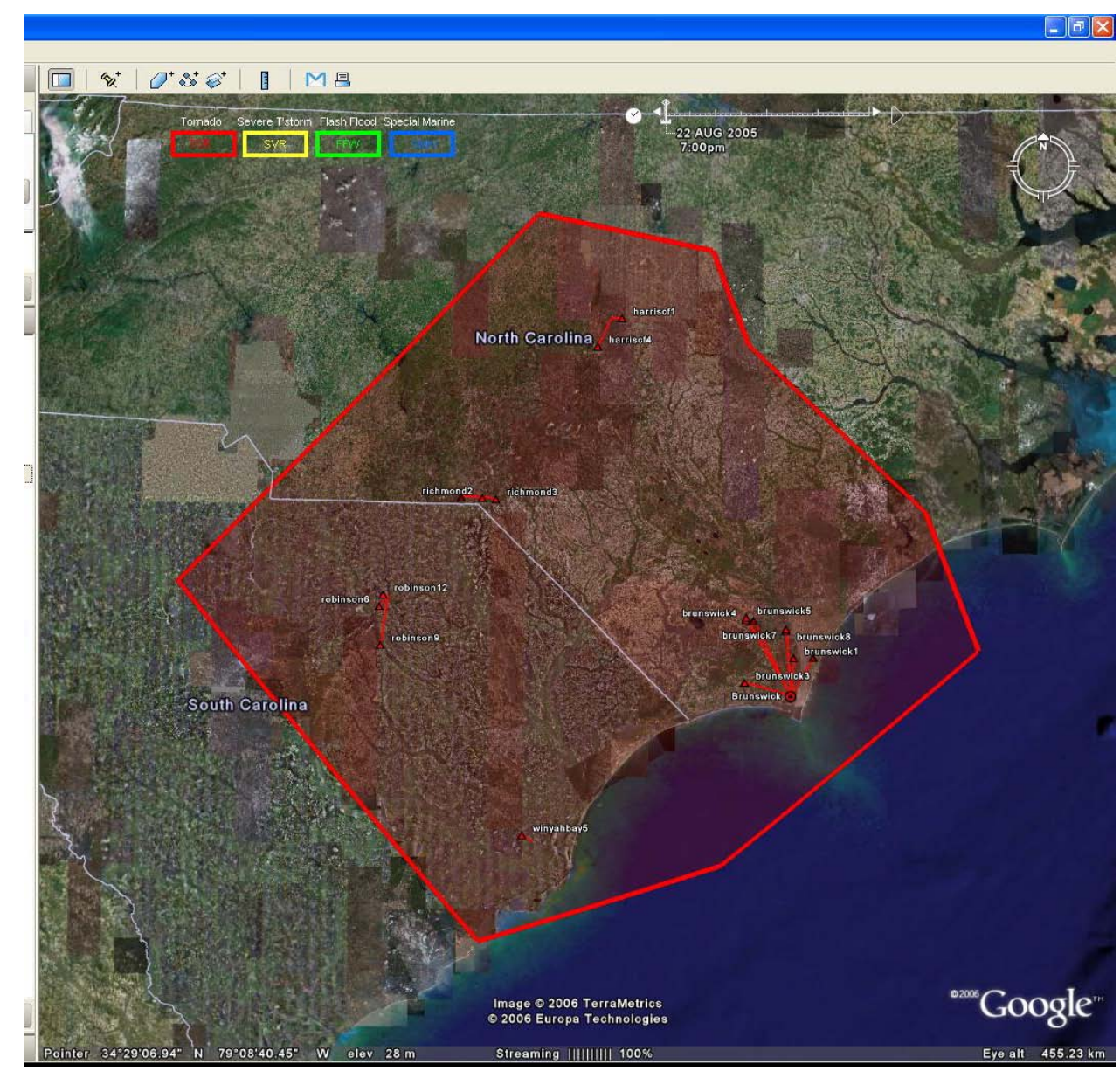

Figure 7-5: Google Earth visualization of transmission line events (red lines) associated with a lightening strike during tornado activity in the region shown.

\section{Data Viability and Integration}

\subsection{Data Delivery to $3^{\text {rd }}$ parties and Data Integration}

A near real-time Google Earth file $(\mathrm{kml})$ internet server was launched and released to Oakridge National Laboratory in March 2008. The site www.doe.genscape.com is password protected and allows the user access to transmission line data reports, alerts. The main menu screen is shown in Figure 8.1. Historical Data can be loaded using the Parameter Input Date Range Window. The Genscape Transmission Lines and Plants link connects to a KML file containing US transmission lines and power plants. This file is meant as a user reference when viewing alerts. 
Below this link are sample events of significance. These sample files show how the alert applications works in the cases of actual transmission line outage events (NERC TLR Level 5 Event, Manitoba Hydro Flowgate 6002 September 18, 2007 and BlackOak - Beddington event Dec 20, 2007). The Activity over the previous 24 hours link provides a kml file of most recent transmission line activity. Data includes all messages received from monitored transmission lines, specific alerting to events where a number of transmission substations are involved simultaneously within a pre-defined period of time.

Other kml files can be delivered by the application, such as a file generating alerts for significant (where the level of significance is a user defined parameter) changes in line flow (MW) within a user defined timeframe.

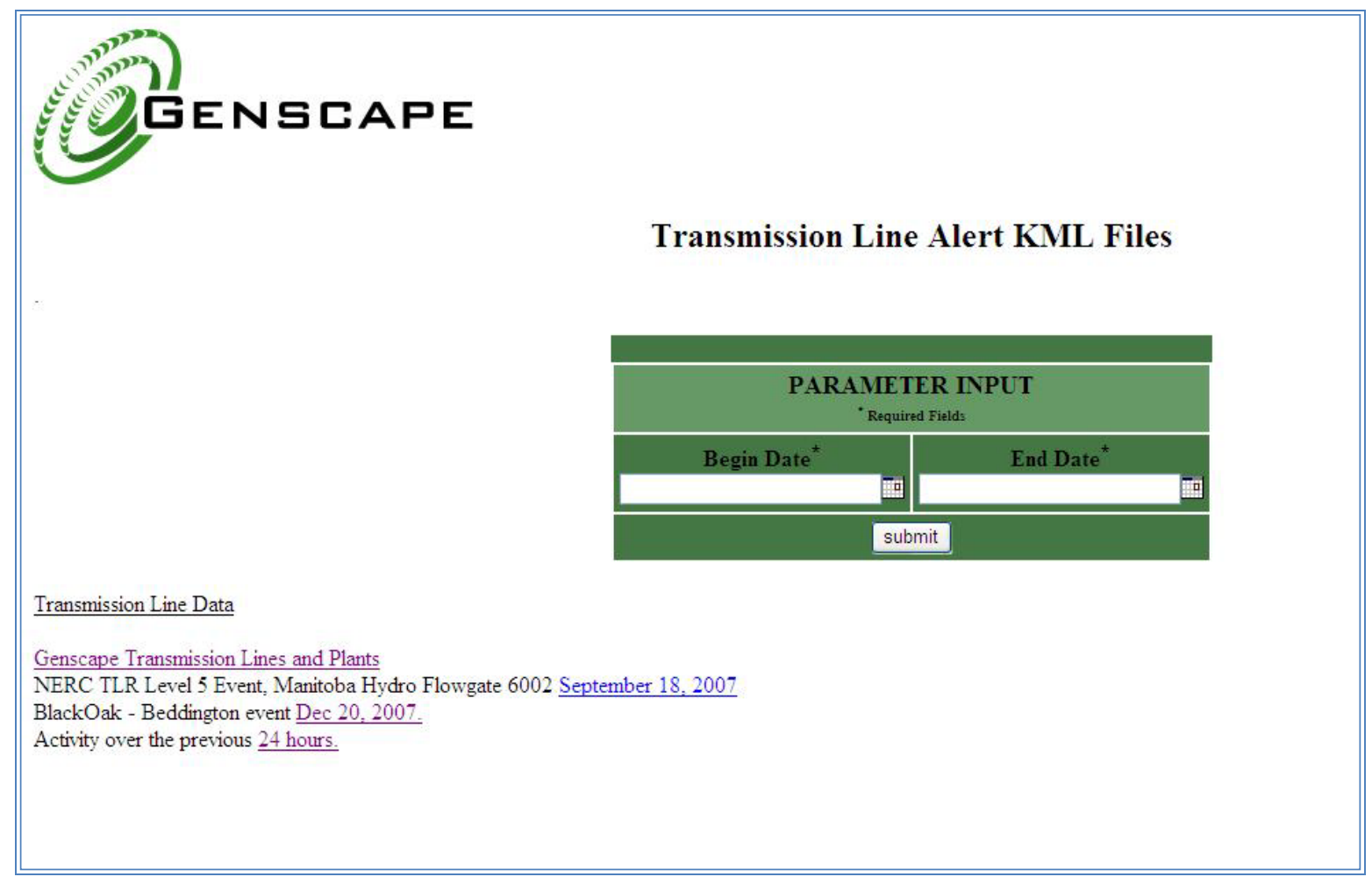

Figure 8-1: Main menu screen on transmission line kml file server application.

Two additional data streams were added to the Google Earth interface are a layer of real-time data highlighting transmission lines that have shown substantial change in flow, defined as +/$25 \%$ relative to their nominal capacity, and real-time flow charts for the lines where substantial changes in flows have occurred. 


\section{Frequency Alerts for Transmission Line Outages}

Frequency monitoring was explored as a complementary monitoring technology to detect transmission line outages. The loss of a transmission line can be detected with a grid frequency monitor as a distinct oscillatory signal. Genscape has deployed over 85 of these monitors on the United States power grid to detect generation and transmission line loss events in real-time. The coincidence of transmission line alerts with the occurrence of significant frequency disturbances characteristic of major transmission line outages have been explored here using the Google Earth application. A typical transmission line outage event captured by three frequency monitors was recorded for the loss of a high voltage transmission line in the Eastern Interconnect on February 1, 2007, as shown in Figure 8.2. Algorithms have been written to capture information from the frequency monitoring network and overlay this information onto the Google Earth transmission line visualization application (as shown in Figure 8.3).

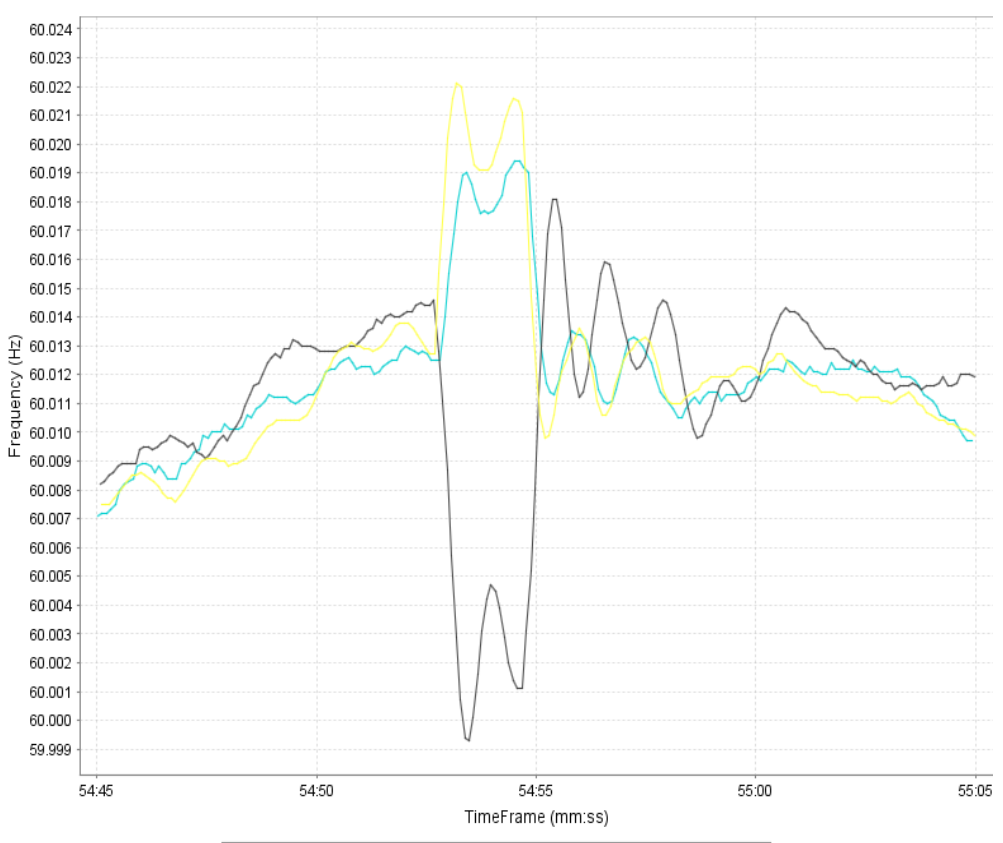

Figure 8-2: Transmission line outage signal detected using grid frequency monitoring network 


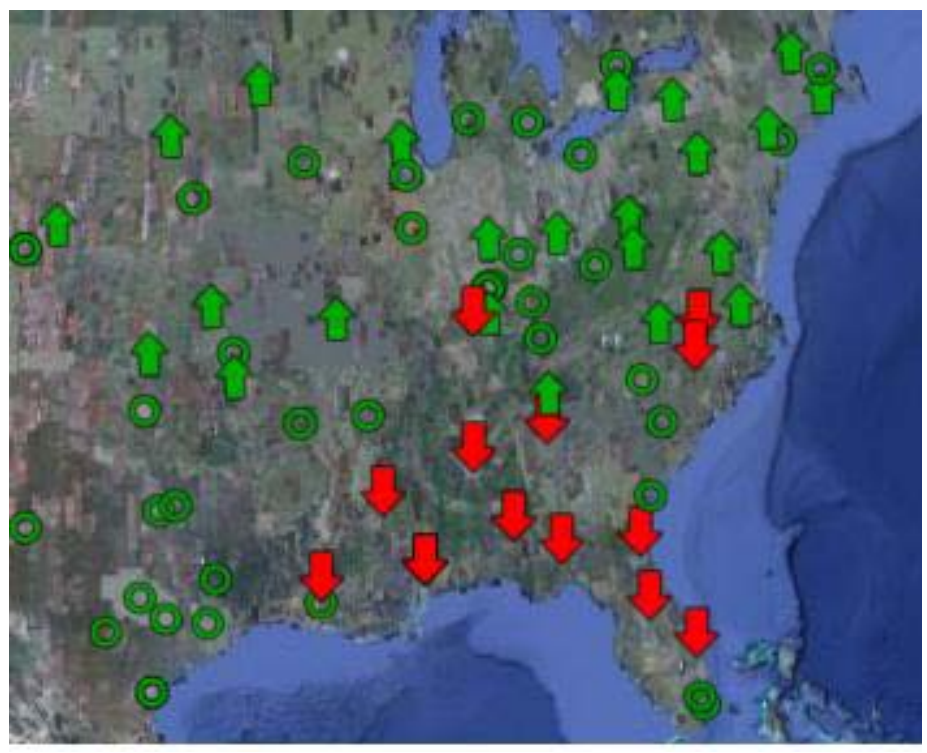

Figure 8-3: Frequency information from a transmission line outage event displayed on the Google Earth application. Red arrows show units detecting under-frequency signals. Green arrows show units detecting over-frequency signals.

Current practices and protocols were identified in the electric utility industry with the assistance of E. On US (Kentucky Utilities), to identify how power line outages and data are currently handled by Supervisory Control and Data Acquisition (SCADA) systems and Energy Management Systems (EMS) in local power distribution control centers in Kentucky. ORNL were provided with the Genscape Power North America 2.4 data product for 12 months in order to gain experience working with this data in order to determine how a source of $3^{\text {rd }}$ party realtime power line data may complement other grid information that ORNL is already providing to DOE/OE Headquarters and other $3^{\text {rd }}$ party data available to DOE/OE Headquarters for grid reliability needs.

\section{Cost Analysis for Wireless Transmission Line Networks}

In order to consider the use of independent wireless power line monitoring networks to relay real-time information on the US grid, the associated costs of an network are classified as follows;

\section{Transmission Line Monitor Hardware Costs}

1. Sensing devices and associated processor components

2. Power sources to enable wireless operation (e.g. batteries, solar panels) 


\section{Cellular communication modems}

The total cost of basic components used in the line monitor developed in this project is approximately $\$ 500$. The distribution of hardware costs for an individual transmission line monitor is shown in Figure 9.1

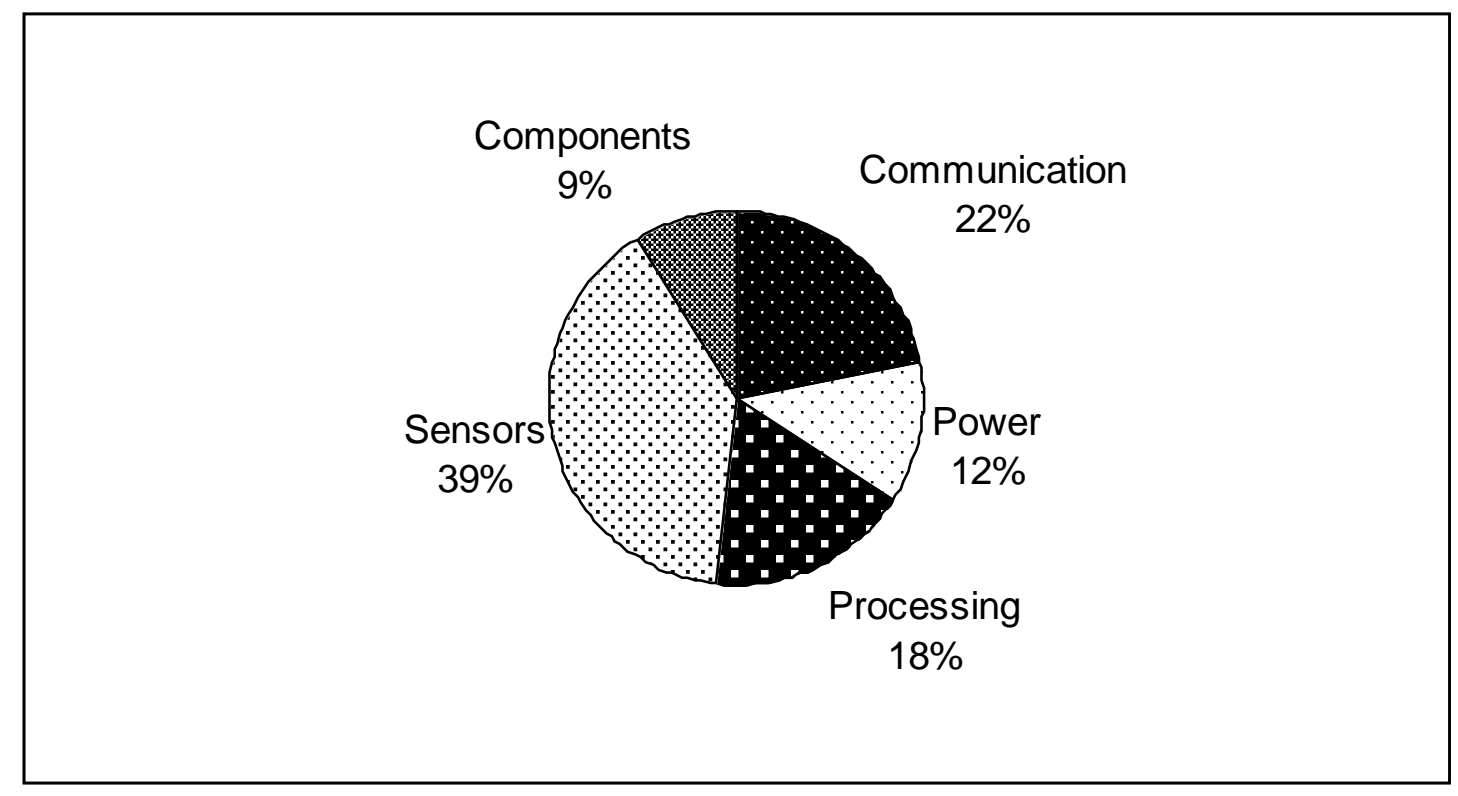

Figure 9-1 Distribution of costs associated with internal components of the wireless electric transmission line monitor.

\section{Network deployment costs}

1. Field installation

2. Easement fees

The cost of deployment varies greatly depending on the logistics of installation, installation teams concentrated in single areas can greatly reduce time and expenses in deploying field monitors.

\section{Ongoing operation and maintenance costs}

\section{Cellular communication costs}

Since a typical wireless electric transmission line monitor sends only small packets of data back to a central data server, recurring communication costs associated with cellular communication are typically less than those encountered by normal cell phones accounts. The cost per monitor is approximately in the range $\$ 10$-\$20 per month. 
2. Maintenance costs

These costs depend on the system deployed and the degree of redundancy built into the system.

\section{REFERENCES}

1. Final Report on the August 14th Blackout in the United States and Canada https://reports.energy.gov/

2. Genscape powerline monitoring technologies http:/www.genscape.com, US Patents 6,714,000 6,956,364 7,088,090 7,398,168 7,571,028

3. Mote: http://computer.howstuffworks.com/mote1.htm

4. Mote commercial development companies: Crossbow http://www.xbow.com/, Dust Networks http://www.dustnetworks.com/

5. http://www.ornl.gov/sci/electricdelivery/vis_VERDE.html

6. "Energy Scavenging for Wireless Sensor Networks with Special Focus on Vibrations” Roundy, Wright, Rabaey 2004 (Kluwer Academic)

7. Roundy, S. 2004. “A piezoelectric vibration based generator for wireless electronics," Smart Mater. Struct. 131131

8. Lin, J.T.; Lee, B.; and Alphenaar, BW,, "Magnetic coupling of piezoelectric cantilever for enhanced energy scavenging' Submitted Smart Mater. Struct.

9. Lin, J.T, and Alphenaar, BW, "Enhancement of energy harvested from a random vibration source by magnetic coupling of a piezoelectric cantilever,” In Press, Journal of Intelligent Material Systems and Structures.

10. Electric Cables IEEE Transactions on Instrumentation and Measurement, Vol. 45, No. 2, April 1996 Alexandler V. Mamishev, Shayne X. Short, Ta-Wei Kao, B. Don Russell.

11. Roctest/Telemac www.roctest.com 
12. Three-Axis Magnetic Sensor Hybrid. Honeywell. Solid State Electronic Center. 1-4. $<$ www.magneticsensors.com>

13. Bartington Instruments 21 July $2008<$ http://www.bartington.com/index.php?id=19>

14. Jones, Walter. MicroMag3Testing. Genscape Inc. Louisville, 2007. 1-15

15. National Electric Transmission Congestion Study, August 2006 http://www.oe.energy.gov/Congestion_Study_2006-9MB.pdf 


\section{Appendix A}

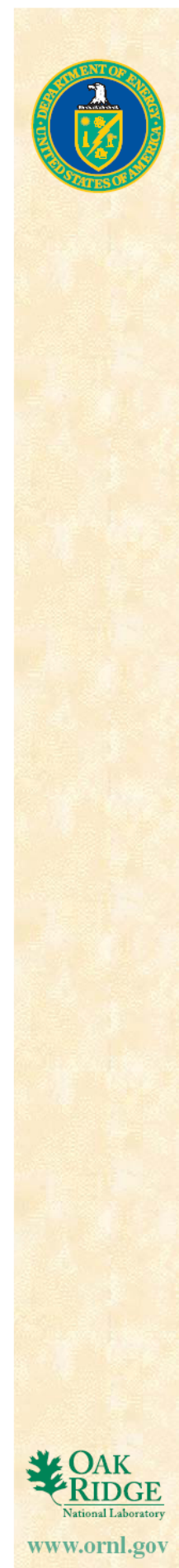

\section{Oak Ridgenational Laboratory Fact SheET \\ MANAGED BY UT-BATTELLE FOR THE DEPARTMENT OF ENERGY}

\section{Visualizing Energy Resources Dynamically on Earth VERDE: A Real-time National Visualization Resource}

\section{Situation Analysis}

Major power outages in the United States over the past decade have a recurring theme - the lack of wide-area situational understanding was a key factor that contributed to blackouts and also in managing the preparedness for and response to destructive events. Real-time geo-visualization capability characterizes the dynamic behavior of the electric grid across multi-regions, substantially mitigating the risk of and accelerating the recovery from a large area power disruption.

\section{Technology Pathway}

Oak Ridge National Laboratory (ORNL) is developing a national visualization capability for the U.S. Department of Energy's Office of Electricity Delivery \& Energy Reliability. This resource will enable real-time status of the electric grid and critical energy sectors and eventually a "health status" of the nation's electric infrastructure to assist federal agencies in the coordination and response during major events such as wide-area power outages, natural disasters and other catastrophic events. The following functions will be featured in VERDE:

- Real-time status of the electric grid

- Energy infrastructure integration

- Real-time weather \& other data streams

- Grid behavior modeling and simulation

- Extreme contingency analysis

\section{Partnerships}

ORNL is partnering with industry and universities to develop this strategic tool. Tennessee Valley Authority and other major utilities spanning multiple regions across the electric grid interconnection are providing real-time status of their system. Innovative businesses such as Genscape are providing additional datasets that will contribute to visualization and advanced modeling analyses.

Point of Contact:

Thomas King

Oak Ridge National Laboratory

P.O. Box 2008

Oak Ridge, TN 37831-6195

Phone: 865-241-5756

kingtjjr@ornl.gov
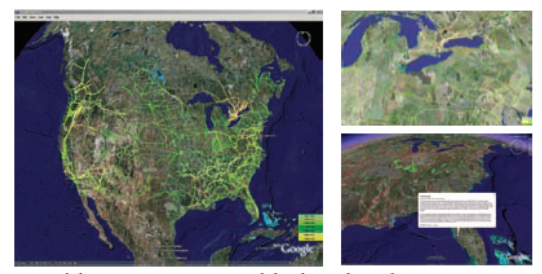

Wide-Area Power Grid Situational Awareness
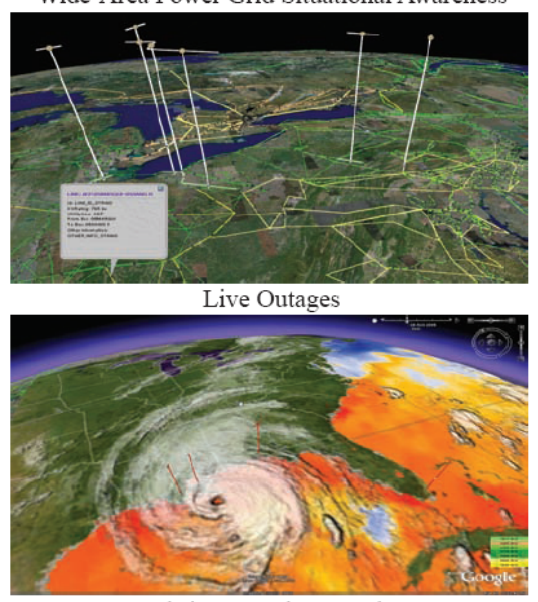

Real-time Weather Overlays

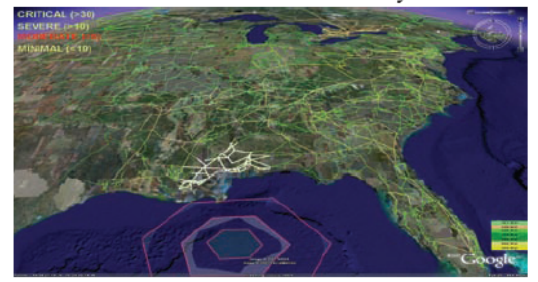

Impact Models Display and Animated Replay
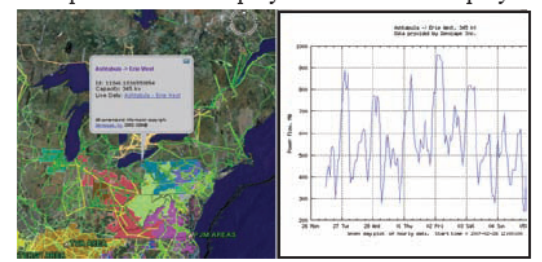

Streaming Analysis

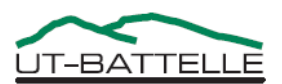

